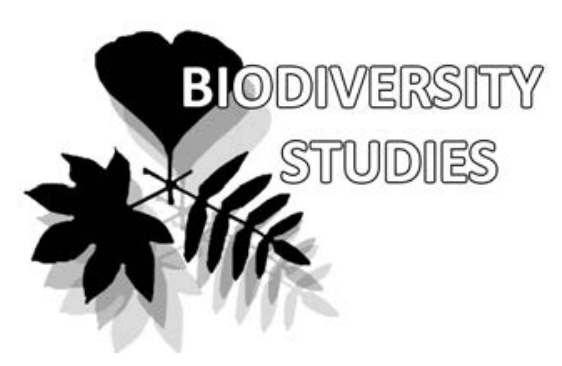

\section{Mosses of the southern Russian Far East, an annotated check-list}

\author{
Valentina Ya. Cherdantseva ${ }^{\dagger}$, Olga Yu. Pisarenko'*, \\ Michael S. Ignatov 2,3, Elena A. Ignatova ${ }^{2}$, Vladimir E. Fedosov², \\ Sergey V. Dudov ${ }^{2}$, Vadim A. Bakalin ${ }^{4}$
}

\author{
Valentina Ya. Cherdantseva ${ }^{\dagger}$ \\ Olga Yu. Pisarenko ${ }^{1 *}$ \\ e-mail: o_pisarenko@mail.ru \\ Michael S. Ignatov 2,3 \\ e-mail: misha_ignatov@list.ru \\ Elena A. Ignatova ${ }^{2}$ \\ e-mail: @.ru \\ Vladimir E. Fedosov ${ }^{2}$ \\ e-mail: fedosov_v@mail.ru \\ Sergey V. Dudov² \\ e-mail: serg.dudov@gmail.com \\ Vadim A. Bakalin ${ }^{4}$ \\ e-mail: vabakalin@gmail.com \\ ${ }^{1}$ Central Siberian Botanical Garden, SB \\ RAS, Novosibirsk 630090 Russia \\ ${ }^{2}$ Lomonosov Moscow State University, \\ Faculty of Biology, Geobotany Dept., \\ Moscow 119234 Russia \\ ${ }^{3}$ Tsitsin Main Botanical Garden RAS, \\ Moscow 127276 Russia \\ ${ }^{4}$ Botanical Garden-Institute FEB RAS, \\ Vladivostok 690024 Russia \\ $\dagger$ deceased \\ * corresponding author
}

Manuscript received: 01.10.2018

Review completed: 20.10 .2018

Accepted for publication: 22.10.2018

Published online: 24.10 .2018

\begin{abstract}
A B S T R A C T
The check-list of mosses of the southern part of the Russian Far East includes 816 species and 10 infraspecific taxa with references on their distribution in seven floristic regions within Primorsky and Khabarovsky Territories, Amurskaya and Sakhlinskaya Provinces and Evreiskaya Autonomous District. Seventy one species are excluded in the course of the check-list compilation, and 59 are commented as doubtful and erroneously reported from some of the Far Eastern regions, while 8 of them doubtful for the southern part of Russian Far East in general.
\end{abstract}

K e y w o r d s : bryophyte, flora, biodiversity, taxonomy, synonymy, Primorsky Territory, Khabarovsky Territory, Amurskaya Province, Sakhlinskaya Province, Evreiskaya Autonomous District

\section{P E 3 Ю M E}

Черданцева В.Я., Писаренко О.Ю., Игнатов М.С., Игнатова Е.А., ФеАосов В.Э., Аудов С.В., Бакалин В.А. Мхи южной части российского Аальнего Востока: аннотированный список. Чеклист мхов юга Российского Аальнего Востока вкАючает 816 видов и 10 внутривидовых таксонов со ссылками на опубликованные данные, иногда гербарные материалы по семи флористическим районам в пределах Приморского и Хабаровского краев, Амурской и СахаАинской областей и Еврейской автономной области. 71 виА искАючен из флоры мхов этого региона. А^я 59 видов обсужАаются сомнительные и ошибочные указания Аля некоторых районов, Аля которых они приводились, из них произрастание 8 видов в пределах рассматриваемой территории в целом сомнительно или не подтверждено образцами.

КАючевые слова: мхи, флора, биоразнообразие, таксономия, Приморский край, Хабаровский край, Амурская область, Сахалинская область, Еврейская автономная область

Content: Introduction ..................................................... 53

Check-list .......................................................... 54

Comments on species doubtful and erroneously reported from at least some regions ........... 70 List of excluded taxa ......................................... 72 Synonyms ............................................................... 74

\section{INTRODUCTION}

Present paper is addressed to the southern part of the Russian Far East, the region within the Primorsky and Khabarovsky Territories, Amurskaya and Sakhlinskaya Provinces and Evreiskaya Autonomous District (Fig. 1). It covers extensive area over $1500000 \mathrm{~km}^{2}$.

The southern part of the Russian Far East never had shield glaciations, including the coldest intervals of in the Late Pleistocene, although local glaciation at the highest elevations occurred at places. This fact makes the study area to be the largest among North Asian regions with boreotemperate mesophytic floras survived in situ ice age of Pleistocene. Other important characteristics of the territory include great variation in climatic variables, especially temperature and precipitation, providing changes from oceanic climate in Sakhalin and Kuril Islands to continental one in the western part of Amurskaya Province, where Dahurian steppe vegetation occurs. Mountains of the region are very diverse in chemical composition of both ancient bedrocks and more recent sediments, so both basic, acidic and neutral substrates are widely distributed.

The territory represents a transition between East Asian and Circumboreal floristic provinces, and it obviously comprises migration routes of boreal and temperate taxa followed the climatic fluctuations in the Pleistocene and Holocene. The predominance of north-south migrations corresponds to the sub-meridional orientation of mountain ranges. In addition, important routes occurred along the land bridges united Sakhalin and Kurils with Hokkaido and also Sakhalin with the Asian mainland. They appeared several times during the Late Pleistocene and totally disappeared last time ca. 10000 BP.

Vegetation of the southern part of Russian Far East exhibits great variation from the boreal (taiga) forests do- 


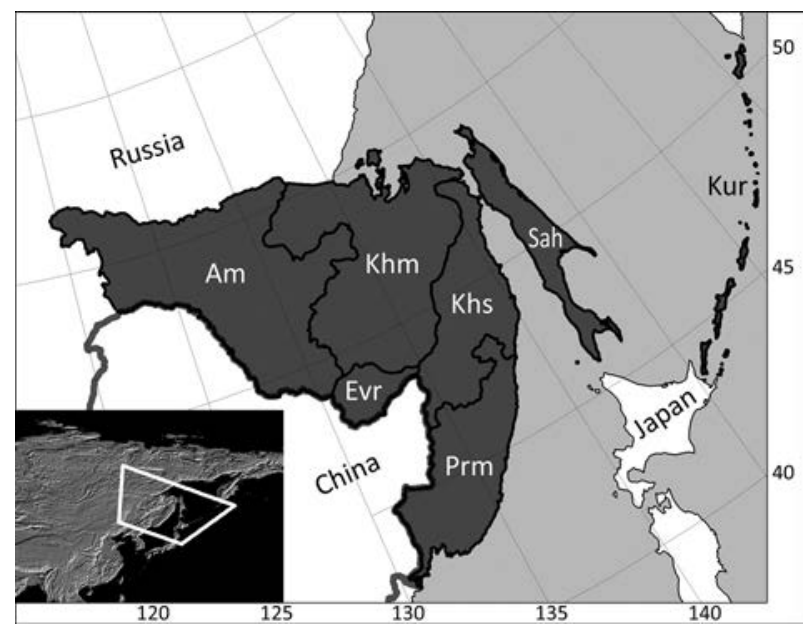

Figure 1 Regions of the Russian Far East: Evr - Evreiskaya [Jewish] Autonomous Province; Khm - Khabarovsk Territory, middle part, Khs - Khabarovsk Territory, southern part; Kur - Kuril Islands [Sakhalin Province]; Prm - Primorye Territory; Sah Sakhalin Province (excluding Kuril Islands)

minated by Picea, Pinus and Larix in the north of the studied area to the hemiboreal and cool temperate vegetation in the south. The upper elevations in the north are covered by mountain tundra, while southern mountains have only forestless communities without typical tundra elements. Oroboreal belt is receiving additional precipitation from the wet air masses going from the Pacific. The amazing feature of southern Far Eastern flora is a mixture of southern and northern flora elements: Abies trees are often embraced with Actinidia kolomicta and grow among Araliaceae, Phellodendron, evergreen shrubs of Skimmia, Ilex, Sasa, etc.

Bryological studies in the south of the Russian Far East started in the middle 19th century, with exploration of the territory by K.I. Maximovich, F.B. Schmidt and L.I. Schrenk. Some data on bryophytes were firstly published by Regel (1861) and Schmidt (1869); however, the work of S.O. Lindberg (1872), based on collections made by F.B. Schmidt and P.P. Glehn, may be regarded as the first serious input to the understanding of bryophyte flora of the region. These and some other data of that time were summarized by C. Warnstorf $(1913,1914)$ in his "Zur BryoGeographie der Russischen Reiches", which obviously showed the incompleteness of available information on moss diversity in the South of the Russian Far East. The important contribution to the Amur flora was the checklist prepared by Brotherus et al. (1916), basing on collections of O.I. Kuzeneva and N.I. Prokhorov.

First professional bryologist who collected bryophytes in the area was J. Podperra, who however worked here only a short time in February and March of 1920, and his list contains 72 species only (Podpera 1929). First really comprehensive study of the moss flora of Russian Far East was conducted by A.S. Lazarenko, who collected bryophytes in Primorsky Territory in 1930, 1933 and 1935. He has published the "Brief manual of leafy mosses of the Far East” (Lazarenko, 1938) with the first identification key for mosses of the Soviet Far East. Lazarenko also compiled the first (and last until the present account) detailed checklist of the Russian (Soviet in that time) Far East (Lazarenko, 1940, 1941a, 1941b, 1946), where all literature references available at that time were provided. Bryophyte flora of Sakhalin and Kuril Islands was also studied by Japanese scientists, S. Sugawara (1956) and Y. Horikawa (1940); bryophyte collections were also made on Sakhalin by Reverend U. Faurie (see Bakalin \& Katagiri 2014 for details).

$$
* * *
$$

The first professional bryologist who lived in Vladivostok and devoted all her life to study of moss diversity and taxonomy in the Russian Far East was Dr. Valentina Yakovlevna Cherdantseva (1939-2013), who prepared the first draft of the present checklist. It includes both published and unpublished materials. Most prominent studies of Cherdantseva concern bryophyte flora of Sakhalin and Primorsky Territory (the latter study was done together with the Dr. L.V. Bardunov). She also organized first moss herbarium in the Russian Far East. Her life and achievements are briefly described in her obituary (Bakalin 2013). After sudden death of Cherdantseva in 2013, Bakalin, her colleague in the Institute of Biology and Soil Sciences, started to revise her unpublished materials. Among others, there was a database in Excel file containing data, both published and unpublished earlier, on distribution of mosses in southern part of the Russian Far East. The authors of the present paper attempted to complete this work and publish it, supplemented by novelties discovered already after Cherdantseva passed away. Recent nomenclatural and taxonomic changes are mostly accepted, and synonyms used by Cherdantseva are given.

\section{CHECK - L IST}

The check-list includes 816 species and 10 infraspecific taxa with references on their distribution within seven floristic regions according to subdivisions accepted in the Moss flora of Russia (Ignatov et al. 2017). The nomenclature follows mostly Russian checkist [90], with additions accepted in Moss flora of Russia [97, 99].

We provide mainly the selected publications for respective regions [editor: for this reason the editorial board desided to numerate the list of references in this paper], mostly those that summarize previously published records, thus the check-list does not cite all papers where a taxon is mentioned in a given region. Recent taxonomic treatments of some groups of mosses were also taken into account, providing amended data on species names and distribution. In some cases, mostly for the species rare in the region, unpublished data of the authors of the present paper are also cited, referring to herbaria were they are deposited (more detailed information for these references is available in the database of the project "Moss flora of Russia" (http:// arctoa.ru/Flora/basa.php).

Species names are followed by synonymy where ever necessary, given in parenthesis, then the region abbreviations (cf. Fig. 1) are given with respective references. Species commented for their taxonomy and distribution are marked 
with (!!). Comments are given on taxonomic status of the species and on doubtful records in some regions. Eight species which are doubtful for all region are given with question mark (?). After the list of taxa that are confirmed or doubtful, but nevertheless may be present in the area (commonly no vouchers found, but occurrence of the taxon is somewhat possible in the Russian Far East), we place the list of excluded taxa with corresponding explanations.

Abietinella Müll. Hal. [Thuidiaceae]

- abietina (Hedw.) M. Fleisch.: Am [4, 7, 8, 32, 56, 70, 133], Khm [41, 43, 88], Khs [40, 65, 109, 123, 131, 163], Evr [39], Prm [26, 28, 29, 38, 42, 48, 133], Sah [24], Kur $[23,27]$.

Actinothuidium (Besch.) Broth. [Thuidiaceae]

- bookeri (Mitt.) Broth. subsp. boreale Ignatov, Ignatova, Z. Iwats. \& B.C. Tan: Khm [88].

Aloina Kindb. [Pottiaceae]

- rigida (Hedw.) Limpr.: Prm [MHA, 59].

Amblystegium Bruch, Schimp. \& W. Gümbel [Amblystegiaceae]

- radicale (P. Beauv.) Bruch, Schimp. \& W. Gümbel (A. saxatile Schimp., Campylium radicale (P. Beauv.) Grout): Prm [26] - (!!).

- serpens (Hedw.) Bruch, Schimp. \& W. Gümbel: Am [7, 8, 32, 56, 70], Khm [41, 88], Khs [40, 109, 163], Evr [39], Prm [26, 28, 29, 38, 42, 71], Sah [24, 36], Kur [21, 23, 25].

- serpens var. juratzkanum (Schimp.) Rau \& Herv. (A. juratrkanum Schimp.): Am [7, 8], Prm [26], Kur [21] - (!!).

Amphidium Schimp. [Rhabdoweisiaceae]

- asiaticum Sim-Sim, Afonina \& M. Stech: Am [56], Khm [145].

- lapponicum (Hedw.) Schimp.: Am [7, 8, 32, 56], Khm [88], Khs [65, 109], Prm [26, 28], Sah [24], Kur [21, 23, 25].

- mougeotii (Bruch \& Schimp.) Schimp.: Am [32, 56], Khm [88], Khs [163], Kur [23].

Anacamptodon Brid. [Amblystegiaceae]

- kamtchaticus Czernyadjeva: Sah [24, 53], Kur [23].

- latidens (Besch.) Broth.: Am [53], Khm [53, 88], Khs [109], Prm [26, 28, 29, 38, 42, 53, 133], Sah [24, 36, 53], Kur [23].

Andreaea Hedw. [Andreaeaceae]

- alpestris (Thed.) Bruch, Schimp. \& W. Gümbel: Khs [65], Sah [36], Kur [27, 25, 141].

- blyttii Bruch et al.: Kur [23].

- nivalis Hook.: Kur [21, 25, 141].

- obovata Thed.: Kur [23].

- rupestris Hedw: Am [7, 8, 56, 130], Khm [43, 88], Khs [40, 65, 109], Prm [26, 38, 48], Sah [24], Kur [21, 23, 25, 141].

Anoectangium Schwägr. [Pottiaceae]

- thomsonii Mitt.: Am [32, 56, 100], Khm [43, 100], Khs $[65,109]$, Prm [26, 28, 29, 71, 100], Kur [23] - (!!).

Anomobryum Schimp. [Bryaceae]

- bavaricum (Warnst.) Holyoak \& Köckinger: Prm [55].

- concinnatum (Spruce) Lindb. (A. filiforme auct.): Am [32, 55, 56], Khm [55], Prm [26, 55], Sah [24, 55], Kur [21, $23,25,55]$.

- nitidum (Mitt.) A. Jaeger: Am [56], Prm [55], Kur [25].

Anomodon Hook \& Taylor [Anomodontaceae]

- giraldii Müll. Hal.: Khs [40], Evr [39], Prm [26, 28, 29, 48, 71, 132], Sah [24, 36], Kur [23, 27].
- longifolius (Brid.) Hartm.: Am [132], Khm [41], Prm $[26,28]$, Sah [24, 36], Kur [23, 27].

- minor (Hedw.) Fürnr.: Am [4, 7, 8, 32, 56, 70], Khm [41, 88], Khs [40, 109, 123, 131, 163], Prm [26, 28, 29, 38, 42, 48, 71], Kur [23].

- rugelii (Müll. Hal.) Keissl.: Am [32], Khm [41], Khs [40, 109, 131, 163], Evr [39], Prm [26, 28, 29, 38, 42, 48, 71, 132], Sah [24, 36], Kur [23, 27].

- solovjovii Laz.: Prm [26, 28, 42, 71, 132, MHA].

- thraustus Müll. Hal.: Am [4, 56, 70, 132], Khm [41, 88, 131], Khs [40, 109, 123, 131], Evr [4, 39], Prm [26, 28, 29, 38, 42, 71, 132], Sah [24, 36], Kur [23, 27].

Archidium Brid. [Archidiaceae]

- alternifolium (Hedw.) Mitt.: Prm [3, 26, MHA].

Arctoa Bruch, Schimp. \& W. Gümbel [Rhabdoweisiaceae] - fulvella (Dicks.) Bruch, Schimp. \& W. Gümbel: Khm [88], Khs [65], Prm [144], Kur [21, 23, 141].

Arrbenopterum Hedw. [Aulacomniaceae]

- heterostichum Hedw. (Aulacomnium heterostichum (Hedw.) Bruch \& Schimp.): Am [4, 70], Khm [41], Khs [40, 109, 123, 131], Evr [39], Prm [26, 28, 29, 38, 42, 48], Sah [24, 36], Kur [23, 25].

Atrichum P. Beauv. [Polytrichaceae]

- flavisetum Mitt. (A. haussknechtii Jur. \& Milde): Am [8, 32, 56, 70, 130], Khm [41, 88], Khs [40, 109, 131], Evr [39], Prm [26, 28, 29, 38, 42, 48, 130], Sah [24, 36], Kur [23, 25].

- rhystophyllum (Müll. Hal.) Paris: Evr [39], Prm [26, 97].

- tenellum (Röhl.) Bruch, Schimp. \& W. Gümbel: Am [56], Kur [27] - (!!).

- undulatum (Hedw.) P. Beauv.: Am [35, 56], Khm [MHA], Khs $[40,123,131]$, Prm [26, 28, 48, 71], Sah [24, 36], Kur [23, 25].

Aulacomnium Schwägr. [Aulacomniaceae]

- beterostichum (Hedw.) Bruch \& Schimp., see Arrhenopterum beterostichum.

- palustre (Hedw.) Schwägr.: Am [4, 7, 8, 32, 35, 56, 70], Khm [41, 43, 88], Khs [40, 65, 109, 123, 163], Evr [39], Prm [26, 28, 29, 38, 42, 71], Sah [24, 36], Kur [21, 23, 25, 27, 141].

- turgidum (Wahlenb.) Schwägr.: Am [4, 7, 8, 32, 35, 56], Khm [41, 43, 88], Khs [65, 109, 123], Prm [38, 130], Sah [24, 36], Kur [21].

Barbula Hedw. [Pottiaceae], see also Hydrogonium and Streblotichum

- unguiculata Hedw.: Am [32], Khm [88], Prm [26, 130], Sah [24], Kur [23].

Bartramia Hedw. [Bartramiaceae]

- deciduaefolia Broth. \& Yasuda: Khm [99, MHA], Prm [99].

- ithyphylla Brid.: Am [32, 56], Khm [88], Khs [65], Prm [99, MHA], Sah [24], Kur [21].

- pomiformis Hedw.: Am [4, 7, 8, 32, 35, 56, 70, 131], Khm $[41,88]$, Khs $[40,65,109,123,131]$, Evr [39], Prm [26, 28, 29, 38, 42, 48, 71, 131], Sah [24, 36], Kur [23, 25, 27].

Bartramiopsis Kindb. [Polytrichaceae]

- lescurii (James) Kindb.: Khm [88], Khs [65, 123], Prm $[48,130]$, Sah [24, 36], Kur [23, 25, 27, 141].

Blindia Bruch, Schimp. \& W. Gümbel [Seligeriaceae]

- acuta (Hedw.) Bruch, Schimp. \& W. Gümbel: Am [7, 8, 32, 56], Khm [88], Khs [65], Prm [MHA], Sah [24], Kur [23].

Blindiadelphus (Lindb.) Fedosov \& Ignatov [Seligeriaceae] - diversifolius (Lindb.) Fedosov \& Ignatov (Seligeria diversifolia Lindb.): Am [97], Khs [65], Prm [97], Sah [97].

- recurvatus (Hedw.) Fedosov \& Ignatov (Seligeria recurvata 
(Hedw.) Bruch, Schimp. \& W. Gümbel): Prm [26] - (!!). - sibiricus (Lindb.) Fedosov \& Ignatov: Khs [67].

Boulaya Cardot [Thuidiaceae]

- mittenii (Broth.) Cardot: Khm [41], Khs [40], Prm [26, $28,29,38,48,71,132]$, Sah [24, 36], Kur [23, 25, 27].

Brachydontium Fürnr. [Seligeriaceae]

- olympicum (E. Britton) McIntosh \& Spense: Kur [21].

Brachymenium Schwägr. [Bryaceae]

- exile (Dozy \& Molk.) Bosch \& Sande Lac. (B. exiloides Bard. \& Czerd.): Prm [26, 99].

- nepalense Hook.: Am [70], Evr [39], Prm [26, 71, 132, $\mathrm{MHA}]$.

Brachytheciastrum Ignatov \& Huttunen [Brachytheciaceae] - trachypodium (Brid.) Ignatov \& Huttunen: Am [56, 132], Khs $[65,109]$.

- velutinum (Hedw.) Ignatov \& Huttunen: Khs [123], Prm [26, 29, 38, 132], Sah [24, 36], Kur [23].

Brachythecium Bruch, Schimp. \& W. Gümbel [Brachytheciaceae]

- albicans (Hedw.) Bruch, Schimp. \& W. Gümbel: Kur [21, 86] - (!!)

- auriculatum A. Jaeger (Camptothecium auriculatum (A. Jaeger) Broth.): Khs [163], Prm [26, 28, 48, 86, 132], Sah $[24,36,86]$, Kur [23, 25, 27].

- baicalense Ignatov: Am [32, 93], Khm [93], Khs [109], Prm [86, 93], Sah [24], Kur [113].

- buchananii (Hook.) A. Jaeger: Am [32, 56, 70], Khm [88], Khs [109], Evr [39], Prm [29, 38, 48], Sah [24], Kur [23].

- campestre (Müll. Hal.) Bruch, Schimp. \& W. Gümbel: Sah [24, 36], Kur [23, 86] - (!!).

- cirrosum (Schwägr.) Schimp.: Am [56, 132], Khm [88], Khs [65], Sah [24].

- complanatum Broth.: Am [32, 70, 93], Khm [93, 88], Khs [93, 109], Evr [39], Prm [38, 86, 93].

- coruscum I. Hagen: Sah [24, 86].

- daburicum Ignatov: Am [32, 56 ], Khs [109].

- erythrorrhizon Bruch, Schimp. \& W. Gümbel (Brachythecium thedenii Bruch, Schimp. \& W. Gümbel): Am [56, 86], Khm [86, 88], Prm [26, 71, 86], Sah [24, 86]

- extremiorientale Ignatov: Prm [93], Sah [24, 86], Kur $[23,86]$.

- garovaglioides Müll. Hal.: Khs [65].

- belminthocladum Broth. \& Paris: Prm [86].

- bultenii (E.B. Bartram) Min Lin \& Y.F. Wang (Brybnia hultenii E.B. Bartram, Brachythecium novae-angliae subsp. bultenit (E.B. Bartram) Huttunen): Am [8, 32, 70], Khm [41, 88], Khs [40], Evr [39], Prm [26, 28, 29, 38, 42], Sah [24, 36, 79], Kur [21, 27, 141].

- irinae Ignatov: Khs [109], Sah [24], Kur [86].

- jacuticum Ignatov: Khs [109].

- kuroishicum Besch.: Prm [26, 28, 29, 86], Sah [24, 36], Kur $[23,25,26,86]$.

- mildeanum (Schimp.) Schimp.: Am [7, 8, 32, 56], Khm [88, 132], Khs [109], Prm [26, 28, 42, 71, 132], Sah [24], Kur [23, 25].

- noesicum Besch. (Brybnia noesica (Besch.) Broth.): Prm [79], Sah [24, 79], Kur [23, 25, 79, 113].

- rivulare Bruch, Schimp. \& W. Gümbel: Am [7, 8, 32, 35, 56, 70, 132], Khm [88], Khs [40, 109], Evr [39], Prm [26, 28, 29, 38, 42, 48, 71], Sah [24], Kur [21, 23, 25, 27 .

- rotaeanum De Not.: Am [32, 56, 86], Khm [88], Khs [109], Evr [39], Prm [29, 38, 48, 71], Sah [24], Kur [23]. - rutabulum (Hedw.) Bruch, Schimp. \& W. Gümbel: Khs [123, 131], Prm [28], Kur [23, 25] - (!!).
- salebrosum (F. Weber \& D. Mohr) Bruch, Schimp. \& W. Gümbel: Am [7, 8, 35, 56, 70], Khm [41, 88], Khs [40, 109, 123], Prm [26, 28, 29, 42], Sah [24, 36], Kur [27] - (!!). - udum I. Hagen: Kur [23].

Breidleria Loeske [Pylaisiaceae]

- pratensis (W.D.J. Koch ex Spruce) Loeske (Hypnum pratense W.D.J. Koch ex Spruce): Khs [123], Prm [26, 38], Sah $[24,36]$, Kur [128].

Brothera Müll. Hal. [Leucbryaceae]

- leana (Sull.) Müll. Hal.: Am [4, 7, 8, 32, 56, 70, 130], Khm [41, 88], Khs [40, 109, 123], Evr [4, 39], Prm [26, $28,29,38,42,48,71,130]$.

Brotherella Loeske ex M. Fleisch. [Pylaisiadelphaceae] - henonii (Duby) M. Fleisch.: Kur [23, 27].

Bryhnia Kaurin [Brachytheciaceae], see Brachythecium hultenii, B. noesicum, Myuroclada longiramea

Bryochenea C. Gao \& G.C. Zhang [Thuidiaceae]

- vestitissima (Besch.) Touw (Thuidium vestitissimum Besch.): Prm [5, 26, 28, 132], Kur [23].

Bryoerythrophyllum P.C. Chen [Pottiaceae]

- alpigenum (Venturi) P.C. Chen: Kur [23, 61].

- brachystegium (Besch.) K. Saito: Am [32], Sah [24], Kur $[23,25,61]$.

- ferruginascens (Stirt.) Giacom.: Am [32, 56], Khm [61, 88], Prm [MHA], Sah [24].

- inaequalifolium (Taylor) R.H. Zander: Am [32], Prm [59, MHA].

- recurvirostrum (Hedw.) P.C. Chen: Am [8, 32, 34, 56, 61, 70], Khm [61, 88], Khs [40, 109, 131], Evr [39], Prm [26, 28, 29, 38, 42, 61], Sah [24], Kur [21, 23, 25, 61].

- rubrum (Jur. ex Geh.) P.C. Chen: Sah [24].

Bryonoguchia Z. Iwats. \& Inoue [Thuidiaceae]

- molkenboeri (Sande Lac.) Z. Iwats. \& Inoue: Am [70], Evr [39], Prm [26, 28, 42, 48, 71], Sah [77].

Bryoxiphium Mitt. [Bryoxiphiaceae]

- japonicum (Berggr.) E. Britton (B. savatieri (Husn.) Mitt.): Khs [64, 109], Evr [39, 64], Prm [26, 29, 64], Sah [24, 64], Kur [21, 23, 25, 27, 64, 141].

- norvegicum (Brid.) Mitt.: Kur [64].

Bryum Hedw. [Bryaceae]

- algovicum Sendtn. ex Müll. Hal. (B. angustirete Kindb.): Am [32, 164], Khs [109, 131], Prm [99, MHA], Sah [24].

- altaicum Broth.: Kur [99, MHA, VLA].

- amblyodon Müll. Hal. (B. inclinatum (Hedw.) Dicks.): Am [32, 164], Khm [99, MHA], Khs [109], Prm [26, 38, 164], Sah [24, 36, 164], Kur [21, 23, 164].

- archangelicum Bruch, Schimp. \& W. Gümbel: Prm [164], Kur [23].

- argenteum Hedw: Am [7, 8, 32, 56], Khm [88], Khs [109, 123], Prm [26, 28, 29, 42, 71, 164], Sah [24, 36], Kur [21, 23, 164].

- bimum (Schreb.) Turner: Prm [164], Sah [24], Kur [23, 164].

- caespiticium Hedw: Am [8, 32], Khm [88], Khs [109, 123], Prm [28], Sah [24, 36, 164].

- capillare Hedw.: Am [32, 35], Khs [109], Prm [26, 28, 29 , 164], Sah [24, 36, 164], Kur [23, 164].

- creberrimum Taylor: Am [7, 8, 56], Khs [109], Prm [164], Sah [24, 164], Kur [21, 23, 164].

- cryophilum Mårtensson: Khm [88].

- cyclophyllum (Schwägr.) Bruch \& Schimp.: Am [32, 56, 164], Khm [88], Prm [28, 164], Kur [164].

- dichotomum Hedw. (Bryum bicolor Dicks.): Prm [26]. 
- elegans Nees: Am [56], Khm [88], Khs [109], Prm [26, 164], Sah [24, 36], Kur [164].

- funckii Schwägr.: Prm [26, 164].

- intermedium (Brid.) Blandow: Kur [23].

- lonchocaulon Müll. Hal.: Am [8, 32].

- mayrii Broth.: Kur [21].

- mildeanum Jur.: Am [32, 164].

- moravicum Podp.: Khm [88], Khs [109], Prm [29, 164], Sah [24], Kur [23].

- neodamense Itzigs.: Prm [164].

- pallens Sw. ex anon.: Am [8, 32], Prm [26, 28, 29, 42, 164], Sah [24, 36, 164].

- pseudotriquetrum (Hedw.) G. Gaertn. B. Mey. \& Scherb. (B. pseudocrispulum (Podp.) L. Savicz): Am [7, 8, 32, 56], Khm [88], Khs [65, 109], Evr [39], Prm [26, 28, 29, 38, 42, 164], Sah [24, 164], Kur [23, 27, 141, 164].

- radiculosum Brid.: Prm [29], Kur [23, 164].

- salinum I. Hagen ex Limpr.: Sah [24, 36, 164], Kur [23].

- schleicheri DC.: Prm [164], Sah [24, 164], Kur [21, 23].

- subapiculatum Hampe (B. erythrocarpum Schwägr.): Prm [26, 164].

- uliginosum (Brid.) Bruch \& Schimp.: Prm [29], Sah [24, 36, 164].

- violaceum Crundw. \& Nyholm: Prm [164].

- weigelii Spreng.: Am [34], Sah [24, 164], Kur [23].

Bucklandiella Roiv. [Grimmiaceae]

- laeta (Besch. \& Cardot) Bedn.-Ochyra \& Ochyra (Racomitrium laetum Besch. \& Cardot): Khm [88], Prm [38], Kur [23, 25].

- microcarpa (Hedw.) Bedn.-Ochyra \& Ochyra (Racomitrium microcarpum (Hedw.) Brid.): Am [56, 97], Khm [43, 88], Khs [65], Evr [97], Prm [26], Sah [24], Kur [23, 25].

- nitidula (Cardot) Bedn.-Ochyra \& Ochyra (Racomitrium nitidulum Cardot): Am [56], Kur [23].

- sudetica (Funck) Bedn.-Ochyra \& Ochyra (Racomitrium sudeticum (Funck) Bruch, Schimp. \& W. Gümbel): Am [8, 34, 56], Khm [88], Khs [40], Evr [97], Sah [24], Kur [21, $23,25,141]-(! !)$.

Buxbaumia Hedw. [Buxbaumiaceae]

- aphylla Hedw.: Am [8, 43], Khm [88], Khs [131], Sah $[24,36]$, Kur [141].

- minakatae S. Okamura: Am [4], Khs [65], Prm [26, 28, $29,42,71]$.

Callicladium H.A. Crum [Pylaisiaceae]

- haldanianum (Grev.) H.A. Crum (Heterophyllium haldanianum (Grev.) M. Fleisch.): Am [8, 32, 35, 56, 70], Khm [41, 88], Khs [40, 109, 123, 131, 163], Evr [39], Prm [26, 28, 29, 38, 42, 48, 71], Sah [24, 36], Kur [23, 25, 27].

Calliergon (Sull.) Kindb. [Calliergonaceae]

- cordifolium (Hedw.) Kindb.: Am [4, 7, 8, 32, 56, 70], Khm [41, 43, 88], Khs [40, 109], Evr [39], Prm [26, 28, 29, 38, 42], Sah [24, 36], Kur [21, 23, 25, 141].

- giganteum (Schimp.) Kindb.: Am [4, 7, 8, 32, 56], Khm [88], Khs [123], Prm [26, 28], Sah [24, 36], Kur [25].

- megalophyllum Mikut.: Am [32].

- richardsonii (Mitt.) Kindb.: Am [7, 8, 35, 32, 43, 56], Khm [41, 88], Sah [24], Kur [21].

Calliergonella Loeske [Pylaisiaceae]

- cuspidata (Hedw.) Loeske: Am [32, 56, 70], Khs [40], Prm [26, 28, 29, 38, 71], Sah [24, 36], Kur [23, 25, 27].

- lindbergii (Mitt.) Hedenäs (Hypnum lindbergii Mitt.): Am [4, 7, 8, 32, 35, 56, 70], Khm [41, 43, 88], Khs [40, 109, 123, 131], Evr [39], Prm [26, 28, 29, 38, 42, 48, 71], Sah $[24,36]$, Kur [21, 23, 25, 141$]$.
Campyliadelphus (Kindb.) R.S. Chopra [Amblystegiaceae]

- chrysophyllus (Brid.) Kanda (Campylium chrysophyllum (Brid.) Lange): Am [4, 8, 32, 56], Khs [40, 123, 163], Evr [39], Prm [26, 29, 28], Sah [24], Kur [23, 25].

- elodes (Lindb.) Kanda (Campylium elodes (Lindb.) Kindb.): Prm [26, 132], Kur [23].

Campylophyllopsis W.R. Buck (Campylidium (Kindb.) Ochyra) [Amblystegiaceae]

- bispidulum (Brid.) Ochyra (Campylium hispidulum (Brid.) Mitt., Campylidium hispidulum (Brid.) Ochyra): Am [8], Khm [41, 88], Khs [40], Prm [26, 28, 29, 42, 71] - (!!)(?).

- sommerfeltii (Myrin) Ochyra (Campylium sommerfeltii (Myrin) Lange, Campylidium sommerfeltii (Myrin) Ochyra): Am [32, 34, 56, 70], Khm [41, 88], Khs [40, 109, 123, 131], Evr [39], Prm [26, 28, 38, 48], Sah [24, 36], Kur [23].

Campylium (Sull.) Mitt. [Amblystegiaceae]

- protensum (Brid.) Kindb.: Am [32], Prm [29], Kur [21, 23, 25].

- squarrosulum (Besch. \& Cardot) Kanda: Prm [28, MHA], Kur [23].

- stellatum (Hedw.) C.E.O. Jensen: Am [32, 56, 70], Khm [88], Khs [65], Prm [26, 29, 38], Sah [24], Kur [25].

Campylophyllum (Schimp.) M. Fleisch. [Amblystegiaceae] - halleri (Hedw.) M. Fleisch.: Sah [24].

Campylopus Brid. [Leucobryaceae]

- fragilis (Brid.) Bruch, Schimp. \& W. Gümbel: Am [32] - (!!).

- introflexus (Hedw.) Brid.: Prm [59].

- pyriformis (Schultz) Brid.: Khs [130], Prm [26, 48, 130], Kur [25].

- schimperi Milde: Khm [88].

- subulatus Schimp. ex Milde: Prm [59, MHA].

Catoscopium Brid. [Catoscopiaceae]

- nigritum (Hedw.) Brid.: Sah [24].

Ceratodon Brid. [Ditrichaceae]

- purpureus (Hedw.) Brid.: Am [7, 8, 32, 35, 56, 70], Khm [41, 43, 88], Khs [40, 65, 109, 123, 163], Evr [4, 39], Prm [26, 28, 29, 38, 42, 48, 71], Sah [24, 36], Kur [21, 23, 25, 141].

Cinclidium Sw. [Mniaceae]

- latifolium Lindb.: Kur [141].

Cirriphyllum Grout [Brachytheciaceae]

- piliferum (Hedw.) Grout: Am [8], Khs [109], Prm [26], Kur [21, 23, 25].

Claopodium (Lesq. \& James) Renauld \& Cardot [Leskeaceae]

- pellucinerve (Mitt.) Best: Am [4, 32, 56, 70], Khm [41, 88], Khs [40, 109, 123], Evr [4, 39], Prm [26, 28, 29, 38, 42, 71], Sah [24, 36], Kur [23, 25].

Climacium F. Weber \& D. Mohr [Climaciaceae]

- dendroides (Hedw.) F. Weber \& D. Mohr: Am [4, 7, 8, 32, 35, 56, 70], Khm [43, 88, 131], Khs [109, 163], 39, Prm [26, 28, 29, 38, 48, 71], Sah [24, 36], Kur [21, 23, 25].

- japonicum Lindb.: Am [70], Khm [41, 131], Khs [40, 65, 109, 123, 131], Evr [39], Prm [26, 28, 29, 38, 42, 48, 71], Sah [36], Kur [23, 27].

Cnestrum I. Hagen [Rhabdoweisiaceae]

- alpestre (Wahlenb.) Nyholm: Khm [88], Khs [65, 131], Sah [24].

- glaucescens (Lindb. \& Arnell) Holmen ex Mogensen \& Steere (Cynodontium glaucescens (Lindb. \& Arnell) Paris): Am [8], Prm [26].

- schistii (F. Weber \& D. Mohr) I. Hagen: Am [8, 32, 56], 
Khm [88], Khs [109, 131], Prm [26], Sah [24].

Codriophorus P. Beauv. [Grimmiaceae], see also Dilutineuron

- acicularis (Hedw.) P. Beauv.: Kur [23, 141] - (!!).

- carinatus (Cardot) Bedn.-Ochyra \& Ochyra: Prm [45], Kur [23].

- mollis (Cardot) Bedn.-Ochyra \& Ochyra: Kur [23, 25].

Conardia H. Rob. [Calliergonaceae]

- compacta (Drumm. ex Müll. Hal.) H.Rob.: Kur [23, 27].

Conostomum Sw. ex F. Weber \& D. Mohr [Bartramiaceae]

- tetragonum (Hedw.) Lindb. - Khm [88], Khs [65].

Coscinodon Spreng. [Grimmiaceae]

- cribrosus (Hedw.) Spruce: Am [56], Khm [MW], Prm [29], Sah [24] - (!!)

- pseudohartzii Hastings, Ignatova \& Köckinger: Prm [105].

- yukonensis Hastings: Khm [105], Prm [105], Sah [24], Kur [23, 105]

Cratoneuron (Sull.) Spruce [Amblystegiaceae]

- filicinum (Hedw.) Spruce: Am [7, 8, 32, 56], Khs [109, 163], Evr [39], Prm [26, 28, 29, 38, 42, 71], Sah [24, 36], Kur $[21,23,25,27,141]$

Cryphaea D. Mohr. [Cryphaeaceae]

- amurensis Ignatov: Am [8, 56], Khm [81, 88], Khs [109], Prm [45].

Cynodontium Bruch \& Schimp. [Rhabdoweisiaceae]

- asperifolium (Lindb. \& Arnell) Paris: Am [8, 32, 56, 130], Khs [109, 123], Prm [26, 28, 130], Sah [24].

- polycarpon (Hedw.) Schimp.: Am [8], Prm [26, 29] - (!!)(?).

- strumiferum (Hedw.) Lindb.: Am [7, 8, 56], Khm [88], Khs [65, 109, 123], Prm [26, 28], Sah [24], Kur [25].

- tenellum (Schimp.) Limpr.: Am [8, 56, 130], Khm [43, 88], Khs [109, 131], Prm [26], Sah [24], Kur [113].

Cyrto-hypnum (Hampe) Hampe \& Lorentz. [Thuidiaceae] see Pelekium

Cyrtomnium Holmen [Mniaceae]

- hymenophylloides (Huebener) T.J. Kop.: Am [57], Khm [88], Khs [65], Prm [MHA], Sah [24].

- hymenophyllum (Bruch, Schimp. \& W. Gümbel) Holmen: Sah [99], Kur [21].

Dichelyma Myrin [Fontinalaceae]

- japonicum Cardot: Kur [23, 54].

Dichodontium Schimp. [Rhabdoweisiaceae], see also Diobelonella

- pellucidum (Hedw.) Schimp.: Am [8, 32, 56], Khm [88], Khs [109], Prm [26, 29, 38], Sah [24, 36], Kur [21, 23, $25,113,141]$.

Dicranella (Müll. Hal.) Schimp. [Dicranaceae], see also Diobelonella

- cerviculata (Hedw.) Schimp.: Am [7, 8, 56], Khm [88], Khs [40], Prm [72], Sah [24, 36], Kur [23, 25, 27].

- crispa (Hedw.) Schimp.: Am [8, 32, 130], Khm [88], Kur [25].

- curvipes (Lindb.) Ignatov: Am [56], Khs [65], Sah [24, 36], Kur [23, 25].

- grevilleana (Brid.) Schimp.: Sah [24].

- heteromalla (Hedw.) Schimp.: Am [32, 70], Khm [41, 88], Khs [40, 109, 131, 163], Evr [39], Prm [26, 28, 29, 38, 42, 48, 71, 130], Kur [27].

- humilis R. Ruthe (Anisothecium rigidulum (Sw. ex Hedw.) C.E.O. Jensen): Am [32], Prm [26, 28, 130], Sah [24].
- rufescens (Dicks.) Schimp. (Anisothecium rufescens (Dicks.) Lindb.): Khs [131], Prm [26, 130], Kur [27, 25].

- schreberiana (Hedw.) Hilf. ex H.A. Crum \& L.E. Anderson (Anisothecium schreberianum (Hedw.) Dixon): Am [7, 8, 32], Khm [88], Khs [65], Prm [26], Sah [24], Kur [23].

- subsecunda Besch.: Sah [90] - (!!)(?).

- subulata (Hedw.) Schimp.: Am [7, 8, 32, 130], Khm [88], Khs [65, 109], Prm [72, MHA], Sah [24, 36], Kur [21, 23, $25,27,141]$.

- varia (Hedw.) Schimp. (Anisothecium varium (Hedw.) Mitt.): Am [32], Khm [88], Prm [26, 71], Sah [24], Kur [25, 113].

Dicranodontium Bruch, Schimp. \& W. Gümbel [Leucobryaceae]

- asperulum (Mitt.) Broth.: Khm [88].

- denudatum (Brid.) E. Britton: Khm [41, 88], Khs [40, 65, 109, 123], Evr [39], Prm [26, 38, 42, 130], Sah [36], $\operatorname{Kur}[23,25,27,113]$.

Dicranoloma (Renauld) Renauld [Dicranaceae]

- cylindrothecium (Mitt.) Sakurai: Prm [45], Kur [IRK, MW].

Dicranum Hedw. [Dicranaceae]

- acutifolium (Lindb. \& Arnell) C.E.O. Jensen: Am [7, 8, 56], Khm [88], Khs [163], Sah [24], Kur [25].

- angustum Lindb.: Am [8, 130], Khm [88], Khs [65, 123].

- bardunovii Tubanova \& Ignatova: Am [56, 154].

- bonjeanii De Not.: Am [4, 8, 56], Khm [41, 43, 88], Khs [65], Prm [29, 38], Sah [36], Kur [23, 25, 141].

- brevifolium (Lindb.) Lindb.: Am [32], Khm [88], Khs [163], Sah [24].

- dispersum Engelmark: Am [32], Prm [143], Kur [113].

- drummondii Müll. Hal.: Am [56], Khs [2, 109], Prm [2], Sah $[24,36]$.

- elongatum Schleich. ex Schwägr.: Am [7, 8, 56, 130], Khm [43, 88, 157], Khs [65, 109], Prm [130], Sah [24, 157], Kur [25].

- flagellare Hedw. (Orthodicranum flagellare (Hedw.) Loeske): Am $[4,7,8,32,56,70,130]$, Khm [41, 88], Khs [40, 65, 109, 123, 131], Evr [4, 39, 130], Prm [26, 28, 29, 38, 42, 71, 130], Sah [24, 36], Kur [23, 27].

- flexicaule Brid. (D. congestum Brid.): Am [7, 8, 35, 56, 130], Khm [41, 43], Khs [109, 131], Prm [26, 42], Sah [24], Kur [21].

- fragilifolium Lindb.: Am [4, 7, 8, 32, 56, 101], Khm [41, 43, 88, 101], Khs [40, 65, 109, 163], Evr [39].

- fulvum Hook.: Am [8], Prm [29, 130].

- fuscescens Turner: Am [7, 8, 32, 35, 56, 130], Khm [43, 88], Khs [65, 109, 123], Prm [26, 29, 38, 130], Sah [24, 36], Kur [21, 23, 25, 27].

- groenlandicum Brid.: Am [56, 130, 157], Khm [43, 157], Khs [157], Sah [24, 36, 157], Kur [23].

- hakkodense Cardot: Khm [101], Prm [29, 48, 101], Sah [24], Kur [23, 25].

- hamulosum Mitt.: Sah [24, 36], Kur [21, 23, 25].

- ignatovii Tubanova \& Fedosov: Khs [159], Sah [159], Kur [159, 152].

- japonicum Mitt.: Am [56], Khs [123], Sah [36], Kur [23].

- laevidens R.S. Williams: Sah [24], Kur [21].

- leioneuron Kindb.: Am [32], Khs [109], Prm [26], Sah [24], Kur [21].

- lorifolium Mitt.: Prm [128a] - (!!)

- majus Turner: Am [7, 8, 35, 56, 130], Khm [43, 88], Khs $[65,109,123,131,163]$, Prm $[26,29,38,71]$, Sah [24, 36], Kur [21, 23, 25, 27, 141].

- majus var. orthophyllum A. Braun ex Milde: Kur [21].

- mayrii Broth. (Orthodicranum mayrii (Broth.) Smirnova): Prm [26, 28, 48], Sah [24], Kur [23].

- montanum Hedw. (Orthodicranum montanum (Hedw.) 
Loeske): Am [7, 8, 32, 56], Khm [41, 88], Khs [65, 109, 123, 163], Evr [4, 39], Prm [26, 28, 29, 38, 42, 48, 71], Sah $[24,36]$.

- mueblenbeckii Bruch, Schimp. \& W. Gümbel: Am [7, 8], Khs [123]. 130].

- nipponense Besch.: Am [32, 56], Prm [28, 142], Sah [24], Kur [21, 23, 142].

- pacificum Ignatova \& Fedosov: Am [57], Khm [101], Sah [24, 101], Kur [23, 25, 101].

- polysetum Sw.: Am [7, 8, 32, 35, 56, 130], Khm [41, 43, 88], Khs [109, 123, 131], Prm [26, 38, 42, 48, 130], Sah $[24,36]$, Kur [25].

- schljakovii Ignatova \& Tubanova: Am [56], Khm [111], Khs [65]. Prm [111].

- scoparium Hedw.: Am [4, 7, 8, 35, 70, 32, 56, 130], Khm [41, 43, 88], Khs [40, 65, 109, 123, 131, 163], Evr [39], Prm $[26,28,29,38,42,48,71,130]$, Sah [24, 36], Kur $[21,23,27,141]$.

- septentrionale Tubanova \& Ignatova: Khs [65], Prm [155], Sah [24].

- setifolium Cardot: Khm [MW], Kur [23].

- spadiceum J.E. Zetterst.: Am [7, 8, 56], Khm [88], Prm [26, 130], Sah [24], Kur [21, 23, 25].

- undulatum Schrad. ex Brid.: Am [4, 7, 8, 32, 35, 56, 130], Khm [41, 43, 88], Khs [109, 123], Prm [38, 130], Sah [24, 36], Kur [21, 23, 25, 141].

Didymodon Hedw. [Pottiaceae]

- asperifolius (Mitt.) H.A. Crum, Steere \& L.E. Anderson: Am [32].

- brachyphyllus (Sull.) R.H. Zander: Kur [25].

- cordatus Jur. (Barbula cordata (Jur.) Loeske): Prm [26, 130].

- erosodenticulatus (Müll. Hal.) K. Saito: Prm [17].

- fallax (Hedw.) R.H. Zander: Sah [24].

- ferrugineus (Schimp. ex Besch.) M.O. Hill (Barbula reflexa (Brid.) Brid.): Am [32, 34, 56, 130], Khm [88], Prm [26, $28,130]$.

- glaucus Ryan: Khs [109], Prm [130], Sah [24].

- hedysariformis Otnyukova: Am [32, 56].

- icmadophilus (Schimp. ex Müll. Hal.) R.H. Zander: Am [56], Prm [MHA].

- insulanus (De Not.) M.O. Hill (Barbula cylindrica (Taylor) Schimp. ex Boulay): Prm [26, 130].

- maschalogena (Renauld \& Cardot) Broth.: Am [32], Prm [MHA].

- perobtusus Broth.: Sah [24].

- rigidulus Hedw. (Barbula rigidula (Hedw.) Milde): Am [8, 130], Khm [88], Prm [26, 28], Sah [24], Kur [23].

- spadiceus (Mitt.) Limpr. (Barbula spadicea (Mitt.) Braithw.): Prm [130, 147].

- tophaceus (Brid.) Lisa: Kur [23, 113].

- cf. validus Limpr. (Barbula. rigidula var. valida (Limpr.) Broth.): Am [32, 56], Prm [130, 26].

- vinealis (Brid.) R.H. Zander (Barbula vinealis Brid.): Prm [130]. - zanderi Afonina \& Ignatova: Am [56], Khs [109], Prm [12]. Dilutineuron Bedn.-Ochyra, Sawicki, Ochyra, Szczecińska \& Plášek [Grimmiaceae]

- brevisetum (Lindb.) Bedn.-Ochyra, Sawicki, Ochyra, Szczecińska \& Plášek (Racomitrium brevisetum Lindb., Codriophorus brevisetus (Lindb.) Bedn.-Ochyra \& Ochyra): Am [97], Khm [97], Khs [40, 65], Evr [39], Prm [48], Sah [24, 36], Kur [21, 23, 25, 141].

- corrugatum (Bedn.-Ochyra) edn.-Ochyra, Sawicki, Ochyra, Szczecińska \& Plášek (Codriophorus corrugatus Bedn.Ochyra): Sah [24], Kur [23, 25, 141].

- fasciculare (Hedw.) Bedn.-Ochyra, Sawicki, Ochyra, Szczecińska \& Plášek (Racomitrium fasciculare (Schrad. ex Hedw.) Brid.): Khm [43, 88], Khs [123], Prm [38, 48], $\operatorname{Kur}[21,23,25,141]$.
Diobelonella Ochyra [Dicranaceae]

- palustris (Dicks.) Ochyra (Anisothecium palustre (Dicks.) I. Hagen, Dichodontium palustre (Dicks.) M. Stech, Dicranella palustris (Dicks.) Crundw.): Kur [21, 23, 25, 27, 141].

Diphyscium D. Mohr [Diphysciaceae]

- foliosum (Hedw.) D. Mohr: Sah [24], Kur [23, 46].

Discelium Brid. [Disceliaceae]

- nudum (Dicks.) Brid.: Khm [88], Khs [109], Sah [24, 130].

Distichium Bruch, Schimp. \& W. Gümbel [Ditrichaceae]

- capillaceum (Hedw.) Bruch, Schimp. \& W. Gümbel: Am [7, 8, 32, 56, 130], Khm [41, 88], Khs [65, 109, 131], Prm [26, 28, 48, 130], Sah [24], Kur [21, 23, 25, 141].

- inclinatum (Hedw.) Bruch, Schimp. \& W. Gümbel: Am $[7,8,97]$

Ditrichum Timm ex Hampe [Ditrichaceae], see also Flexitrichum

- heteromallum (Hedw.) E. Britton: Am [56], Khm [88], Khs [65, 109], Prm [26, 42], Sah [24], Kur [21, 23, 25, 27, 141].

- lineare (Sw.) Lindb.: Prm [26, 28], Kur [23].

- macrorhynchum Broth.: Prm [45], Sah [24], Kur [23, 25].

- pallidum (Hedw.) Hampe: Am [130], Prm [26, 130], Kur [25].

- pusillum (Hedw.) Hampe: Am [32, 56, 130], Khs [40, 109], Prm [26, 71, 130], Kur [23].

- rhynchostegium Kindb.: Prm [28], Kur [23].

- zonatum (Brid.) Kindb.: Kur [113].

Dolichomitriopsis S. Okamura [Lembophyllaceae]

- diversiformis (Mitt.) Nog. (Isothecium diversiforme (Mitt.) Besch.): Prm [29, 131], Kur [23, 27].

Dozya Sande Lac. [Leucodontaceae]

- japonica Sande Lac.: Prm [26, 29, 37, 81], Kur [23, 81].

Drepanium C.E.O. Jensen [Amblystegiaceae]

- recurvatum (Lindb. \& Arnell) G. Roth (Hypnum recurvatum (Lindb. \& Arnell) Kindb.): Am [34], Khm [88], Prm [38, 133], Sah [24].

Drepanocladus (Müll. Hal.) G. Roth [Amblystegiaceae]

- aduncus (Hedw.) Warnst.: Am [7, 8, 32, 34, 43, 56, 70], Khm [41], Khs [40, 109, 163], Evr [39], Prm [26, 29, 38, 133], Sah [24, 36], Kur [21, 23, 25].

- polygamus (Schimp.) Hedenäs (Campylium polygamum (Schimp.) C.E.O. Jensen): Am [7, 8, 32], Prm [26, 29, 28], Sah [24, 36], Kur [23].

- sendtneri (Schimp. ex Müll. Hal.) Warnst.: Am [4, 7, 8], Prm [26, 28, 71].

Drummondia Hook. [Drummondiaceae]

- sinensis Müll. Hal.: Am [32, 56, 70], Khm [88], Khs [40, 123], Evr [39], Prm [26, 29],

Echinophyllum O'Brian [Thuidiaceae]

- sachalinense (Lindb.) O`Brian (Helodium sachalinense (Lindb.) Broth.): Khm [41, 88], Khs [40, 109, 123], Prm $[26,38,132]$, Sah $[24,36]$, Kur [23, 27].

Encalypta Hedw. [Encalyptaceae]

- brevicolla (Bruch \& Schimp.) Ångstr.: Khm [88].

- ciliata Hedw.: Am [8, 32, 56], Khm [41, 88], Khs [40, 65, 109], Prm [26, 28, 29, 42], Sah [24, 36], Kur [23].

- pilifera Funck: Am [58], Khs [109].

- procera Bruch: Am [97], Prm [97], Sah [24].

- rhaptocarpa Schwägr.: Sah [24, 60].

- trachymitria Ripart: Am [60]. 
Entodon Müll. Hal. [Entodontaceae]

- challengeri (Paris) Cardot (Entodon compressus (Hedw.) Müll. Hal.): Am [32, 133], Khm [41, 88], Khs [40, 163], Evr [39], Prm [26, 28, 29, 38, 71, 133].

- concinnus (De Not.) Paris: Am [8, 32, 56], Khm [88], Khs [40], Prm [26, 29, 133], Sah [24].

- diversinervis Cardot: Prm [26, 133] - (!!).

- flavescens (Hook.) A. Jaeger (Entodon rubicundus (Mitt.) A. Jaeger): Am [70], Khs [40], Evr [39], Prm [26, 28, 29, $38,48,71,133]$.

- giraldii Müll. Hal. (E. sinense (Dixon) Laz.): Am [56], Prm [26, 133].

- luridus (Griff.) A. Jaeger: Prm [26, 29, 133].

- scabridens Lindb.: Khs [40], Evr [39], Prm [26, 29, 38, 71, 133], Sah [24, 36], Kur [23].

- schleicheri (Bruch, Schimp. \& W. Gümbel) Demet.: Am [7, 8, 32, 56], Khm [88], Khs [40], Evr [39], Prm [26, 28, 29, 38, 42, 71], Sah [24].

- sullivantii (Müll. Hal) Lindb. (E. rufescens Laz.): Khm [145], Prm [26, 133] - (!!).

Entosthodon Schwägr. [Funariaceae]

- pulchellus (H. Philib.) Brugués (Funaria pulchella H. Philib.): Am $[8,32,56]$.

Ephemerum Hampe [Ephemeraceae]

- spinulosum Bruch \& Schimp.: Prm [94].

Eucladium Bruch, Schimp. \& W. Gümbel [Pottiaceae] - verticillatum (Brid.) Bruch, Schimp. \& W. Gümbel: Prm $[26,130]$

Eurhynchiadelphus Ignatov \& Huttunen [Brachytheciaceae] - eustegia (Besch.) Ignatov \& Huttunen (Eurbynchium eustegium (Besch.) Dixon): Am [7, 8, 32, 56], Khs [40, 109, 123], Evr [39], Prm [26, 28, 29, 38, 42, 48, 71, 132], Sah $[24,36]$, Kur [23].

Eurhynchiastrum Ignatov \& Huttunen [Brachytheciaceae] - pulchellum (Hedw.) Ignatov \& Huttunen (Eurbynchium pulchellum (Hedw.) Jenn.): Am [7, 8, 32, 35, 56, 70, 132], Khm [88], Khs [40, 65, 109, 123, 163], Evr [39], Prm [26, 28, 29, 38, 42, 48, 132], Sah [24, 36], Kur [23].

Eurohypnum Ando [Hypnaceae]

- leptothallum (Müll. Hal.) Ando: Am [56, 133], Khm [43, 88], Khs [40], Prm [26, 29, 28].

Fabronia Raddi [Fabroniaceae]

- ciliaris (Brid.) Brid.: Am [32, 56, 70, 115, 131], Khm [88, 115], Khs [109, 115, 131], Evr [39], Prm [26, 29, 38, 131, 115].

- rostrata Broth.: Am [56, 115].

Fauriella Besch. [Pylaisiadelphaceae]

- tenuis (Mitt.) Cardot: Khs [40], Prm [26, 28, 29, 71, 131], Sah [24, 36], Kur [23]

Filibryum W. Kim \& T. Yamag. [Taxiphyllaceae]

- ogatae (Broth. \& Yasuda) W. Kim \& T. Yamag. (Glossadelphus ogatae Broth. \& Yasuda): Kur [23].

Fissidens Hedw. [Fissidentaceae]

- adianthoides Hedw.: Prm [28], Sah [24, 36].

- beckettii Mitt.: Prm [87].

- bryoides Hedw.: Am [8, 32, 56, 70], Khm [88], Khs [40, 109, 123], Evr [39], Prm [26, 28, 29, 42, 130], Sah [24], Kur [25] - (!!).

- curvatus Hornsch.: Am [32], Khm [88], Kur [23] - (!!).

- dubius P. Beauv. (F. cristatus Wilson ex Mitt.): Am [70], Khm [MW], Khs [40], Evr [39], Prm [26, 28, 29, 38, 42, $48,71,130]$, Sah [24, 36], Kur [23, 25, 27, 141].
- gymnogynus Besch.: Evr [39], Prm [28, 71], Sah [24], Kur [23].

- byalinus Wilson \& Hook.: Prm [92, 94].

- marginatulus Meln.: Am [32] - (!!).

- neomagofukui Z. Iwats. \& Tad. Suzuki: Sah [24].

- nobilis Griff.: Kur [23, 25, 27].

- osmundoides Hedw.: Am [32, 70], Khm [88], Khs [109], Evr [39], Prm [26, 28, 29, 38, 71], Sah [24], Kur [23, 25] - (!!).

- taxifolius Hedw.: Am [32], Prm [26, 28, 29, 48, 130], Sah [24], Kur [23].

- teysmannianus Dozy \& Molk. (F. adelphinus Besch.): Kur [23, 27].

- viridulus (Sw.) Wahlenb.: Am [32], Sah [24] - (!!).

Flexitrichum Ignatov \& Fedosov [Flexitrichaceae]

- flexicaule (Schwägr.) Ignatov \& Fedosov (Ditrichum flexicaule (Schwägr.) Hampe): Khm [88], Khs [65], Prm [26], Sah [24] - (!!).

- gracile (Mitt.) Ignatov \& Fedosov (Ditrichum gracile (Mitt.) Kuntze): Khm [97], Prm [97], Sah [97]. Kur [97] - (!!).

Fontinalis Hedw. [Fontinalaceae]

- antipyretica Hedw.: Am [8, 32, 70], Khs [40, 109, 131], Prm [26, 28], Sah [24], Kur [23].

- antipyretica var. gracilis (Lindb.) Schimp.: Am [131], Prm [26, 29, 38].

- dalecarlica Bruch, Schimp. \& W. Gümbel: Am [34, 56].

- hypnoides Hartm.: Khs [131], Kur [23, 25].

- squamosa Hedw: Am [LE], Khs [LE], Prm [LE].

Forsstroemia Lindb. [Neckeraceae]

- cryphaeoides Cardot (Forsstroemia mandschurica Broth.): Prm [26, 28, 29, 71, 81, 131].

- goughiana (Mitt.) S. Olsson, Enroth \& D. Quandt (Neckera goughiana Mitt.): Prm [106].

- japonica (Besch.) Paris: Khs [123], Prm [26, 28, 29, 48, 81, 131], Sah [24, 36], Kur [23, 27, 81].

- stricta Laz.: Prm [28, 45, 81, 131] - (!!).

- trichomitria (Hedw.) Lindb.: Prm [26, 28, 29, 71, 81, 131].

- yezoana (Besch.) S. Olsson, Enroth \& D. Quandt (Neckera yezoana Besch.): Prm [26, 29, 106, 131], Sah [24, 36], Kur $[2,23,25,27]$.

Funaria Hedw. [Funariaceae]

- bygrometrica Hedw.: Am [7, 8, 32, 35, 56, 70], Khm [88], Khs [109], Evr [97], Prm [26, 28, 29, 42, 71], Sah [24, 36], Kur [21, 23, 25].

Glyphomitrium Brid. [Rhabdoweisiaceae]

- bumillimum (Mitt.) Cardot (G. warburgii auct.): Am [56, 70], Khm [41, 88], Khs [40, 65, 109], Evr [4, 39], Prm [26, 28, 29, 38, 42, 48, 131], Sah [24], Kur [23, 27] - (!!).

\section{Glossadelphus M. Fleisch. - see Filibryum}

Gollania Broth. [Pylaisiaceae]

- ruginosa (Mitt.) Broth. (G. amurensis Broth.): Am [32, 70, 133], Khm [88], Khs [123], Evr [39], Prm [26, 28, 29, 38, 42, 48, 71, 133].

- turgens (Müll. Hal.) Ando: Sah [24]. Kur [141].

Grimmia Hedw. [Grimmiaceae]

- alpestris (F. Weber \& D. Mohr) Schleich.: Sah [24]

- atrata Miel. ex Hornsch.: Kur [23].

- donniana Sm.: Khm [88], Khs [65], Prm [26] - (!!).

- elatior Bruch ex Bals.-Criv. \& De Not.: Am [7, 8, 103], Khm [88, 103], Prm [26, 103].

- elongata Kaulf.: Kur [23, 76]. 
- funalis (Schwägr.) Bruch, Schimp. \& W. Gümbel: Khm [88, 103], Prm [26, 103], Sah [24], Kur [97].

- hartmanii Schimp.: Khm [146a], Khs [109], Prm [97], Sah [24, 36], Kur [23, 103].

- incurva Schwägr.: Khm [88, 103], Prm [97].

- jacutica Ignatova, Bedn.-Ochyra, Afonina \& Muñoz: Am [8, 56], Khm [103], Khs [65, 103], Evr [39], Prm [103].

- longirostris Hook. (G. affinis Hornsch.): Am [7, 8, 32, 56], Khm [41, 88, 103], Khs [1, 65, 109, 163], Evr [39], Prm [38, 48, 103], Sah [24], Kur [23].

- mollis Bruch, Schimp. \& W. Gümbel: Khm [88, 103].

- mueblenbeckii Schimp.: Am [97], Khm [103], Khs [109].

- pilifera P. Beauv.: Am [7, 8, 32, 56, 70, 103], Khm [88, 103], Khs [40, 109], Evr [39], Prm [29, 28, 38, 71, 103].

- reflexidens Müll. Hal. (G. sessitana auct.): Khm [88, 103], Khs [65], Prm [97], Sah [24].

- tergestina Tomm. ex Bruch, Schimp. \& W. Gümbel: Prm [103], Sah [24].

- torquata Drumm.: Khm [103], Khs [65].

- unicolor Hook.: Khm [103].

Gymnostomum Nees \& Hornsch. [Pottiaceae]

- aeruginosum Sm.: Am [56], Khm [88], Prm [26, 28, 130], Sah [24], Kur [23, 25, 27$]$.

Hamatocaulis Hedenäs [Scorpidiaceae]

- vernicosus (Mitt.) Hedenäs (Drepanocladus vernicosus (Mitt.) Warnst.): Am [32, 34, 70, 132], Prm [26, 132], Sah [24].

Haplocladium (Müll. Hal.) Müll. Hal. [Thuidiaceae]

- angustifolium (Hampe \& Müll. Hal.) Broth.: Am [4, 32, 56], Khm [41, 88], Khs [40], Evr [39], Prm [26, 28, 29, $38,42,71,132]$.

- discolor (Broth. \& Paris) Broth.: Prm [59].

- micropbyllum (Hedw.) Broth.: Am [4, 32, 70], Khm [41, 88, 131], Khs [40, 131], Evr [4, 39], Prm [26, 28, 29, 38, 42, 71, 132], Sah [24, 36], Kur [23, 27].

- strictulum (Cardot) Reimers (H. fauriei auct.): Am [32, 70], Khs [40], Prm [26, 28, 29, 38, 132], Sah [24].

Haplohymenium Dozy \& Molk. [Anomodontaceae]

- flagelliforme L.I. Savicz: Evr [39], Prm [26, 28, 42, 132].

- longinerve (Broth.) Broth.: Khm [145], Prm [29, 28, 132, MHA].

- triste (Ces.) Kindb.: Am [32, 70], Khm [88], Khs [40, 109, 123], Evr [39], Prm [26, 28, 29, 38, 42, 48, 71, 132], Kur [23].

Hedwigia P. Beauv. [Hedwigiaceae]

- czernyadjevae Ignatova, Ignatov \& Fedosov: Khm [114].

- emodica Hampe ex Müll. Hal. var. emodica: Am [56, 114], Khm [114], Khs [114], Prm [114].

- emodica var. echinata Ignatova \& Ignatov: Am [114a], Khs [114a], Prm [114a], Kur [114a].

- kuzenevae Ignatova \& Ignatov: Am [114a, 56], Khm [114a].

- nemoralis Ignatova, Ignatov \& Fedosov: Prm [114].

Helodium Warnst. [Thuidiaceae]

- blandowii (F. Weber \& D. Mohr) Warnst.: Am [4, 7, 8, 32, 35, 56, 70, 132], Khm [41, 43], Khs [40, 109, 163], Evr [39], Prm [38, 132], Sah [24, 36], Kur [21].

- paludosum (Austin) Austin ex Broth.: Am [4, 7, 8, 32, 70, 132], Khs [40], Evr [4, 39], Prm [26, 28, 38, 132], Kur [23, 27].

Herpetineuron (Müll. Hal.) Cardot [Anomodontaceae] - toccoae (Sull. \& Lesq.) Cardot: Am [8, 32, 56, 70], Khm [145], Khs [40], Evr [39], Prm [26, 28, 29, 71, 132].

Herzogiella Broth. [Plagiotheciaceae]

- adscendens (Lindb.) Z. Iwats. \& W.B. Schofield (Campylium adscendens (Lindb.) Perss.): Khs [131], Prm [26, 28, 29, 38],
Sah [24, 36], Kur [21, 23, 25, 27, 141].

- striatella (Brid.) Z. Iwats. (Plagiothecium striatellum (Brid.) Lindb.): Khs [131], Prm [133] - (!!)(?).

- turfacea (Lindb.) Z. Iwats.: Am [8, 32, 56, 70, 133], Khm [41, 88], Khs [40, 109, 123, 131, 163], Prm [26, 28, 29, 38 48, 133], Sah [24, 36], Kur [21, 23, 25].

Heterocladium Bruch, Schimp. \& W. Gümbel [Heterocladiaceae]

- dimorphum (Brid.) Bruch, Schimp. \& W. Gümbel: Am [37, 43], Khm [37], Sah [24].

Heterophyllium (Schimp.) Kindb. [Pylaisiadelphaceae]

- affine (Hook.) M. Fleisch. (H. nemorosum (W.D.J. Koch ex Brid.) Kindb.): Am [32, 56, 70], Khm [41, 88], Khs [40, 65], Evr [39], Prm [26, 28, 38, 42, 48, 133].

Homalia Brid. [Neckeraceae]

- trichomanoides (Hedw.) Bruch, Schimp. \& W. Gümbel (H. japonica Besch.): Am [4, 7, 8, 32, 35, 56, 70, 131], Khm [41, 88], Khs [40, 65, 109, 123, 131], Evr [39, 131], Prm [26, 28, 29, 38, 42, 48, 71, 131], Sah [24, 36], Kur $[23,25,27]$.

Homaliadelphus Dixon \& P. de la Varde [Miyabeaceae] - targionianus (Mitt.) Dixon \&P. de la Varde (H. laevidentatus (S. Okamura) Nog.): Prm [26].

Homalothecium Bruch, Schimp. \& W. Gümbel [Brachytheciaceae]

- laevisetum Sande Lac.: Prm [26, 28, 29, 42, 48, 71, 132], Sah [24, 36], Kur [23, 25, 27].

Homomallium (Schimp.) Loeske [Pylaisiaceae]

- connexum (Cardot) Broth.: Am [32, 56], Khm [88], Prm $[26,29]$.

- incurvatum (Schrad. ex Brid.) Loeske: Am [7, 8, 56], Khs [40], Prm [26, 28, 133].

- japonico-adnatum (Broth.) Broth.: Prm [72, MHA].

- plagiangium (Müll. Hal.) Broth.: Prm [72, 133, MHA].

Hondaella Dixon \& Sakurai [Taxiphyllaceae]

- caperata (Mitt.) Ando, B.C. Tan \& Z. Iwats. (H. brachytheciella (Broth. \& Paris) Ando): Am [56], Khs [40], Evr [39], Prm [26, 133].

Hookeria Sm. [Hookeriaceae]

- acutifolia Hook. \& Grev.: Kur [VLA, NSK]

Hydrogonium (Müll. Hal.) A. Jaeger [Pottiaceae]

- amplexifolium (Mitt.) P.C. Chen (Barbula amplexifolia (Mitt.) A. Jaeger): Am [56].

- consanguineum (Thwaites \& Mitt.) Hilp. var. kurilensis (Ignatov \& Ignatova) Jan Kučera (Barbula indica (Hook.) Spreng. var. kurilensis Ignatov \& Ignatova): Kur [23, 82]. - gregarium (Mitt.) Jan Kučera: Prm [59].

Hygroamblystegium Loeske [Amblystegiaceae]

- humile (P. Beauv.) Vanderp. Goffinet \& Hedenäs (Leptodictyum kochii (Schimp.) Warnst.): Am [32, 123], Khs [123], Prm [26, 28, 29, 132], Sah [24, 36], Kur [23, 27$]$.

- tenax (Hedw.) Jenn.: Am [8], Prm [26, 28, 29, 38], Kur [23].

- varium (Hedw.) Mönk. (Amblystegium varium (Hedw.) Lindb.): Prm [26, 28, 132], Sah [24], Kur [21].

Hygrohypnella Ignatov \& Ignatova [Scorpidiaceae]

- bestii (Renauld \& Bryhn) Ignatov \& Ignatova: Kur [21, $23,25,141]$.

- ochracea (Turner ex Wilson) Ignatov \& Ignatova (Hygrobypnum ochraceum (Turner ex Wilson) Loeske): Am [7, 8, 32, 52, 56, 132], Khm [52, 88], Khs [40, 65, 109, 123], Prm [26, 28, 29, 42, 52], Sah [24, 36], Kur [21, 23, 25, 141]. 
- polaris (Lindb.) Ignatov \& Ignatova (Hygrohypnum polare (Lindb.) Loeske): Âm [4, 7, 8, 52, 132, 32, 56], Khm [52, 88], Khs [65, 123], Sah [24].

Hygrohypnum Lindb. [Amblystegiaceae]

- luridum (Hedw.) Jenn.: Am [34, 52, 56, 132], Prm [121].

Hylocomiastrum Broth. [Hylocomiaceae]

- pyrenaicum (Spruce) M. Fleisch. (Hylocomium pyrenaicum (Spruce) Lindb.): Am [43], Khm [43, 88], Khs [40, 65, 123], Prm [26, 48, 133], Sah [24, 36], Kur [21, 23, 25$].$

- umbratum (Hedw.) M. Fleisch.: Khs [1], Prm [144], Sah [36], Kur [23].

Hylocomiopsis Cardot [Hylocomiaceae]

- ovicarpa (Besch.) Cardot: Prm [1, 26, 48, 132], Sah [24, 36], Kur [23].

Hylocomium Bruch et al. [Hylocomiaceae]

- splendens (Hedw.) Bruch, Schimp. \& W. Gümbel: Am [4, $7,8,32,34,35,56,70]$, Khm $[41,43,88]$, Khs [40, 65, 109, 123, 163], Evr [39], Prm [26, 28, 29, 38, 42, 48], Sah $[24,36]$, Kur [21, 23, 25, 27, 141].

Hymenoloma Ochyra [Rhabdoweisiaceae]

- crispulum (Hedw.) Ochyra (Dicranoweisia crispula (Hedw.) Milde): Am [7, 8], Khm [88], Khs [65, 131], Prm [MW], Sah [24], Kur [21, 23, 25].

Hymenostylium Brid. [Pottiaceae]

- recurvirostrum (Hedw.) Dixon: Am [56], Khm [88], Prm [26, 29], Sah [24]. Kur [23].

Hyophila Brid. [Pottiaceae]

- involuta (Hook.) A. Jaeger: Am [56], Prm [26, MHA].

Hypnum Hedw. [Hypnaceae]

- cupressiforme Hedw: Am [7, 8, 32, 56, 133], Khm [41, 88], Khs [40, 65, 109, 123], Evr [39], Prm [26, 28, 29, 38, 42, 48, 71, 133], Sah [24, 36], Kur [23, 25, 27].

- cupressiforme var. subjulaceum Molendo: Am [56], Khm [88], Prm [133].

- fujiyamae (Broth.) Paris: Kur [23].

- saitoi Ando: Am [6, 8, 56], Khm [88].

Hypopterygium Brid. [Hypopterygiaceae]

- flavolimbatum Müll. Hal. (H. japonicum Mitt.): Am [70], Khs [40], Evr [39], Prm [26, 28, 38, 42, 48, 71, 131], Sah [24], Kur [23].

Isopterygiopsis Z. Iwats. [Plagiotheciaceae]

- alpicola (Lindb. \& Arnell) Hedenäs: Am [8, 32, 56], Khm [88], Khs [109], Kur [23].

- muelleriana (Schimp.) Z. Iwats.: Am [32, 34, 56, 133], Khm [41, 88], Khs [40, 65, 109, 123, 163], Evr [39], Prm $[26,28,29,38,71]$, Sah [24, 36], Kur [23, 25].

- pulchella (Hedw.) Z. Iwats.: Am [8, 32, 56, 133], Khm [88], Khs [65, 109], Prm [38, 133], Sah [24], Kur [21, 23].

Isothecium Brid. [Lembophyllaceae]

- hakkodense Besch.: Sah [24], Kur [23, 27].

Iwatsukiella W.R. Buck \& H.A. Crum [Leskeaceae]

- leucotricha (Mitt.) W.R. Buck \& H.A. Crum (Habrodon leucotrichus (Mitt.) Perss.): Am [2, 7, 8, 35, 43, 56], Khm [41, 88], Khs [65, 109, 163], Prm [26, 38, 42, 48, 131], Sah $[24,36]$, Kur [23, 25, 27].

Kiaeria I. Hagen [Rhabdoweisiaceae]

- blyttii (Bruch, Schimp. \& W. Gümbel) Broth.: Khs [65], Kur [23, 25].

- falcata (Hedw.) I. Hagen: Khs [65], Kur [21, 23, 25].

- glacialis (Berggr.) I. Hagen: Kur [21].
- starkei (F. Weber \& D. Mohr) I. Hagen: Khm [88], Khs [65], Sah [24], Kur [21, 23, 25].

Leptobryum (Bruch, Schimp. \& W. Gümbel) Wilson [Meesiaceae]

- pyriforme (Hedw.) Wilson: Am [8, 32, 56], Khs [40, 109, 131], Prm [131], Sah [24, 36], Kur [23, 27].

Leptodictyum (Schimp.) Warnst. [Amblystegiaceae]

- riparium (Hedw.) Warnst.: Khm [41], Khs [40, 123], Prm [26, 28, 42, 131], Sah [24, 36] - (!!).

Leptodontium (Müll. Hal.) Lindb. [Pottiaceae]

- flexifolium (Dicks.) Hampe: Am [32, 56, 70], Evr [39], Prm [26] - (!!).

Lescuraea Bruch, Schimp. \& W. Gümbel [Pseudoleskeaceae]

- incurvata (Hedw.) E. Lawton: Khm [88], Prm [72, MW], Sah [24].

- mutabilis (Brid.) Lindb.: Khs [123] - (!!)(?).

- patens Lindb.: Sah [24].

- radicosa (Mitt.) Mönk.: Sah [24].

- saxicola (Bruch, Schimp. \& W. Gümbel) Molendo: Khs [65, 109, 123], Prm [26, 38], Sah [24, 36], Kur [21, 23, 25].

Leskea Hedw. [Leskeaceae]

- polycarpa Hedw:: Am [32], Khm [41, 88, 131], Khs [131], Prm [26, 38, 131], Sah [24, 36], Kur [21, 23, 27].

Leucobryum Hampe [Leucobryaceae]

- glaucum (Hedw.) Ångstr.: Prm [26, 29, 38, 71, 130], Sah $[24,36]$.

- juniperoideum (Brid.) Müll. Hal.: Prm [26, 38], Sah [24], Kur [23, 25, 46, 128, 141].

Leucodon Schwägr. [Leucodontaceae]

- coreensis Cardot: Am [70, 81, 56], Khm [81], Evr [39], Prm [38, 48, 81].

- pendulus Lindb.: Am [4, 7, 8, 70, 81, 32, 56, 131], Khm $[41,81,88,131]$, Khs [40, 65, 109, 123, 131], Evr [4, 39, 81; 131], Prm [26, 28, 29, 38, 42, 48, 71, 81, 131], Sah [24].

- sciuroides (Hedw.) Schwägr.: Am [7, 8, 35, 56], Khm [41], Khs [40, 109], Evr [131], Prm [26, 28, 29, 42, 71, 131], Sah [24, 81], Kur [23, 27].

Lewinskya F. Lara, Garilleti \& Goffinet [Orthotrichaceae] - cf. affinis (Brid.) F. Lara, Garilleti \& Goffinet (Ortotrichum affine Brid.): Am [56] - (!!)

- elegans (Schwägr. ex Hook.\& Grev.) F. Lara, Garilleti \& Goffinet (reported as Orthotrichum speciosum Nees): Am [8, 34, 35, 56, 131], Khm [41, 88], Khs [109, 131], Prm [28, 131] - (!!).

- iwatsukii (Ignatov) F. Lara, Garilleti \& Goffinet (Orthotrichum iwatsukii Ignatov): Am [57], Khm [99], Khs [109].

- rupestris (Schleich. ex Schwägr.) F. Lara, Garilleti \& Goffinet (Orthotrichum rupestre Schleich. ex Schwägr.): Prm [26] - (!!).

- sordida (Sull. \& Lesq.) F.Lara, Garilleti \& Goffinet (Orthotrichum sordidum Sull. \& Lesq.): Am [8, 32, 56, 70], Khm $[41,88]$, Khs [40, 109, 123, 163], Evr [39], Prm [26, 28, 29, 38, 42, 48, 71], Sah [24, 36], Kur [21, 23, 27].

- transcaucasica Eckstein, Garilleti \& F. Lara: Am [56, 66a], Khm [66a]. Prm [IRK] - (!!), see L. striata.

Limnohypnum Ignatov \& Czernyadjeva [Climaciaceae] - mizushimae (Sak.) Ignatov \& Czernyadjeva (Leptodictyum mizushimae (Sakurai) Kanda): Kur [23, 95].

Lindbergia Kindb. [Leskeaceae]

- geniculata (Laz.) Ignatova \& Ignatov (Mamillariella geniculata Laz.): Prm [26, 28, 29, 108, 131].

- sinensis (Müll. Hal.) Broth.: Prm [26, 108] - (!!).

Loeskeobryum M. Fleisch. ex Broth. [Hylocomiaceae] 
- brevirostre (Brid.) M. Fleisch.: Sah [24, 36].

Loeskypnum H.K.G. Paul [Calliergonaceae]

- badium (Hartm.) H.K.G. Paul (Drepanocladus badius (Hartm.) G. Roth): Am [34, 43], Khm [43, 88], Khs [65], Sah [24], Kur [25].

- wickesii (Grout) Tuom.: Kur [25].

Lyellia R. Br. [Polytrichaceae]

- aspera (I. Hagen \& C.E.O. Jensen) Frye: Khm [117].

Macrocoma (Hornsch ex Müll. Hal.) Grout [Orthotrichaceae]

- tenus (Hook. \& Grev.) Vitt subsp. sullivantii (Müll. Hal.) Grout (Macromitrium bymenostomum Mont.): Prm [26, 28, 131, 99].

Macromitrium Brid. [Orthotrichaceae], see also Macrocoma - japonicum Dozy \& Molk.: Evr [39], Prm [26, 28, 29, 71, 131], Kur [IRK] - (!!).

Meesia Hedw. [Meesiaceae]

- longiseta Hedw. - Am [34], Prm [131].

- triquetra (Jolycl.) Ångstr. (M. trifaria H.A. Crum, Steere \& L.E. Anderson): Am [4, 7, 8, 32, 70, 131], Khm [99], Khs [99], Prm [26, 131], Sah [24], Kur [127].

- uliginosa Hedw.: Am [34, 131], Prm [131], Sah [24], Kur [23].

Meteorium (Brid) Dozy \& Molk. [Meteoriaceae]

- buchananii (Brid.) Broth.: Prm [29, 136].

Mielichboferia Nees \& Hornsch. [Mielichhoferiaceae]

- asiatica Tubanova \& Ignatova: Sah [99, 158].

- japonica Besch.: Kur [23, 25, 99, 141, 158] - (!!).

- mielichboferiana (Funck) Loeske: Prm [38] - (!!).

Miyabea Broth. [Miyabeaceae]

- fruticella (Mitt.) Broth.: Prm [26].

- rotundifolia Cardot: Prm [26, 132] - (!!)(?).

Mnium Hedw. [Mniaceae]

- heterophyllum (Hook.) Schwägr.: Am [32], Khs [109], Prm [26, 28, 29, 38, 71, 131], Sah [24], Kur [23, 27].

- lycopodioides Schwägr. (M. ambigunm Müll. Hal., M. laevinerve Cardot): Am [8, 32, 56], Khm [88], Khs [65, 109, 123, 131], Prm [26, 28, 38, 71], Sah [24, 36], Kur [23, 25] - (!!).

- marginatum (Dicks.) P. Beauv.: Am [7, 8, 32, 56], Khm $[43,88]$, Khs [65, 109], Evr [39], Prm [26], Sah [24, 36].

- orientale R.E. Wyatt, Odrzykoski \& T.J. Kop.: Khs [99], Prm [131], Sah [24], Kur [23, 27, 141] - (!!).

- spinosum (Voit) Schwägr.: Khm [88], Khs [65, 109], Prm [38, 48, 131].

- spinulosum Bruch, Schimp. \& W. Gümbel: Am [8, 56, 70], Khm [41, 88], Khs [40, 123, 163], Prm [26, 38, 42, 131].

- stellare Hedw.: Am [32], Khm [41], Khs [109, 123, 131], Evr [39], Prm [26, 29], Sah [24], Kur [21, 23, 25].

- thomsonii Schimp. (M. orthorrhynchum auct.): Am [7, 8, 32, 56, 70, 131], Khm [88], Khs [40, 163], Evr [39], Prm [26, 28, 29, 38, 42, 48, 131], Sah [24], Kur [21, 23, 25, 141].

Molendoa Lindb. [Pottiaceae]

- sendtneriana (Bruch, Schimp. \& W. Gümbel) Limpr.: Am [32, 56], Khm [88], Prm [26, 28, 29, 130].

Myrinia Schimp. [Amblystegiaceae] - pulvinata (Wahlenb.) Schimp.: Am [32], Sah [24].

Myurella Bruch, Schimp. \& W. Gümbel [Plagiothecieceae] - julacea (Schwägr.) Bruch, Schimp. \& W. Gümbel: Am [7, 8, 56], Khm [88], Khs [65], Sah [24], Kur [25].

- sibirica (Müll. Hal.) Reimers: Am [56], Khm [88], Prm
[26], Sah [24], Kur [23].

- tenerrima (Brid.) Lindb.: Am [32, 131], Sah [24] - (!!).

Myuroclada Besch. [Brachytheciaceae]

- longiramea (Müll. Hal.) Min Li,Y.F. Wang, Ignatov \& Huttunen (Brybnia brachycladula Cardot): Am [32, 56, 96], Khs [96], Prm [96], Sah [96], Kur [25].

- maximowiczii (G.G. Borshch.) Steere \& W.B. Schofield: Am [4, 7, 8, 32, 34, 35, 56, 70, 132], Khm [41, 88], Khs [40, 109, 131], Evr [39], Prm [26, 28, 29, 38, 42, 48, 71, 132], Sah [24, 36], Kur [23, 27].

Neckera Hedw. [Neckeraceae] see also Forsstroemia

- borealis Nog.: Khm [145], Prm [26, 42, 106], Sah [2, 24, 36], Kur [23, 27].

- konoi Broth. ex Cardot: Prm [106].

- oligocarpa Bruch (N. pennata var. tenera Müll. Hal.): Khm [145], Khs [40, 109].

- pennata Hedw.: Am [4, 7, 8, 32, 35, 56, 70, 131], Khm $[41,88]$, Khs [65, 109, 123, 131, 163], Evr [39], Prm [26, 28, 29, 38, 42, 48, 71, 106, 131], Sah [24, 36], Kur [23].

- polyclada Müll. Hal.: Prm [106].

Neckeropsis Reichardt [Neckeraceae]

- nitidula (Mitt.) M. Fleisch.: Sah [153] - (!!).

Niphotrichum (Bedn.-Ochyra) Bedn.-Ochyra \& Ochyra [Grimmiaceae]

- canescens (Hedw.) Bedn.-Ochyra \& Ochyra (Racomitrium canescens (Hedw.) Brid.): Am [7, 8, 35, 56, 70], Khm [41, 43, 88], Khs [65], Evr [39], Prm [26, 28, 29, 38, 71], Sah [24], Kur [21, 23, 27].

- elongatum (Ehrh. ex Frisvoll) Bedn.-Ochyra \& Ochyra: Kur [97].

- ericoides (Brid.) Bedn.-Ochyra \& Ochyra (Racomitrium ericoides (Brid.) Brid.): Am [97], Khm [43], Prm [97], Kur [23].

- japonicum (Dozy \& Molk.) Bedn.-Ochyra \& Ochyra (Racomitrium japonicum Dozy \& Molk.): Am [97, 32], Evr [39], Prm [28], Sah [24], Kur [128].

- muticum (Kindb.) Bedn.-Ochyra \& Ochyra: Kur [21, 23, 141].

- panschii (Müll. Hal.) Bedn.-Ochyra \& Ochyra (Racomitrium panschii (Müll. Hal.) Kindb.): Am [32, 56, 97], Khm [88], Khs [65]. Prm [97], Kur [97].

Nyholmiella Holmen \& E. Warncke [Orthotrichaceae]

- obtusifolia (Brid.) Holmen \& Warncke (Orthotrichum obtusifolium Brid.): Am [8, 32, 56, 70], Khm [88], Khs [109, 123, 131], Prm [26, 38], Sah [24], Kur [23].

Oedipodium Schwägr. [Oedipodiaceae]

- griffithianum (Dicks.) Schwägr.: Khs [65], Prm [91].

Okamuraea Broth. [Brachytheciaceae]

- brachydictyon (Cardot) Nog.: Am [70], Khs [40, 123], Evr [39], Prm [26, 28, 29, 42, 133], Kur [23].

- hakoniensis (Mitt.) Broth.: Evr [39], Prm [26, 28, 29, 38, 71], Sah [24, 36], Kur [23].

Oligotrichum DC. [Polytrichaceae]

- aligerum Mitt.: Khs [109], Prm [26], Sah [24], Kur [21, 23, 25].

- falcatum Steere: Khm [88, 118], Khs [65].

- hercynicum (Hedw.) Lam. \& DC.: Am [8, 43], Kur [21, 23, 25].

- parallelum (Mitt.) Kindb.: Khm [88], Khs [65, 123], Prm [26], Sah [24], Kur [21, 23, 25, 141].

Oncophorus (Brid.) Brid. [Rhabdoweisiaceae]

- crispifolius (Mitt.) Lindb.: Khm [145], Prm [26, 29, 48, 130].

- elongatus (I. Hagen) Hedenäs: Am [56], Khm [145] - (!!).

- virens (Hedw.) Brid.: Am [7, 8, 32, 130], Khm [145], Khs [40, 109], Prm [26, 38, 48, 130], Sah [24, 36], Kur [21].

- wablenbergii Brid.: Am [4, 7, 8, 32, 35, 56, 70, 130], 
Khm [41, 88], Khs [40, 65, 109, 123, 131, 163], Evr [4, 39], Prm [26, 28, 29, 38, 42, 71, 130], Sah [24, 36], Kur $[23,25,27,141]$.

Orthothecium Bruch, Schimp. \& W. Gümbel [Plagiotheciaceae]

- chryseon (Schwägr.) Bruch, Schimp. \& W. Gümbel: Khm [88], Sah [24].

- strictum Lorentz: Sah [24].

Orthotrichum Hedw. [Orthotrichaceae], see also Nyholmiella and Lewinskya

- anomalum Hedw.: Khs [131], Prm [29, 131], Sah [24].

- consobrinum Cardot: Prm [26, 28, 29, 131].

- rogeri Brid.: Am [32], Khm [145], Prm [MHA].

Oxyrrhynchium (Bruch, Schimp. \& W. Gümbel) Warnst. [Brachytheciaceae]

- savatieri (Schimp. ex Besch.) Broth.: Prm [26, 42], Kur [MHA] - (!!).

Oxystegus (Lindb. ex Limpr.) Hilp. [Pottiaceae]

- tenuirostris (Hook.\& Tayl.) A.J.E. Sm. (O. cylindricus (Bruch ex Brid.) Hilp.): Am [4, 32, 43, 56, 70, 130], Khm [41, 88], Khs [40, 65, 109], Evr [4, 39], Prm [26, 28, 29, $38,42,48,71,130]$, Sah $[24,36]$, Kur [21, 23, 25].

Paludella Brid. [Meesiaceae]

- squarrosa (Hedw.) Brid.: Am [8, 34, 56, 131], Khm [43], Khs [109], Prm [99], Sah [24], Kur [21].

Palustriella Ochyra [Amblystegiaceae]

- commutata (Hedw.) Ochyra (Cratoneuron commutatum (Hedw.) G. Roth): Am [7, 8, 132].

- decipiens (De Not.) Ochyra: Sah [24].

Paraleucobryum(Limpr.) Loeske [Dicranaceae]

- enerve (Thed.) Loeske: Am [8], Prm [26].

- longifolium (Hedw.) Loeske: Am [56], Khm [88], Khs [40, 109, 163], 39, Prm [26, 28, 29, 38, 130], Sah [24, 36], Kur [23].

Pelekium Mitt. [Thuidiaceae]

- pygmaeum (Bruch, Schimp. \& W. Gümbel) Touw (Thuidium pygmaeum Bruch, Schimp. \& W. Gümbel): Prm [5, 26, 28, 29, 42, 132], Kur [23, 27].

- versicolor (Müll. Hal.) Touw (Thuidium bipinnatulum Mitt., Cyrto-hypnum versicolor (Hornsch. ex Müll. Hal.) W.R. Buck \& H.A Crum): Khs [40], Prm [5, 26, 42, 132], Sah [5], Kur [125].

Philonotis Brid. [Bartramiaceae]

- americana Dism.: Sah [24, 124], Kur [23, 25, 113, 124].

- cf. capillaris Lindb.: Am [56] - (!!).

- fontana (Hedw.) Brid.: Am [43, 124, 32, 56], Khm [88], Khs [65, 109, 123], Erv [99], Prm [26, 29, 124, 131], Sah $[24,36,124]$, Kur $[21,23,25,27,124,141]$

- marchica (Hedw.) Brid.: Prm [131], Kur [124] - (!!).

- tomentella Molendo: Am [56, 57] - (!!).

- yezoana Besch. \& Cardot: Prm [124], Sah [24, 124], Kur $[21,23,25,124,141]$.

Physcomitrium (Brid.) Brid. [Funariaceae]

- acuminatum Bruch, Schimp. \& W. Gümbel (Physcomitrium eurystomum subsp. acuminatum (Bruch, Schimp. \& W. Gümbel) Giacom.): Prm [130, 26] - (!!).

- eurystomum Sendtn.: Am [97, 32]. Prm [26, 29].

- sphaericum (C.F. Ludw. ex Schkuhr) Brid.: Khs [131], Prm [97, MHA], Kur [25].

Plagiobryum Lindb. [Bryaceae] - (!!).

- bultenii (Ochi \& Perss.) Hedd.: Khm [88, 99], Sah [24, 99].
- japonicum Nog.: Prm [99], Kur [23, 27, 99].

Plagiomnium T.J. Kop. [Mniaceae]

- acutum (Lindb.) T.J. Kop. (Mnium acutum Lindb.): Am [4, 32, 34, 56, 70], Khm [41, 88], Khs [40, 109, 123, 131], Evr [39], Prm [26, 28, 29, 38, 42, 48, 71], Sah [24, 36], Kur $[21,23,25,27]$

- confertidens (Lindb. \& Arnell) T.J. Kop. (Mnium confertidens (Lindb. \& Arnell) Kindb.): Am [7, 8, 32, 35, 56, 70], Khm [41, 88], Khs [40, 65, 109, 123, 131], Evr [39], Prm [26, 28, 29, 38, 42, 48, 71], Sah [24].

- curvatulum (Lindb.) Schljakov: Am [99], Sah [24].

- cuspidatum (Hedw.) T.J. Kop. (Mnium cuspidatum Hedw.): Am [4, 7, 8, 32, 35, 56, 70], Khm [41, 88], Khs [40, 65, 109, 123, 131, 163], Evr [39], Prm [26, 28, 29, 38, 71], Sah $[24,36]$, Kur [23, 25].

- drummondii (Bruch \& Schimp.) T.J. Kop. (Mnium drummondii Bruch \& Schimp.): Am [32, 56], Khm [41, 88], Khs [40, 109, 131], Prm [26, 29, 48], Sah [24].

- ellipticum (Brid.) T.J. Kop. (Mnium rugicum Laurer): Am [4, 7, 8, 32, 56, 70], Khm [41, 88], Khs [40, 109, 123], Prm [26, 28, 29, 42, 71], Sah [24, 36], Kur [23, 25].

- japonicum (Lindb.) T.J. Kop.: Khs [123], Prm [28] - (!!).

- maximowiczii (Lindb.) T.J. Kop. (Mnium maximowiczii Lindb., Mnium micro-ovale Müll. Hal.): Am [43], Khm [88', Khs [40], Prm [26, 28, 29, 38, 42, 48], Kur [23].

- medium (Bruch \& Schimp.) T.J. Kop. (Mnium medium Bruch \& Schimp.): Am [34], Khm [41, 88], Khs [109, 123], Prm [26, 28, 29, 38], Sah [24, 36], Kur [21, 23, 25, 141].

- rostratum (Schrad.) T.J. Kop. (Mnium rostratum Schrad.): Am [7, 8, 56], Khm [MHA], Prm [26, 28, 71], Sah [24, 36].

- tezukae (Sak.) T.J. Kop.: Prm [99].

- vesicatum (Besch.) T.J. Kop. (Mnium vesicatum Besch.): Am [32], Prm [26, 28, 29, 42], Sah [24], Kur [23].

Plagiopus Brid. [Bartramiaceae]

- oederianus (Sw.) H.A. Crum \& L.E. Anderson: Am [32, 56, 131], Khm [41, 88], Khs [40, 65, 131], Prm [26, 29, 28], Sah [24], Kur [23, 25].

Plagiothecium Bruch, Schimp. \& W. Gümbel Plagiotheciaceae]

- cavifolium (Brid.) Z. Iwats. (incl. records of P. succulentum (Wilson) Lindb.): Am [32, 56, 70], Khm [41, 88], Khs [40, 65, 109], Evr [39], Prm [26, 28, 29, 48, 71], Sah [24, 36], Kur [21, 23, 25] - (!!).

- cordifolium Laz.: Prm [133] - (!!)(?).

- curvifolium Schlieph. ex Limpr.: Am [8], Khs [109], Prm [26], Kur [27].

- denticulatum (Hedw.) Bruch, Schimp. \& W. Gümbel: Am [7, 8, 32, 43, 56, 70], Khm [43, 88], Khs [65, 109, 123 , 131], Evr [39], Prm [26, 28, 29, 38, 42, 71], Sah [24, 36], Kur [21, 23, 25].

- euryphyllum (Cardot \& Ther.) Z. Iwats.: Khs [65, 109], Prm [26], Sah [24], Kur [23, 25].

- laetum Bruch, Schimp. \& W. Gümbel: Am [7, 8, 32, 43, 56], Khm [41, 88], Khs [109, 123], Evr [39], Prm [26, 28, 38, 42, 48], Sah [24, 36], Kur [23].

- latebricola Bruch, Schimp. \& W. Gümbel: Am [8, 32, 70], Khm [41], Khs [40, 109], Prm [1, 26, 28, 29, 42], Sah [24, 36], Kur [23, 25]

- nemorale (Mitt.) A. Jaeger: Am [8, 70], Khm [43, 88], Khs [40], Evr [39], Prm [26, 28, 29, 38, 42, 48, 71], Sah $[24,36]$, Kur [21, 23, 25, 27].

- obtusissimum Broth.: Sah [24, 36], Kur [23, 25, 27, 141]. - platycladum (Cardot) Broth.: Prm [133] - (!!)(?).

Platydictya Berk. [Plagiotheciaceae]

- fauriei (Cardot) Z. Iwats. \& Nog.: Sah [24].

- jungermannioides (Brid.) H.A. Crum (Amblystegiella jungermannioides (Brid.) Giacom.): Am [7, 8, 56], Kur [23, 25]. 
- minutissima (Sull. \& Lesq. ex Sull.) Crum (Amblystegium minutissimum (Sull.\&Lesq.ex Sull.) A. Jaeger): Sah [24].

Platygyrium Bruch, Schimp. \& W. Gümbel [Pylaisiadelphaceae]

- repens (Brid.) Bruch, Schimp. \& W. Gümbel: Am [7, 8, 32, 35, 56, 70], Khm [41, 88], Khs [109, 163], Evr [4, 39], Prm [26, 29, 38, 42, 71], Sah [24, 36], Kur [23, 25].

Platyhypnum Loeske (Ochyraea Váňa, Hygrohypnum p.p.) [Amblystegiaceae]

- alpestre (Hedw.) Ochyra (Ochyraea alpestris (Hedw.) Ignatov \& Ignatova): Khm [MW], Sah [24].

- cocbleariifolium (Venturi) Ochyra (Hygrohypnum cocbleariifolium (Venturi) Broth., Ochyraea cochleariifolia (Venturi) Ignatov \& Ignatova): Khm [52, 88], Sah [24].

- duriusculum (De Not.) Ochyra (Hygrohypnum duriusculum (De Not.) D.W. Jamieson, H. dilatatum (Wilson) Loeske, Ochyraea duriuscula (De Not.) Ignatov \& Ignatova): Khm [132], Khs [65, 123], Prm [26, 28, 42, 52, 71, 132], Sah [24], Kur [21, 23, 25, 52].

- norvegicum (Bruch, Schimp. \& W. Gümbel) Ochyra (Hygrohypnum norvegicum (Bruch, Schimp. \& W. Gümbel) J. Amann, Ochyraea norvegica (Bruch, Schimp. \& W. Gümbel) Ignatov \& Ignatova): Am [52], Khm [52, 88], Khs [109], Prm [MHA].

- smithii (Sw.) Ochyra (Ochyraea smithii (Sw.) Ignatov \& Ignatova): Kur [21].

Pleuridium Rabenh. [Ditrichaceae]

- subulatum (Hedw.) Rabenh.: Prm [130].

Pleuroziopsis Kindb. ex E. Britton [Climaciaceae]

- ruthenica (Weinm.) Kindb. ex E. Britton: Khm [41], Khs [40, 65, 109, 123, 163], Evr [39], Prm [26, 28, 29, 38, 42, 48], Sah [24, 36], Kur [23, 25, 27, 141].

Pleurozium Mitt. [Hylocomiaceae]

- schreberi (Brid.) Mitt.: Am [4, 7, 8, 32, 56], Khm [41, 43, 88], Khs [65, 109, 123, 163], Evr [39], Prm [26, 28, 29, 38 , 42], Sah [24, 36], Kur [21, 23, 25, 27, 141].

Podperaea Z. Iwats. \& Glime [Amblystegiaceae]

- krylovii (Podp.) Z.Iwats. \& Glime (Campylium krylovii (Podp.) Laz.): Am [8, 32, 37, 56], Khm [41, 88], Khs [40, 109], Prm [26, 28, 29, 42], Kur [23].

Pogonatum P. Beauv. [Polytrichaceae]

- contortum (Brid.) Lesq. (P. laterale (Brid.) Brid.): Khm [41], Khs [109, 123, 131], Evr [39], Prm [26, 28, 29, 38, 42, 48, 71], Sah [24, 36], Kur [23, 25, 27].

- dentatum (Brid.) Brid. (P. capillare (Michx.) Brid.): Am [7, 32, 56], Khm [43, 88], Khs [40, 65, 109, 163], Prm [26, 38], Sah [24, 36], Kur [21, 23, 25, 141].

- inflexum (Lindb.) Sande Lac.: Prm [26], Kur [23].

- japonicum Sull. \& Lesq.: Khm [S], Khs [40, 65, 109], Prm [26, 29, 38, 48, 71], Sah [24, 36], Kur [21, 23, 25].

- spinulosum Mitt.: Khs [97], Prm [26, 28, 29, 71], Kur $[23,27]$.

- urnigerum (Hedw.) P. Beauv.: Am [4, 7, 8, 32, 56], Khm $[43,88]$, Khs [40, 65, 109, 131, 163], Evr [39], Prm [26, $28,29,38,42,48]$, Sah [24, 36], Kur [21, 23, 25, 141].

Poblia Hedw. [Mielichhoferiaceae]

- andalusica (Höhn.) Broth.: Am [32, 56], Khs [99], Sah [24], Kur [25].

- andrewsii A.J. Shaw: Am [32, 56], Khm [88], Khs [65, 109], Prm [MHA], Kur [23].

- annotina (Hedw.) Lindb.: Am [56], Sah [24], Kur [23].

- bulbifera (Warnst.) Warnst.: Am [50, 32, 56], Khm [MHA], Khs [109], Kur [25].

- cruda (Hedw.) Lindb.: Am [7, 8, 32, 56, 70], Khm [43,
88], Khs [65, 109, 123, 131, 163], Prm [26, 28, 38], Sah [24], Kur [21, 23, 25, 141].

- crudoides (Sull. \& Lesq.) Broth.: Khm [88], Sah [24], Kur [23, 25, 141].

- drummondii (Müll. Hal.) A.L. Andrews: Am [32], Khm [88, 50], Khs [65], Sah [24], Kur [21, 23, 25].

- elongata Hedw.: Am [8, 32, 34, 56], Khm [88], Khs [40, 109, 131], Prm [28, 38], Kur [23, 25].

- elongata var. greenii (Brid.) A.J. Shaw (P. minor Schleich. ex Schwägr.): Âm [56], Khm [88], Prm [26].

- filum (Schimp.) Mårtensson: Am [56], Khs [109], Prm [99], Sah [99], Kur [23, 25, 141].

- lescuriana (Sull.) Ochi: Am [32, 56].

- longicollis (Hedw.) Lindb.: Am [56, 131], Khm [41, 88], Khs [109], Evr [39], Prm [26, 38], Sah [24], Kur [141].

- ludwigii (Spreng. ex Schwägr.) Broth.: Khs [65], Prm [99].

- lutescens (Limpr.) H. Lindb.: Am [31, 32], Khs [31, 109].

- melanodon (Brid.) A.J. Shaw: Khs [109], Sah [24, 36], Kur [23].

- nutans (Hedw.) Lindb.: Am [7, 8, 32, 56, 70], Khm [41, 43, 88], Khs [40, 65, 109, 123, 131, 163], Evr [39], Prm [26, 38], Sah [24, 36], Kur [21, 23, 25, 141].

- proligera (Kindb.) Lindb. ex Broth.: Am [32, 56], Khm [88], Khs [109], Evr [39], Prm [26, 50], Sah [24], Kur $[23,25]$.

- sphagnicola (Bruch, Schimp. \& W. Gümbel) Broth.: Sah (Cherdantseva, unpubl.) - (!!).

- tundrae A.J. Shaw: Am [56], Khs [109], Prm [MHA], Sah [NSK], Kur [23, 25].

- vexans (Limpr.) H. Lindb.: Am [32 (cf.)], Khs [65].

- wablenbergii (F. Weber \& D. Mohr) A.L. Andrews: Am [32], Khm [88], Khs [65, 109, 123, 163], Prm [131, MHA], Sah [24, 36], Kur [21, 23, 25, 141].

Polytrichastrum G.L. Sm. [Polytrichaceae], see also Polytrichum

- alpinum (Hedw.) G.L. Sm. (Polytrichum alpinum Hedw.): Am [7, 8, 32, 56, 119], Khm [43, 88], Khs [40, 65, 109 , 123, 131, 163], Evr [39], Prm [26, 29, 38, 48, 119], Sah [24, 36, 119], Kur [21, 23, 25, 119, 141].

- fragile (Bryhn) Schljakov: Khm [43].

- septentrionale (Brid.) E.I. Ivanova, N.E. Bell \& Ignatov: Sah $[24,119]$.

- sexangulare (Flörke ex Brid.) G.L. Sm.: Khm [88, 119], Sah [24, 119], Kur [21, 23, 25, 119].

- sphaerothecium (Besch.) J.-P. Frahm: Kur [21, 23, 25, 46, 119].

Polytrichum Hedw. [Polytrichaceae], see also Polytrichastrum - commune Hedw.: Am [4, 7, 8, 32, 35, 56], Khm [41, 88], Khs [40, 65, 109, 131, 163], Evr [39], Prm [26, 29, 38, 42, 71], Sah [24, 36], Kur [21, 23, 25, 27, 141].

- densifolium Wilson ex Mitt. (Polytrichastrum formosum (Hedw.) G.L. Sm. var. densifolium (Wilson ex Mitt.) Osada): Evr [39], Prm [38, 120], Sah [135], Kur [21, 23, 120] - (!!). - byperboreum R. Br.: Am [56, 57], Khs [65], Sah [97].

- jensenii I. Hagen: Am [7, 8, 56, 70], Khm [43], Evr [39], Prm [38], Sah [24], Kur [21, 23, 27].

- juniperinum Hedw.: Am [4, 7, 8, 32, 35, 56, 70], Khm $[41,43]$, Khs [40, 65, 109, 123, 163], Evr [39], Prm [26, 28, 29, 38, 42, 48, 71], Sah [24, 36], Kur [21, 23, 25, 141].

- longisetum Sw. ex Brid. (P. gracile Menzies): Am [7, 8, 32, 35, 56, 119], Khm [41, 43], Khs [109, 123], Prm [26, 28, 38, 119], Sah [24, 36], Kur [21, 23, 25, 141].

- pallidisetum Funck (P. obioense auct.): Am [7, 8, 35, 70], Khm [41], Khs [40, 109, 120], Prm [1, 26, 28, 38, 42, 119], Sah [24, 36, 120], Kur [23, 25, 120, 141].

- piliferum Hedw: Am [7, 8, 32, 35, 56, 70], Khm [41, 43, 88], Khs [109, 123, 131], Prm [26, 29, 38], Sah [24, 36], 
Kur [21, 23, 25].

- strictum Brid. (P. alpestre Hoppe): Am [4, 7, 8, 32, 35, 56, 70], Khm [41, 43, 88], Khs [40, 109, 123], Prm [26, 29, 38], Sah [24, 36], Kur [21, 23, 25].

- swartzii Hartm.: Am [7, 8, 32, 56], Sah [24, 36].

Pseudobryum (Kindb) T.J.Kop. [Mniaceae]

- cinclidioides (Huebener) T.J. Kop.: Am [7, 8, 32, 56], Khm [41, 88, 131], Khs [109, 123, 131], Evr [39], Prm [LE], Sah [24, 36], Kur [21, 23, 25].

Pseudocalliergon (Limpr.) Loeske [Amblystegiaceae]

- trifarium (F. Weber \& D. Mohr) Loeske (Calliergon trifarium (F. Weber \& D. Mohr) Kindb.): Prm [132].

Pseudohygrohypnum Kanda [Pylaisiaceae]

- eugyrium (Bruch, Schimp. \& W. Gümbel) Kanda (Hygrobypnum engyrium (Bruch, Schimp. \& W. Gümbel) Loeske): Prm [26, 52, MHA].

- subeugyrium (Renauld \& Cardot) Ignatov \& Ignatova (Hygrohypnum subeugyrium (Renauld \& Cardot) Broth.: Khm [52, 88], Khs [65], Prm [29, 52], Kur [23, 25, 52].

Pseudoleskeella Kindb. [Pseudoleskeellaceae]

- catenulata (Brid. ex Schrad.) Kindb.: Prm [26, 28], Sah $[24,36]$.

- nervosa (Brid.) Nyholm (Leskeella nervosa (Brid.) Loeske): Khm [88], Khs [163], Prm [26], Sah [24, 36], Kur [23].

- papillosa (Lindb.) Kindb.: Am [56], Khm [88], Khs [65, 109].

- rupestris (Berggr.) Hedenäs \& L.Söderstr: Sah [24], Kur [25].

- tectorum (Funck ex Brid.) Kindb. ex Broth.: Am [8], Khm [88], Khs [65, 109], Prm [26, 28], Sah [24], Kur [25].

Pseudoleskeopsis Broth. [Leskeaceae]

- zippelii (Dozy \& Molk.) Broth.: Prm [30].

Pseudotaxiphyllum Z. Iwatz. [Plagiotheciaceae]

- elegans (Brid.) Z. Iwats.: Khs [65], Prm [38].

- pobliaecarpum (Sull. \& Lesq.) Z. Iwats.: Sah [156], Kur $[23,25,113]$.

Psilopilum Brid. [Polytrichaceae]

- cavifolium (Wilson) I. Hagen: Am [56].

- laevigatum (Wahlenb.) Lindb.: Kur [141].

Pterigynandrum Hedw. [Pterigynandraceae]

- filiforme Hedw.: Prm [26, MHA], Sah [24], Kur [23].

Ptilium De Not. [Pylaisiaceae]

- crista-castrensis (Hedw.) De Not.: Am [4, 7, 8, 32, 35, 56, 70], Khm [41, 88], Khs [40, 65, 109, 123, 131, 163], Evr [39], Prm [26, 28, 29, 38, 42, 48], Sah [24, 36], Kur $[21,23,25,27]$.

Ptychomitrium Fürnr. [Ptychomitriaceae]

- sinense (Mitt.) A. Jaeger: Am [ 56, 57], Khm [97], Khs [40], Prm [26, 28, 29, 71].

Pylaisia Bruch, Schimp. \& W. Gümbel [Pylaisiaceae]

- brotheri Besch.: Khm [43], Prm [26, 28, 29, 38], Kur $[23,27]$.

- curviramea Dixon: Am [7, 43], Khm [88], Sah [24, 36] - (!!).

- falcata Bruch, Schimp. \& W. Gümbel: Am [56, 57], Prm [MHA], Sah [24], Kur [23].

- obtusa Lindb.: Sah [24, 36], Kur [23].

- polyantha (Hedw.) Bruch, Schimp. \& W. Gümbel: Am 77, 8, 35, 32, 43, 56], Khm [41, 88], Khs [40, 65, 109, 123 , 163], Evr [39], Prm [26, 28, 29, 38, 42, 71], Sah [24, 36], Kur [23].

- selwynii Kindb.: Am [32, 56, 70], Khm [41, 88], Khs [109, 163], Evr [39], Prm [26, 28, 29, 38, 71], Sah [24,
36], Kur [25].

- steerei (Ando \& Higuchi) Ignatov: Am [32, 56], Khm [MHA], Khs [109].

- stereodontoides Broth. \& Yasuda ex Ihsiba (see also note to P. intricata (Hedw.) Bruch, Schimp. \& W. Gümbel) Khs [131], Evr [39], Prm [26, 29, 38].

- subcircinata Cardot: Prm [26, 48], Sah [24, 36], Kur [23].

Pylaisiadelpha Cardot [Pylaisiadelphaceae]

- tenuirostris (Bruch \& Schimp. ex Sull.) W.R. Buck: Am [8, 15, 32, 56, 70], Khm [15, 41, 88], Khs [40, 109], Evr [39], Prm [15, 26, 28, 29, 38, 42, 48, 71], Sah [24], Kur [23].

- tristoviridis (Broth.) Afonina, Tsubota \& Ignatova: Khs [65], Prm [15].

Racomitrium Brid. [Grimmiaceae]

- lanuginosum (Hedw.) Brid.: Am [34], Khm [43, 88], Khs [65], Prm [26, 38, 48], Sah [24, 36], Kur [21, 23, 25, 141].

Rauiella Reimers [Thuidiaceae]

- fujisana (Paris) Reimers: Am [4, 32, 70], Khm [41, 88], Khs [40, 109, 123, 163], Evr [4, 39], Prm [26, 28, 29, 38, 42, 48], Sah [24, 36], Kur [23, 25, 27$]$.

Rhabdoweisia Bruch, Schimp. \& W. Gümbel [Rhabdoweisiaceae]

- crispata (Dicks. ex With.) Lindb. (Rhabdoweisia kuzenevae Broth.): Am [8, 32, 56], Khm [41, 88], Khs [40, 65, 109], 39, Prm [26, 29, 38, 42, 48], Sah [24, 36], Kur [23, 25].

Rhizomnium (Broth.) T.J.Kop. [Mniaceae]

- andrewsianum (Steere) T.J. Kop.: Khm [88].

- magnifolium (Horik.) T.J. Kop.: Khm [41, 88], Khs [65, 109, 123, 163], Prm [38, 48], Sah [24, 36], Kur [23, 25, 27, 141].

- nudum (E. Britton \& R.S. Williams) T.J. Kop.: Khs [65, 109], Sah [24], Kur [21, 23, 25, 141].

- parvulum (Mitt.) T.J. Kop. (Mnium minutulum Besch.): Prm [26, 28, 29, 48].

- pseudopunctatum (Bruch \& Schimp.) T.J. Kop.: Kur [21, $23,25]-(! !)$.

- striatulum (Mitt.) T.J. Kop. (Mnium striatulum Mitt.): Am [32, 70], Khm [88], Khs [65, 109, 123], Prm [26, 28, 29, $38,42,71]$, Sah [24], Kur [23, 25].

- tuomikoskii T.J. Kop.: Am [32], Khm [MW], Prm [99], Sah [24], Kur [23]

Rhodobryum (Schimp.) Limpr. [Bryaceae]

- ontariense (Kindb.) Kindb.: Am [32, 56, 57, 70], Khm [41], Prm [29, MHA], Sah [24], Kur [23].

- roseum (Hedw.) Limpr.: Khm [88], Khs [40, 109] - (!!).

Rhynchostegium Bruch, Schimp. \& W. Gümbel [Brachytheciaceae]

- aquaticum A. Jaeger (R. muelleri A. Jaeger): Prm [26, 48], Sah [24], Kur [23, 25] - (!!), see note for R. riparioides.

- pallidifolium (Mitt.) A. Jaeger: Khs [VLA, MHA], Prm [VLA, MHA], Sah [24], Kur [23].

- rotundifolium (Scop. ex Brid.) Bruch, Schimp. \& W. Gümbel: Sah [24, 36].

Rhytidiadelphus (Limpr.) Warnst. [Hylocomiaceae]

- japonicus (Reimers) T.J. Kop.: Khs [65], Prm [26, 29], Sah [24], Kur [23, 25, 27, 141$]$.

- squarrosus (Hedw.) Warnst: Sah [24, 36], Kur [21, 23, 25, 141].

- subpinnatus (Lindb.) T.J. Kop.: Am [43], Khm [43, 88], Khs [109], Prm [MHA], Sah [24, 36], Kur [23, 27, 141].

- triquetrus (Hedw.) Warnst.: Am [4, 7, 8, 32, 35, 56, 70], Khm [41, 43, 88], Khs [40, 65, 109, 123, 163], Evr [39], Prm [26, 28, 29, 38, 42, 48], Sah [24, 36], Kur [23, 25, 27].

Rhytidium (Sull.) Kindb. [Rhytidiaceae] 
- rugosum (Hedw.) Kindb.: Am [4, 7, 8, 32, 35, 43, 56], Khm [41, 43, 88], Khs [40, 65, 109, 123, 163], Evr [39], Prm [26, 28, 29, 38, 48], Sah [24, 36], Kur [23].

Rigodiadelphus Dixon [Pseudoleskeaceae]

- robustus (Lindb.) Nog.: Prm [26, MHA], Sah [24, 36], Kur [21, 23, 25, 27].

Saelania Lindb. [Ditrichaceae]

- glaucescens (Hedw.) Broth.: Am [4, 7, 8, 32, 35, 56], Khm $[41,88]$, Khs [40, 65, 109, 123], Prm [26, 38, 48], Sah [24, 36], Kur [23].

Sanionia Loeske [Scorpidiaceae]

- uncinata (Hedw.) Loeske (Drepanocladus uncinatus (Hedw.) Warnst.): Am [4, 7, 8, 32, 35, 56, 70], Khm [41, 43, 88], Khs [40, 65, 109, 123, 163], Evr [39], Prm [26, 28, 29, 38, 42, 48], Sah [24, 36], Kur [21, 23, 25, 27, 141].

Sasaokaea Broth. [Leskeaceae]

- aomoriensis (Paris) Kanda: Khs [126]; Prm [72].

Schistidium Bruch, Schimp. \& W. Gümbel [Grimmiaceae] - agassizii Sull. \& Lesq.: Khm [88].

- austrosibiricum Ignatova \& H.H. Blom: Am [56, 112].

- bakalinii Ignatova \& H.H. Blom: Sah [24], Kur [25, 107$].$

- crenatum H.H. Blom: Khs [65].

- elegantulum H.H. Blom: Prm [33].

- flaccidum (De Not.) Ochyra: Sah [24].

- frigidum H.H. Blom: Khm [33].

- konoi (Broth.) Ignatova \& H.H. Blom: Prm [97], Kur [97].

- lancifolium (Kindb.) H.H. Blom: Am [32, 56, 97], Khm [97], Khs [65, 109], Prm [97], Sah [24], Kur [23, 25].

- liliputanum (Müll. Hal.) Deguchi: Am [56], Khm [88], Prm [97], Sah [24], Kur [23].

- marginale H.H. Blom, Bedn.-Ochyra \& Ochyra: Am [56].

- maritimum (Sm. ex R. Scott) Bruch, Schimp. \& W. Gümbel: Sah [24], Kur [23, 25, 141].

- papillosum Culm. (S. strictum auct.): Am [8, 56], Khs [109], Prm [26, 28, 29, 38, 48], Sah [24], Kur [21, 23].

- platyphyllum (Mitt.) Perss. (S. alpicola (Hedw.) Limpr. var. alpicola): Am [7, 56], Prm [26, 48], Sah [24].

- pruinosum (Wilson ex Schimp.) G. Roth: Khs [109].

- pulchrum H.H. Blom: Am [32, 56, 97], Khm [97], Khs [109, 39], Prm [97], Sah [24], Kur [23].

- rivulare (Brid.) Podp. (S. alpicola (Hedw.) Limpr. var. rivulare (Brid.) Limpr.): Am [8, 32], Khm [41, 88], Khs [109, 163], Prm [28, 48], Sah [24], Kur [21, 23] - (!!).

- sibiricum Ignatova \& H.H. Blom: Am [32, 56, 107], Khs [109], Prm [107], Sah [97].

- sordidum I. Hagen: Khs [65].

- subjulaceum H.H. Blom: Am [56].

- tenuinerve Ignatova \& H.H. Blom: Sah [24], Kur [25].

- trichodon (Brid.) Poelt: Sah [24].

- trichodon var. nutans H.H. Blom: Khm [97], Sah [24].

Schistostega D. Mohr [Schitostegaceae]

- pennata (Hedw.) F.Weber \& D. Mohr: Am [8, 32, 34, 56], Khm [41, 88], Khs [40, 65, 109], Prm [26, 48], Sah [24], Kur $[23,27]$.

Schwetschkeopsis Broth. [Pylaisiadelphaceae]

- fabronia (Schwägr.) Broth.: Khs [40], Evr [39], Prm [26, $28,29,38,48]$.

Sciuro-hypnum (Hampe) Hampe [Brachytheciaceae]

- brotheri (Paris) Ignatov \& Huttunen: Sah [24, 36], Kur [23].

- curtum (Lindb.) Ignatov: Am [32, 56, 84], Khm [88], Khs [109, 123], Prm [84], Sah [24], Kur [23, 25].
- glaciale (Bruch, Schimp. \& W. Gümbel) Ignatov \& Huttunen: Sah [24], Kur [141].

- latifolium (Kindb.) Ignatov \& Huttunen: Khm [84, 88].

- plumosum (Hedw.) Ignatov \& Huttunen (Brachythecium plumosum (Hedw.) Bruch, Schimp. \& W. Gümbel): Am [8, 32, 56], Khm [84], Khs [40, 65, 109], Evr [39], Prm [26, 28, 29, 38, 48, 84], Sah [24, 36], Kur [21, 23, 25, 84].

- populeum (Hedw.) Ignatov \& Huttunen (Brachythecium populeum (Hedw.) Bruch, Schimp. \& W. Gümbel): Am [70], Khm [41, 84], Khs [40, 109, 163], Evr [39], Prm $[26,28,29,38,42,71,84]$, Sah [24, 36, 84], Kur [21, 23, $25,27,84]$.

- reflexum (Starke) Ignatov \& Huttunen (Brachythecium reflexum (Starke) Bruch, Schimp. \& W. Gümbel): Am [8, 32, 56], Khm [43, 88], Khs [65, 109], Prm [26, 28, 29, 38], Sah [24, 36], Kur [21, 23, 25, 27].

- starkei (Brid.) Ignatov \& Huttunen (Brachythecium starkei (Brid.) Bruch, Schimp. \& W. Gümbel): Khm [43], Khs [84, 109], Prm [26, 42, 84], Sah [24, 36], Kur [21, 23, 25, 141].

- uncinifolium (Broth. \& Paris) Ochyra \& Żarnowiec: Khs [109], Prm (VLA, MHA) Sah [24], Kur [21, 23, 25, 141].

Scopelophila (Mitt.) Lindb. [Pottiaceae]

- ligulata (Spruce) Spruce: Prm [26].

Scorpidium (Schimp.) Limpr. [Scorpidiaceae]

- cossonii (Schimp.) Hedenäs (Drepanocladus intermedius (Lindb.) Warnst.): Am [34], Sah [24], Kur [25].

- revolvens ( $\mathrm{Sw}$. ex anon.) Rubers (Drepanocladus revolvens (Sw.) Warnst.): Am [8, 34], Khm [43], Khs [65], Sah [24], Kur [21, 25].

- scorpioides (Hedw.) Limpr.: Kur [21, 23].

Scouleria Hook. [Scouleriaceae]

- pulcherrima Broth.: Am [32, 110], Khm [110].

Seligeria Bruch, Schimp. \& W. Gümbel [Seligeriaceae], see also Blindiadelphus

- calcarea (Hedw.) Bruch, Schimp. \& W. Gümbel: Prm [97], Sah [97].

- donniana (Sm.) Müll. Hal.: Am [58, 56], Prm [97].

- pusilla (Hedw.) Bruch, Schimp. \& W. Gümbel: Prm [97].

- tristichoides Kindb.: Am [56, 58], Prm [97], Kur [97].

Sphagnum L. [Sphagnaceae]

- alaskense R.E. Andrus \& Janssens: Am [56, 139], Khm [138], Khs [65, 163], Sah [24], Kur [23, 138].

- angustifolium (C.E.O. Jensen ex Russow) C.E.O. Jensen: Am [4, 7, 8, 32, 35, 56], Khm [41, 43, 88], Khs [109, 123], Evr [39], Prm [26, 38], Sah [24, 36], Kur [21].

- aongstroemii Hartm.: Am [8, 34, 43, 56], Khm [41, 43, 88], Khs [65], Sah [24], Kur [23].

- auriculatum Schimp.: Am [4].

- balticum (Russow) C.E.O. Jensen: Am [4, 7, 8, 43, 56], Khm [43, 88], Khs [40, 109], Prm [29], Sah [24, 36].

- beringiense A.J. Shaw, R.E. Andrus \& B. Shaw: Am [56, 138], Khm [138], Khs [65], Prm [138].

- capillifolium (Ehrh.) Hedw. (S. nemoreum Scop.): Am [34, 43, 56], Khm [43, 88], Khs [109, 123], Prm [26, 38], Sah $[24,36]$, Kur [21, 23, 25].

- centrale C.E.O. Jensen: Am [4, 7, 8, 32], Khs [40], Evr [39], Prm [26, 38], Sah [24, 36], Kur [21].

- compactum Lam. \& DC.: Am [7, 8, 35, 56], Khm [43, 88], Khs [65, 109, 123], Sah [24], Kur [21, 23, 25, 141].

- contortum Schultz: Am [7, 8, 32, 70], Khm [43], Evr [39], Prm [26, 38], Sah [24], Kur [21, 23, 141] - (!!).

- cuspidatum Ehrh. ex Hoffm.: Am [43], Sah [36].

- divinum Flatberg \& Hassel (S. magellanicum auct.): Am [4, 7, 8, 32, 35, 43, 56], Khm [41, 43, 88], Khs [40, 109, 123], Evr [39], Prm [26, 29, 38], Sah [24, 36]. Kur [23] - (!!). 
- fallax (H. Klinggr.) H. Klinggr.: Am [4, 8, 43, 56], Khm [43], Khs [40, 109, 123], Evr [39], Prm [72], Sah [24, 36], Kur $[21,23,25,127]$.

- fimbriatum Wilson: Am [7, 8, 32], Khm [41, 43, 88], Khs [109], Evr [39], Prm [26, 29], Sah [24, 36], Kur [21, 23 , 27, 127].

- flexuosum Dozy \& Molk.: Am [32, 56, 70], Khm [88], Khs [40, 109], Prm [26, 38], Sah [24], Kur [23].

- fuscum (Schimp.) H. Klinggr.: Am [4, 7, 8, 32, 35, 43, 56], Khm [43, 88], Khs [40], Evr [39], Prm [26, 38], Sah [24, 36], Kur [23, 25].

- girgensohnii Russow: Am [4, 7, 8, 32, 35, 43, 56], Khm [41, 43, 88], Khs [40, 65, 109, 123], Evr [39], Prm [26, 29 38, 42, 48], Sah [24, 36], Kur [21, 23, 25, 141].

- imbricatum Hornsch. ex Russow: Am [4, 32, 34, 43, 56 137, 139], Evr [39, 137], Prm [26, 29, 137], Kur [23].

- inexpectatum Flatberg: Prm [IRK], Kur [25].

- inundatum Russow: Khs [109], Sah [24, 36], Kur [23].

- jensenii H. Lindb.: Am [34, 56], Khm [88], Sah [24, 36], Kur [23].

- lenense H. Lindb. ex L.I. Savicz: Am [7, 8, 43, 56], Khm $[43,88]$, Khs [123], Prm [38], Sah [24, 36].

- lindbergii Schimp.: Am [34, 56], Khm [43, 88], Sah [24, 36], Kur [21].

- majus (Russow) C.E.O. Jensen: Am [69], Sah [24].

- mirum Flatberg \& Thingsgaard: Am [56].

- miyabeanum Warnst.: Kur [25].

- obtusum Warnst.: Am [4, 7, 8, 32, 56, 69], Khm [88], Khs [109], Prm [26, 29, 38], Sah [24, 36], Kur [23].

- orientale L.I. Savicz: Am [4, 7, 8, 32, 69], Khs [40], Prm $[26,28$. IRK $]$.

- palustre L.: Am [43], Khm [41, 43, 88], Prm [26, 28], Sah $[24,36]$, Kur [23, 25, 127]

- papillosum Lindb.: Am [34], Sah [24, 36], Kur [21, 23, $25,128]$.

- perfoliatum L.I. Savicz: Am [56], Kur [23].

- platyphyllum (Lindb. ex Braithw.) Warnst.: Am [34], Prm [29], Sah [24, 36], Kur [25].

- pulchrum (Lindb. ex Braithw.) Warnst.: Kur [23, 25, 128].

- pungifolium X.J. Li: Sah [24].

- quinquefarium (Lindb. ex Braithw.) Warnst.: Sah [24], Kur [21, 141]

- riparium Ångstr.: Am [34, 32, 43, 56], Khm [43, 88], Khs [109], Sah [24, 36], Kur [21, 23, 141].

- rubellum Wilson: Am [7, 8, 56], Khs [40, 109], Sah [24, 36], Kur [23].

- rubiginosum Flatberg: Khs [11, 109, 163], Sah [24].

- russowii Warnst.: Am [7, 8, 32, 43, 56], Khm [43, 88], Khs [109, 123, 163], Prm [38], Sah [24, 36], Kur [21, 23, 128].

- squarrosum Crome: Am [4, 7, 8, 32, 35, 56, 70], Khm $[41,43,88]$, Khs [40, 65, 109, 123], Evr [39], Prm [26, 28, 29, 38, 71], Sah [24, 36], Kur [21, 23, 25, 27, 141].

- steerei R.E. Andrus: Sah [24].

- subfulvum Sjörs: Am [32], Kur [23, 25].

- subnitens Russow \& Warnst.: Am [34].

- subsecundum Nees: Am [32, 34], Khm [MHA], Prm [26, 29, 38], Sah [24, 36], Kur [21, 23, 25].

- subtile (Russow) Warnst.: Kur [23].

- tenellum (Brid.) Pers. ex Brid.: Am [56], Sah [24, 36], Kur [21, 25, 128].

- teres (Schimp.) Ångstr.: Am [4, 7, 8, 32, 43, 56, 70], Khm [41, 88], Khs [109], Evr [39], Prm [38], Sah [24, 36], Kur $[21,23,25,27]$.

- tundrae Flatberg: Am [56, 57], Khs [109].

- warnstorfii Russow: Am [4, 7, 8, 34, 32, 43], Khm [43, 88], Khs [65, 109], Sah [24, 36], Kur [21, 23, 25, 27$].$

- wulfianum Girg.: Am [8, 43], Prm [38], Sah [24, 36].
Splachnum Hedw. [Splachnaceae]

- ampullaceum Hedw.: Am [35, 130], Khs [109].

- luteum Hedw.: Am [MW], Khm [99].

- rubrum Hedw: Am [8], Khm [43, 88], Khs [131], Prm [38].

- sphaericum Hedw.: Khm [88].

Stereodon (Brid.) Mitt. [Pylaisiaceae]

- bambergeri (Schimp.) Lindb.: Khs [163], Sah [24] - (!!).

- calcicola (Ando) Afonina \& Cherd.: Am [56], Prm [13].

- callichrous (Brid.) Lindb. var. japonicus Ando: Am [35], Khm [43, 88], Prm [26, 133], Kur [141] - (!!).

- densirameus (Ando) Afonina \& Ignatova (Hypnum demsirameum Ando): Am [32], Khs [13], Prm [13, 42], Kur [13, 23].

- dieckei (Renauld \& Cardot) Broth.: Kur [13, 23, 25].

- fauriei (Cardot) Afonina (Hypnum fauriei Cardot): Am [8, 13, 32, 56], Khm [13, 88], Khs [163], Evr [39], Prm [13, $26,28,38,42,48]$.

- hamulosus (Bruch, Schimp. \& W. Gümbel) Lindb.: Khm [88], Kur [MHA] - (!!).

- holmenii (Ando) Ignatov \& Ignatova: Am [32], Khm [9], Khs [65], Kur [23, MHA].

- pallescens (Hedw.) Mitt. (Hypnum pallescens (Hedw.) P. Beauv.): Am [8, 32, 56], Khm [88], Khs [40, 109, 123, 163], Evr [4, 39], Prm [26, 28, 29, 38, 42, 71], Sah [24, 36], Kur [21, 23, 25].

- plicatulus Lindb. (Hypnum plicatulum (Limdb.) A. Jaeger): Am [9, 32, 34, 56, 70], Khm [41, 43, 88], Khs [65, 109, 123], Evr [39], Prm [26, 29, 38, 48], Sah [24, 36], Kur [9, $21,23,25,27,141]$.

- plumiformis (Wilson) Mitt. (Hypnum plumiforme Wilson): Prm [13, 26, 29, 37], Kur [23, 127].

- procerrimus (Molendo) Bauer: Sah [24].

- subimponens (Lesq.) Broth. var. subimponens: Am [56], Prm [9], Kur [23, MHA].

- subimponens var. ulophyllum (Müll. Hal.) Afonina: Khm [9].

- vaucheri (Lesq.) Lindb. ex Broth. (Hypnum vaucheri Lesq.): Am [56], Prm [26, 29, 28], Sah [24].

Straminergon Hedenäs [Calliergonaceae]

- stramineum (Dicks. ex Brid.) Hedenäs (Calliergon stramineum (Dicks. ex Brid.) Kindb.): Am [4, 7, 8, 32, 43, 56], Khm [43, 88], Khs [109, 123], Prm [29, 38], Sah [24, 36], Kur $[21,23,25,141]$.

Streblotrichum P. Beauv.

- convolutum (Hedw.) P. Beauv. (Barbula convoluta Hedw.): Prm [26, 28, 130], Sah [24].

Struckia Müll. Hal. [Plagiotheciaceae]

- enervis (Broth.) Ignatov, T.J. Kop.\& D.G. Long: Khm [59].

Symblepharis Mont.[Dicranaceae]

- vaginata (Hook.) Wijk \& Margad.: Prm [16].

Syntrichia Brid. [Pottiaceae]

- amphidiacea (Müll. Hal.) R.H. Zander: Prm [14].

- laevipila Brid. (Tortula laevipila (Brid.) Schwägr.): Am [32, MHA], Khm [145], Khs [109], Prm [26, 28] - (!!).

- norvegica F. Weber: Sah [24], Kur [21].

- pagorum Milde: Am [56], Khm [MW] - (!!).

- ruralis (Hedw.) F. Weber \& D. Mohr (Tortula ruralis (Hedw.) G. Gaertn., B. Mey. \& Scherb.): Khs [131], Sah [24], Kur [21].

- sinensis (Müll. Hal.) Ochyra (Tortula sinensis (Müll. Hal.) Broth.): Am [18, 56], Prm [18, 26, 29, 28].

Taxiphyllum M. Fleisch. [Taxiphyllaceae]

- alternans (Cardot) Z. Iwats.: Prm [26].

- aomoriense (Besch.) Z. Iwats.: Am [70], Khs [40], Evr 
[39], Prm [26, 28, 29, 38, 42, 48, 71], Sah [24, 36], Kur $[23,27]$.

- taxiphylloides (Ando \& Higuchi) Higuchi: Prm [75].

- taxirameum (Mitt.) M. Fleisch.: Am [70], Khs [40, 131], Prm [26, 28, 42, 48, 71], Kur [25].

Tayloria Hook. [Splachnaceae]

- lingulata (Dicks.) Lindb.: Sah [24].

Tetraphis Hedw. [Tetraphidaceae]

- geniculata Girg. ex Milde: Khm [41], Khs [40, 65, 109, 123, 131], Prm [26, 28, 29, 38], Sah [24, 36], Kur [23, 27].

- pellucida Hedw.: Am [4, 7, 8, 32, 56, 70], Khm [41, 43, 88], Khs [40, 109, 123, 131, 163], Evr [4, 39], Prm [26, $28,29,38,42,48,71]$, Sah [24, 36], Kur [23].

Tetraplodon Bruch, Schimp. \& W. Gümbel [Splachnaceae] - angustatus (Hedw.) Bruch, Schimp. \& W. Gümbel: Am [7, 8, 43, 56], Khm [41, 88], Khs [109, 123], Prm [130], Sah [24, 36], Kur [23].

- mnioides (Hedw.) Bruch, Schimp. \& W. Gümbel: Am [8, 34, 35], Khm [88], Khs [65, 123], Prm [26, 38, 130], Sah [24], Kur [21, 23, 141].

Tetrodontium Schwägr. [Tetraphidaceae]

- ovatum (Funck) Schwägr. (T. brownianum var. ovatum (Funck) Wijk \& Margad.): Khm [97].

- repandum (Funck) Schwägr.: Khm [88], Sah [156], Kur $[23,25,46]$.

Thamnobryum Nieuwl. [Neckeraceae]

- coreanum (Cardot) Nog. \& Z. Iwats.: Prm [102], Sah [24].

- neckeroides (Hook.) E. Lawton (T. obtusatum (Lindb. \& Arnell) Bard. \& Cherd., T. vorobjovii (Laz.) Ochyra): Khs [109], Prm [26, 28, 29, 38, 42, 48, 102], Sah [24], Kur $[23,25,102]$.

- subseriatum (Mitt. ex Sande Lac.) B.C. Tan (Thamnobryum sandei (Besch.) Z. Iwats.): Prm [28, 102], Kur [23].

- subserratum (Hook.ex Harv.) Nog. \& Z. Iwats.: Prm [102], Kur [23, 102].

Thuidium Bruch, Schimp. \& W. Gümbel [Thuidiaceae]

- assimile (Mitt.) A. Jaeger (T. philibertii Limpr.): Am [4, 7, 8, 32, 35, 56, 70], Khm [41, 88], Khs [40, 65, 109, 123, 131, 163], Evr [4, 39], Prm [26, 28, 29, 38, 42, 48, 71], Sah [24, 36], Kur [23].

- cymbifolium (Dozy \& Molk.) Dozy \& Molk.: Khs [40], Prm [26, 28, 29, 42, 71], Kur [113].

- delicatulum (Hedw.) Bruch, Schimp. \& W. Gümbel: Am [7, 8, 34, 132], Khm [132], Sah [24], Kur [23, 25, 27] - (!!).

- glaucinoides Broth.: Prm [72], Kur [23, 25].

- kanedae Sakurai: Prm [5, 26], Kur [23, 27].

- pristocalyx (Müll. Hal.) A. Jaeger (Thuidium glancinum (Mitt.) Bosch \& Sande Lac.): Am [5], Khs [40], Evr [39], Prm [26, 29, 38], Kur [23].

- subglaucinum Cardot: Prm [45], Kur [113].

- submicropteris Cardot: Khs [40], Evr [39], Prm [5, 26, 28, 29, 48], Kur [5, 23, 27].

- tamariscinum (Hedw.) Bruch, Schimp. \& W. Gümbel: Sah [24, 36], Kur [23, 25, 27].

Timmia Hedw. [Timmiaceae]

- bavarica Hessl.: Am [8, 56], Sah [24].

- comata Lindb. \& Arnell: Am [56], Sah [24].

- megapolitana Hedw.: Am [32, 56 ], Khm [88], Prm [26, 28], Sah [24].

- norvegica J.E. Zetterst.: Khm [88], Kur [27, 23].

Timmiella (De Not.) Limpr. [Pottiaceae]

- anomala (Bruch \& Schimp.) Limpr.: Am [4, 56], Prm [26].
Tomentypnum Loeske [Amblystegiaceae]

- nitens (Hedw.) Loeske: Am [4, 7, 8, 32, 35, 56, 70], Khm [43], Khs [40, 109], Evr [39], Prm [132], Sah [24], Kur [21].

Tortella (Müll. Hal.) Limpr. [Pottiaceae]

- alpicola Dixon: Sah [24].

- fragilis (Hook.\& Wilson) Limpr.: Am [7, 8, 56], Khs [109], Sah [24, 36], Kur [23, 25].

- inclinata (R. Hedw.) Limpr.: Prm [130].

- tortuosa (Hedw.) Limpr.: Am [7, 8, 32, 56], Khm [88], Prm [MHA], Sah [24], Kur [23, 25].

Tortula Hedw. [Pottiaceae]

- acaulon R.H. Zander: Prm [MHA].

- cernua (Huebener) Lindb.: Prm [146]. Kur [23].

- edentula Ignatova \& Ignatov: Kur [23, 82].

- hoppeana (Schultz) Ochyra: Khm [88], Sah [24].

- mucronifolia Schwägr:: Khs [130], Prm [26, 130], Sah [24].

- truncata (Hedw.) Mitt. (Pottia truncata (Hedw.) Bruch \& Schimp.): Prm [26, 28].

- ucrainica (Laz.) R.H. Zander (Desmatodon ucrainicus Laz.): Prm [130] - (!!).

Trachycystis Lindb. [Mniaceae]

- flagellaris (Sull. \& Lesq.) Lindb.: Khm [41], Khs [40, 123, 131], Prm [26, 28, 29, 38, 42, 48, 71], Sah [24, 36], Kur [21, 23, 25, 27, 141].

- microphylla (Dozy \& Molk.) Lindb.: Khs [40], Prm [26, 29], Sah [24].

- ussuriensis (Maack \& Regel) T.J. Kop. (Mnium ussuriense Maack \& Regel, Mnium immarginatum Broth.): Am [4, 7, 8, 32, 56, 70], Khm [41, 43, 88], Khs [40, 131], Evr [39], Prm [26, 28, 29, 38, 42, 48], Sah [24, 36], Kur [21, 23].

Trematodon Michx. [Bruchiaceae]

- ambiguus (Hedw.) Hornsch.: Khm [145], Prm [26], Sah [24], Kur [23].

- longicollis Michx.: Khm [88], Sah [24], Kur [23, 113].

Trichodon Schimp.

- cylindricus (Hedw.) Schimp. (Ditrichum cylindricum (Hedw.) Grout): Am [8, 32, 56], Khm [41, 88], Khs [109], Evr [39], Prm [26, 29], Kur [23].

Trichostomum Bruch [Pottiaceae]

- crispulum Bruch: Am [7, 8, 32, 56], Prm [26, 28], Kur [23].

Ulota D. Mohr [Orthotrichaceae]

- crispula Bruch: Khm [99], Khs [62], Sah [62], Kur [62] - (!!).

- crispa (Hedw.) Brid.: Am [32], Prm [26, 28, 29, 42, 48, 62].

- curvifolia (Wahlenb.) Sw: Am [8, 34], Khm [88], Khs [131].

- drummondii (Hook.\& Grev.) Brid.: Prm [26], Sah [24, 36, 62], Kur [21, 23, 62, 141].

- intermedia Schimp.: Khm [62], Khs [62], Prm [62], Sah [62], Kur [62].

- japonica (Sull. \& Lesq.) Mitt.: Khs [99], Prm [99, 62], Sah [24, 36, 62], Kur [23, 62].

- orientalis Fedosov \& Ignatova: Khs [62], Sah [99], Kur [IRK].

- pacifica Fedosov \& Ignatova: Sah [99], Kur [62].

- rehmannii Jur.: Am [56, 62], Khm [62], Khs [62], Prm [62], Sah [62], Kur [62].

- reptans Mitt.: Am [56, 99] Khm [88, 62], Khs [109, 62], Sah $[24,62]-(! !)$.

Venturiella Müll. Hal. [Erpodiaceae]

- sinensis (Venturi) Müll. Hal.: Prm [66].

Vesicularia (Müll. Hal.) Müll. Hal. [Pylaisiaceae] 
- flaccida (Sull. \& Lesq.) Z. Iwats.: Prm [26, 29, 28].

Warnstorfia Loeske [Calliergonaceae]

- exannulata (Bruch, Schimp. \& W. Gümbel) Loeske (Drepanocladus exannulatus (Bruch, Schimp. \& W. Gümbel) Warnst.): Am [4, 8, 32, 34, 56], Khm [43, 88], Khs [40, 65, 109], Prm [26, 28], Sah [24, 36], Kur [21, 23].

- fluitans (Hedw.) Loeske (Drepanocladus fluitans (Hedw.) Warnst.): Am [4, 34, 43, 56], Khm [41, 43], Khs [40], Evr [39], Prm [38], Sah [24, 36], Kur [21, 23, 25, 27, 141].

- procera (Renauld \& Arnell) Tuom. (Drepanocladus procerus (Renauld \& Arnell) Warnst.): Am [132].

- pseudostraminea (Müll. Hal.) Tuom. \& T.J. Kop.: Sah [24], Kur [23, 128].

- sarmentosa (Wahlenb.) Hedenäs (Calliergon sarmentosum (Wahlenb.) Kindb., Sarmentypnum sarmentosum (Wahlenb.) Tuom. \& T.J. Kop.): Am [8, 43, 56], Khm [88], Khs [65], Kur [21, 25].

- trichophylla (Warnst.) Tuom. \& T.J.Kop.: Sah [104].

- tundrae (Arnell) Loeske - Khm [88].

Weissia Hedw. [Pottiaceae]

- brachycarpa (Nees \& Hornsch.) Jur.: Am [32, 34, 56], Khs [40, 109], Prm [26, MHA].

- controversa Hedw.: Am [32], Prm [26, 28, 29, 38], Sah [24], Kur [23, 25].

- edentula Mitt.: Khm [88].

- longifolia Mitt.: Prm [94].

- planifolia Dixon: Prm [26, 48] - (!!).

- rutilans (Hedw.) Lindb.: Am [32], Prm [26, 29, 28$]$

Zygodon Hook \& Taylor [Orthotrichaceae]

- sibiricus Ignatov, Ignatova, Z. Iwats. \& B.C. Tan: Am [8, 32, 56], Khm [41, 88], Khs [109], Prm [26, 29, 42], Sah [24] - (!!).

\section{Comments on species doubtful and erroneously} reported from at least some regions (species are given in the checklist with (!!))

Amblystegium radicale was reported for Primorsky Territory by Bardunov \& Cherdantseva [26], however no one specimen was confirmed in the course of the present revision, so its occurrence in RFE seems doubtful, though not impossible.

Amblystegium serpens var. juratzkanum is not separated from $A$. serpens by most authors.

Atrichum tenellum: according to Cherdantseva (unpubl.), records of Atrichum tenellum from Primorsky Territory [26, 27] are erroneous, however this mainly European species occurs in Asian Russia as a rare adventive plant.

Brachythecium campestre: earlier it was cited for many regions of the Russian Far East. In the refined sense of the species it seems to be rare in the region [93].

Brachythecium rutabulum: early records of Brachythecium rutabulum from Sakhalin after revision of the samples were relegated by Cherdantseva to B. rivulare. Records from Prm she also considered to be doubtful. Most likely, the Far Eastern plants belong to a separate species, while the B. rutabulum s. str. does not occur in the Russian Far East at all.

Brachythecium salebrosum: variation of this species in the Russian Far East exceeds what is known in other regions, and it is likely that so-called specimens represent another species, but until a special study will be conducted, there is no better choice than to retain this name for specimens which we leave All known reports of this species from the Russian Far East are doubtful and awaiting a special revision.
Bucklandiella sudetica was reported from Prm [72], however, we could not locate specimens in herbaria; the occurrence of this northern and high mountain species in Vladivostok area is highly unlikely.

Campylophyllopsis hispidulum (Campylidium hispidulum, Campylium hispidulum) was thought to be an American species by [73]. It remains unclear if $C$. hispidulum occurs in Asia, and if so, what are its distinctions from C. sommerfeltii, and what are their distribution ranges in Asia.

Campylopus fragilis: record from Prm [72] can not be checked as the specimen was lost. Previously this species was considered in broader sense.

Codriophorus acicularis (Racomitrium aciculare): records from Am [8], Khm [88] were erroneous [97].

Coscinodon cribrosus: the specimen from $\mathbf{K h m}$, Badzhal Range, lacks sporophytes, thus it was tentatively referred to C. cribrosus basing only on gametophyte traits, ecology and our knowledge about distribution of Coscinodon species in the Russian Far East.

Cynodontium polycarpon: all record from the Russian Far East are doubtful, as this species was earlier understood in a broader sense.

Dicranella subsecunda: status and distribution of this species are still poorly known.

Dicranum lorifolium: Lang \& Stech [128a] in the course of molecular phylogenetic analysis revealed separate position of Dicranum samples form Primorsky Territory, which form a well supported clade sister to $D$. japonicum. They referred these specimens to Dicranum cf. lorifolium Mitt. (described from Himalayas).

Entodon diversinervis: all specimens found in VLA which were previously referred to this species were reidentified as E. scabridens; however, we have not seen specimens of Lazarenko.

Flexitrichum gracile: for a long time this species has not been distinguished from F. flexicaule, so distribution of both species in Russian Far East needs in revision. The former species is more common in the area.

Fissidens osmundoides: before ca. 2000 this name was often erroneously applied to F. gymnogynus, e.g. in Prm [26, 29], so its distribution in the Russian Far East remains insufficiently known.

Forsstroemia stricta and $\boldsymbol{F}$. producta (Hornsch.) Paris comprise a taxonomic problem. Lazaranko [131] in original description and later Ignatov \& Cherdantseva [81] thought that the plants are dioicous, likely partly due to a relatively small collection of Forsstroemia stricta. However, recent collections provided evidence that the species is autoicous, and therefore almost totally fits the description of pantropical F. producta. At the same time, preliminary molecular data indicate possible difference between American (from where F. producta was described) and Afro-Asian population, thus we prefer reserve Forsstroemia stricta as a name for Asian plants.

Forsstroemia trichomitria: record from Am [7] is based on specimen later re-identified as Cryphaea amurensis.

Glyphomitrium humillimum (Mitt.) Cardot (G. warburgii auct.): we provide here the names under which it was cited in Russia, but its taxonomic identity remains unclear.

Grimmia donniana: records from Khm [88] are based on erroneous identifications, records from Prm [26] have to be revised.

Herzogiella striatella: no vouchers were found in the 
course of the present revision, we consider its occurrence in the Russian Far East as doubtful.

Leptodictyum riparium was reported from Am [8. 7], but no one specimen was found in herbaria.

Leptodontium styriacum (Jur.) Limpr. was recorded from Prm [26], but later its records were referred to $\boldsymbol{L}$. flexifolium [90]. However, this genus needs in a revision. It is likely that two species occur in Russia, including Russian Far East, but nomenclature of these species remains unclear.

Lescuraea mutabilis (Brid.) Lindb.: record from Khs [123] is probably erroneous and belongs to $L$. saxicola, which was included in L. mutabilis as a variety in some taxonomic treatments.

Lewinskya elegans is reported based on partly revised collections of L. speciosa, which does not occur eastward Ural [99].

Lewinskya rupestris (Orthotrichum rupestre): records from Prm [26] have to be confirmed.

Lewinskya striata: records from the Russian Far East belong to L. transcaucasica [99].

Lindbergia (incl. Mamillariella) in Russia was revised by Ignatova et al. [108], resulting in exclusion of L. brahcyptera, L. duthei and L. japonica from moss flora of Russia.

Macromitrium japonicum: records from Sah [24], Kur [23] were found to be erroneous [99], however later in IRK we found this species in Kur.

Mielichhoferia: revision by Tubanova et al. [158] found that records of $M$. japonica from Sah [24], belong to $M$. asiatica. Some unrevised records of $M$. mielichhoferiana from Prm [38], most likely also belong to $M$. asiatica.

Miyabea rotundifolia was reported from from Prm [26, $132]$, but all so-named specimens studied by us were found to be $M$. fruticella.

Mnium laevinerve was reported from Khs [40], Prm [26, 29, 42, 131]. T.J. Koponen synonymized it with M. lycopodioides [99].

Mnium orientale: all records of Mnium hornum from the Russian Far East belong to Mnium orientale [99].

Myurella tenerrima (Brid.) Lindb. was reported for Prm basing on [132] record for Kedrovaya Pad Reserve, which is likely an error; recent lists of this reserve [71, 75a] do not include this species.

Neckeropsis nitidula was reported from South Sakhalin by Sugawara [153]; it was not collected since then in the area and voucher specimens were not located. So this record seems to be doubtful.

Oncophorus is considered here according to the taxonomic revision by Hedenäs [74], though only few most recent collections were identified basing on his splitting concept, in particular, specimens from Badzhal Range (Khm) and Zeya State Reserve (Am). Distribution of O. wablenbergii s.str. (incl. O. compactus) and O. elongatus, as well as other species, which have not been found in considered area yet, need in further study.

Oxyrinchium savatieri: record from Prm [72] can not be confirmed, as the specimen was lost. This is a rare species, known in Russia only from southern Kuril Islands.

Philonotis capillaris was found to be composed of two different lineages in Russia [124]; one lineage from the Russian Far East, reported from Am [32] and Khs [109]. After additional study these specimens were re-identified [89].
However, plants with morphological characters of $P$. capillaris were later collected in Am [56]; taxonomy of this group needs an additional study.

Philonotis tomentella: records of this species from $\mathrm{Khm}$ [43] and Kur [21] were not confirmed by the recent revision [124].

Physcomitrium acuminatum remains a poorly known taxon, its occurrence in Russian Far East remains to be confirmed [97].

Plagiobryum: species delimitation and distribution was revised and changed considerably in $\Phi \mathrm{MP}-4$, with a number of reconsideration of species identity in various previous records.

Plagiomnium japonicum: record from Am [8] is not confirmed and likely erroneous.

Plagiothecium cavifolium according to our observations does not differ from P. succulentum (reported from Prm [26, $29,133]$ ), although the problem of species delimitation requires further studies.

Plagiothecium cordifolium remains insufficiently known species, regarding both its status and distribution.

Plagiothecium platycladum remains insufficiently known species, regarding both its status and distribution.

Plagiothecium succulentum: according to our observations, this species does not differ from $P$. cavifolium, although collections from the Russian Far East were not specially revised.

Poblia sphagnicola remains a poorly known species, requiring special revision.

Pylaisia curviramea: morphological differentiation of this species from $P$. polyantha is not very distinct, although molecular data support its independent status [19].

Rhizomnium pseudopunctatum: according to [95], records of this species from $\mathrm{Khm}$ and Sah belong to R. magnifolium.

Rhodobryum roseum was repeatedly reported instead of R. ontariense in old publications, e.g. in Am [7, 8, 35], before the latter species was assumed as a more common in many regions of Asian Russia. However, R. roseum is rare in the south and usually avoids calcareus substrates.

Sphagnum contortum: according to a preliminary revision of A.I. Maksimov [140], S. contortum does not occur in the Russian Far East. Majority of so called specimens belong to closely related $S$. beringiense.

Sphanum divinum Brid.: all specimens from the Russian Far East referred previously to $S$. magellanicum belong to this species, based on study of Hassel et al. [72a]; our revision of specimens from the study area confirms this conclusion.

Stereodon bambergeri: collection from Prm [26] belongs to Hypnum cupressiforme.

Stereodon callichrous: record of this species from Prm $[26,133]$ likely belongs to $S$. callichrous var. japonicus Ando.

Stereodon hamulosum: all records from the Russian Far East need in revision; it is likely that this species does not occur in the area.

Syntrichia laevipila: records from the Russian Far East likely belong to $S$. pagorum.

Thuidium delicatulum: records from Prm [26, 29, 42] were considered by Cherdantseva (unpublished) to be erroneous.

Tortula ucrainica: this species is almost inseparable 
from $T$. cernua and its taxonomic independence requires confirmation [89].

Ulota crispula: material from the Russian Far East likely belongs to undescribed species closely related to U. crispula $(62,99)$.

Ulota reptans was reported from Prm by Gorobets [72], but specimens were not available and record seems doubtful.

Weissia planifolia was reported from Prm by [26] under the name $W$. platyphylla Broth., which is illegitimate.

\section{List of excluded species} (not included in the check-list)

Anacamptodon splachnoides (Froel. ex Brid.) Brid. was recorded for Prm [26] as a doubtful species; Czernyadjeva [53] excluded it from Asian Russia.

Anoectangium stracheyanum Mitt. and $A$. thomsonii Mitt. is a problematic pair of species. Ignatova [100] thought the former name is preferable, however later it became clear that the usage of $A$. thomsonii for Russian plants should be reinstalled.

Anomobryum julaceum (Schrad ex G. Gaertn., B. Mey. \& Scherb.) Schimp.: this name was for a long time used for Russian plants instead of Anomobryum concinnatum, moreover the latter species was occasionally treated as a variety of A. julaceum); A. julaceum s. str. was never found in Russia [55].

Anomodon abbreviatus Mitt.: record from Prm [44] based on erroneous identification of $A$. solovjovii.

Astomum multicapsulare (Sm.) Bruch, Schimp. \& W. Gümbel was reporeted from Prm [147]; however Bardunov \& Cherdantseva considered this record doubtful [26].

Atractilocarpus alpinus (Schimp. ex Milde) Lindb. was excluded from Russian moss flora [90].

Aulacomnium androgynum (Hedw.) Schwägr. was reported from Sakhalin by Sugawara [153]. We consider this record as erroneous, as the species was never found in Asian Russia and we never saw any correctly identified East Asian collections.

Blindiadelphus campylopodus (Kindb.) Fedosov \& Ignatov (Seligeria campylopoda Kindb.) was reported from Khabarovsk Territory [47] and Sakhalin [24] and Kuriles [23], however all so-named specimens were referred later to B. diversifolus [97].

Blindiadelphus recurvatus (Hedw.) Fedosov \& Ignatov was erroneously recorded for Sah instead of Prm [97].

Brotherella yokohamae (Broth.) Broth.: all records of this species from the Russian Far East belong to Pylaisiadelpha tenuirostris.

Brybnia novae-angliae (Sull. \& Lesq.) Grout: according to Huttunen et al. [79], most records of this species from the Russian Far East belong to Brachythecium novae-angliae subsp. bultenii, while some should be referred to $B$. noesicum.

Bucklandiella heterosticha (Hedw.) Bedn.-Ochyra \& Ochyra (Racomitrium heterostichum (Hedw.) Brid.) was excluded from the flora of the Russian Far East [90].

Brachymenium exiloides Bard. \& Czerd. was synonymized with B. exile [99].

Bryum arcticum (R. Br.) Bruch, Schimp. \& W. Gümbel: Bardunov \& Cherdantseva [26] considered the record of Podperra [147] from Prm as erroneous. Zolotov [164, 99] also does not report this species from the southern Russian Far East.
Clastobryella kusatsuensis (Besch.) Z. Iwats.: all records of this species from the southern Russian Far East belong to Pylaisiadelpha tenuirostris.

Ctenidium capillifolium (Mitt.) Broth. was reported from South Sakhalin by Sugawara [153], though specimens could not be located in herbaria; this species was never subsequently found in that area, as well as in other parts of the Russian Far East.

Ctenidium molluscum (Hedw.) Mitt.: records from Am $[7,8,68]$ are dubious. No vouchers were found in MW and VLA.

Cynodontium fallax Limpr. was excluded from the flora of the Russian Far East [90]: so-named specimens from $\mathbf{K h m}$ [88] belong to C. asperifolius.

Dicranum viride (Sull. \& Lesq.) Lindb. was excluded from the flora of the Russian Far East [101]: so-named specimens belong to $D$. hakekodense and D. pacificum.

Didymodon acutus (Brid.) K.Saito: record from Prm [72] seems dubious; no vouchers were found in VLA.

Encalypta streptocarpa Hedw. was excluded from the flora of the Russian Far East [90].

Entodon rufescens Laz. was synonymized with E. sullivantii [83].

Entodon cladorrhizans (Hedw.) Müll. Hal.: all collections from the Russian Far East previously identified as this species actually belong to E. schleicheri.

Entosthodon mublenbergii (Turner) Fife (Funaria mublenbergii Turner): according to the revision by Crundwell \& Nyholm [49], all re-studied collections from Far East were proved to be E. pulchellus, while E. mublenbergii occurs in Russia only in the Caucasus [63].

Fontinalis dalecarlica Bruch, Schimp. \& W. Gümbel: records from Am $[8,7]$ are based on erroneous identifications.

Fissidens bryoides Hedw., F. curvatus Hornsch., F. marginatulus Meln., and $\boldsymbol{F}$. viridulus (Sw.) Wahlenb. belong to Fissidens bryoides-complex, which remains unrevised in Pacific Asia, thus the data on their distribution and even presence in the region have to be considered as preliminary.

Fissidens minutulus Sull. was reported by Podpera [147] for Russky Island near Vladivostok, but the current approach to this species (American in distribution), makes this record improbable.

Gollania neckerella (Müll. Hal.) Broth.: records for Prm [26] were based on misidentified specimens.

Gollania varians (Mitt.) Broth.: records for Prm [26] were based on misidentified specimens.

Grimmia ovalis (Hedw.) Lindb.: records from Am and Prm [103] belong to G. longirostris.

Hedwigia ciliata (Hedw.) P. Beauv. was excluded from the flora of the Russian Far East [114, 114a]: so-named specimens from Pacific Asia belong to other species of Hedwigia.

Hedwigia stellata Hedenäs: all so-named specimens from Far East belong to H. czernyadjevae [114].

Hypnum imponens Hedw. was reported from Prm [26], but no correctly identified specimens were seen from Asian Russia, thus all its records from Far East seems to be erroneous [90]

Isothecium alopecuroides (Lam. ex Dubois) Isov. - this species does not occur in Asian Russia [90]. A record of Lazarenko [131] repeated in Red Data Book of Primorsky 
Territory likely belongs to superficially similar Isothecium bakkodense or Dolichomitriopsis diversiformis.

Kindbergia praelonga (Hedw.) Ochyra (Eurbynchum praelongum (Hedw.) Bruch, Schimp. \& W. Gümbel): record from Prm [26] is based in misidentification.

Leskea gracilescens Hedw. was reported from Am [32], but recent revision revealed that these specimens belong to L. polycarpa [98].

Lewinskya speciosa (Nees) F. Lara, Garilleti \& Goffinet (Orthotrichum speciosum Nees) does not occur in Asian Russia: all so-names specimens from Russian Far East have to be referred to L. elegans [99].

Lindbergia brachyptera (Mitt.) Kindb. was excluded from the Russian moss flora [108].

Lindbergia dutbiei (Broth.) Broth. was excluded from the Russian moss flora [108]; all records belong to Lindbergia sinensis.

Lindbergia japonica Cardot was excluded from the Russian moss flora [108]; all records belong to Lindbergia sinensis [131].

Lyellia crispa R. Br.: record from Khm [117] belongs to L. aspera [97].

Mnium hornum Hedw.: all records from the Russian Far East, e.g. Prm [131], belong to Mnium orientale.

Orthodontium lignicola (Orthodontopsis bardunovii Ignatov \& B.C. Tan) was reported for Khm [99] by mistake.

Oxyrrhynchium hians (Hedw.) Loeske (Eurbynchium bians (Hedw.) Sande Lac.): according to the unpublished revision of Cherdantseva, all records of Oxyrrbynchium bians from the Russian Far East (Am [7, 8, 68], Khs [40], Prm [26, 42]) belong to other species.

Oxyrrhynchium schleicheri (R. Hedw.) Roell (Eurbynchium schleicheri (R. Hedw.) Milde): most records from Russia, inlcuding all from Far East, e.g. Prm [26] were though to be erroneous [90].

Philonotis caespitosa Jur. was reported from Kur by [23], but according to Koponen et al. [124] all so-named specimens from Russian Far East belong to P. fontana.

Philonotis marchica (Hedw.) Brid.: Koponen et al. [124] do not cite this species from Prm since specimens from this area were not available for examination.

Philonotis turneriana (Schwägr.) Mitt. was reported from Iturup by Bakalin et al. [23], but subsequent molecular phylogenetic studies unexpectedly revealed the identity of this specimen with P. marchica [124].

Plagiothecium neckeroideum Bruch, Schimp. \& W. Gümbel: records of this species from Prm [26] were based on erroneous identification of P. eurbyphyllum [90].

Plagiothecium piliferum (Sw.) Bruch, Schimp. \& W. Gümbel: according to Ukrainskaya [160], numerous records from Russia were based on the misidentifications, mostly of Isopterygiopsis muelleriana. This species does not occur in the Russian Far East.

Plagiothecium sylvaticum (Brid.) Bruch, Schimp. \& W. Gümbel: according to Ignatov \& Afonina [80], records of this species belong to $P$. denticulatum or $P$. cavifolium.

Pogonatum neesii (Müll. Hal.) Dozy was reported from Prm [72], however the specimens are not available for our study; difficulty in separation of this species from P. inflexum makes this record dubious.
Poblia leucostoma (Bosch \& Sande Lac.) M. Fleisch. (P. gracillima (Cardot) Horik. \& Ochi) was recorded for Prm [26]; however, according to Czernyadjeva [51], all records of this species from Primorsky Territory belong to $P$. proligera.

Polytrichum formosum Hedw: all records of this species from the Russian Far East belong to P. densifolium [120].

Pylaisia intricata (Hedw.) Bruch, Schimp. \& W. Gümbel: according to Arikawa [19], all records of this species from Asia belong to P. stereodontoides.

Rhizomnium hattori T.J. Kop. was reported from Khs [109], but during the revision of this and some other collections, putatively representing $\mathrm{R}$. hattori, it turned out that they belong to R. tuomikoskii.

Rhizomnium punctatum (Hedw.) T.J. Kop. was reported from Am [8, 36] and Khs [131, MHA], but according to Koponen \& Afonina [122], this species is absent in Far Eastern region [99].

Rhynchostegium riparioides (Hedw.) Cardot (Platyhypnidium riparioides (Hedw.) Dix.) was excluded from the flora of the Russian Far East [90]: all so-named specimens belong to Rhynchostegium aquaticum [78].

Rhynchostegium confertum (Dicks.) Bruch, Schimp. \& W. Gümbel was reported from Prm [132], however the occurrence of this European speices in Pacific Asia seems to be highly unlikely.

Schistidium apocarpum (Hedw.) Bruch, Schimp. \& W. Gümbel was excluded from the moss flora of the Russian Far East [90, 99]: all so-named specimens belong to other species.

Schistidium brunnescens Limpr. was reported from Prm [26] based on [130]. According to [97], it does not occur in the Russian Far East.

Schistidium confertum (Funck) Bruch, Schimp. \& W. Gümbel: all records of this species from the southern Russian Far East have to be referred to other species [97].

Schistidium strictum (Turner) Loeske ex Mårtensson: all records of this species from the Russian Far East belong to other species, mainly S.papillosum and S. lancifolium [97].

Sciuro-hypnum oedipodium (Mitt.) Ignatov \& Huttunen: records from the southern Russian Far East have to be referred to Sciuro-bypnum curtum (Lindb.) Ignatov [85].

Scouleria aquatica Hook.: records of this species from southern Russian Far East have to be referred to $S$. pulcherrima [110].

Seligeria trifaria (Brid.) Lindb. was recorded from Am $[34,130]$, but all studied specimens, which could resemble $S$. trifaria from Am in fact represent large plants of $S$. tristichoides; according to our experience, $S$. trifaria does not occur in the Russian Far East.

Serpoleskea subtilis (Hedw.) Loeske (Amblystegium subtile (Hedw.) Bruch, Schimp. \& W. Gümbel, Amblystegiella subtilis (Hedw.) Loeske, Platydictya subtilis (Hedw.) H.A. Crum): according to unpublished data of V.Ya. Cherantseva, this species is absent in the Russian Far East; all revised specimens belong to Amblystegium serpens.

Sphagnum magellanicum Brid.: all records of this species from the Russian Far East (and likely whole Asian Russia) belong to Sphagnum divinum [72a]; our revision of additional specimens confirms this conclusion.

Stereodon fertilis (Sendtn.) Lindb. (Hypnum fertile Sendtn.): according to [90], this species does not occur in the Russian 
Far East; its records from Prm [26] likely belong to $S$. fauriei.

Thamnobryum plicatulum (Sande Lac.) Z. Iwats. does not occur in Russia [102].

Trichostomum brachydontium Bruch was reported for the Russian Far East by Podpěra [143]; Bardunov \& Cherdantseva [26] considered it as doubtful. The species is known in Russia only from the Caucasus. Later Podperra also did not mention East Asia in the species distribution [144].

Zygodon viridissimus (Dicks.) Brid.: all records of this species from the Russian Far East belong to Z. sibiricus [99].

\section{List of synonyms}

(synonyms are in italic, accepted names are in bold italic)

Amblystegiella jungermannioides = Platydictya jungermannioides Amblystegium saxatile $=$ Amblystegium radicale

Amblystegium juratzkanum = Amblystegium serpens var. juratzkanum

Amblystegium minutissimum = Platydictya minutissima

Amblystegium varium $=$ Hygroamblystegium varium

Anisothecium palustre $=$ Diobelonella palustris

Anisothecium rigidulum = Dicranella humilis

Anisothecium rufescens $=$ Dicranella rufescens

Anisothecium schreberianum = Dicranella schreberiana

Anisothecium varium $=$ Dicranella varia

Anomobryum filiforme $=$ Anomobryum concinnatum

Atrichum hausskenechtii $=$ Atrichum flavisetum

Aulacomnium heterostichum $=$ Arrhenopterum beterostichum

Barbula amplexifolia $=$ Hydrogonium amplexifolium

Barbula convoluta $=$ Streblotrichum convolutum

Barbula cordata $=$ Didymodon cordatus

Barbula cylindrica $=$ Didymodon insulanus

Barbula indica var. kurilensis = Hydrogonium consanguineum var. kurilensis

Barbula reflexa $=$ Didymodon ferrugineus

Barbula rigidula $=$ Didymodon rigidulus

Barbula spadicea $=$ Didymodon spadiceus

Barbula vinealis $=$ Didymodon vinealis

Barbula rigidula var. valida = Didymodon validus

Brachymenium exiloides = Brachymenium exile

Brachythecium novae-angliae subsp. bultenii = Brachythecium bultenii

Brachythecium plumosum $=$ Sciuro-hypnum plumosum

Brachythecium populeum $=$ Sciuro-hypnum populeum

Brachythecium reflexum $=$ Sciuro-hypnum reflexum

Brachythecium starkei $=$ Sciuro-hypnum starkei

Brachythecium thedenii = Brachythecium erythrorrhizon

Brybnia bracbycladula $=$ Myuroclada longiramea

Bryhnia bultenii $=$ Brachythecium bultenii

Brybnia noesica $=$ Brachythecium noesicum

Bryoxiphium savatieri $=$ Bryoxiphium japonicum

Bryum angustirete $=$ Bryum algovicum

Bryum bicolor auct. Fl. Extrmior. = Bryum dichotomum

Bryum inclinatum $=$ Bryum amblyodon

Bryum psendocrispulum = Bryum pseudotriquetrum

Bryum erythrocarpum auct. Fl. Extrmior. = Bryum subapiculatum

Calliergon sarmentosum $=$ Warnstorfia sarmentosa

Calliergon stramineum = Straminergon stramineum

Calliergon trifarium $=$ Pseudocalliergon trifarium

Camptothecium auriculatum $=$ Brachythecium auriculatum

Campylidium hispidulum $=$ Campylophyllopsis hispidulum

Campylidium sommerfeltii $=$ Campylophyllopsis sommerfeltii

Campylium chrysophyllum = Campyliadelphus chrysophyllus

Campylium elodes $=$ Campyliadelphus elodes

Campylium hispidulum = Campylophyllopsis hispidulum

Campylium krylovii = Podperaea krylovii

Campylium polygamum = Drepanocladus polygamus

Campylium radicale $=$ Amblystegium radicale

Campylium sommerfeltii = Campylophyllopsis sommerfeltii
Codriophorus brevisetus $=$ Dilutineuron brevisetum Codriophorus corrugatus $=$ Dilutineuron corrugatum Cratoneuron commutatum = Palustriella commutata Cyrto-bypnum versicolor $=$ Pelekium versicolor Desmatodon ucrainicus $=$ Tortula ucrainica Dichodontium palustre $=$ Diobelonella palustris Dicranella palustris = Diobelonella palustris

Dicranoweisia crispula $=$ Hymenoloma crispulum

Dicranum congestum $=$ Dicranum flexicaule

Ditrichum cylindricum $=$ Trichodon cylindricus

Ditrichum flexicaule = Flexitrichum flexicaule

Ditrichum gracile $=$ Flexitrichum gracile

Drepanocladus badius = Loeskypnum badium

Drepanocladus exannulatus $=$ Warnstorfia exannulata

Drepanocladus fluitans $=$ Warnstorfia fluitans

Drepanocladus intermedius $=$ Scorpidium cossonii

Drepanocladus procerus $=$ Warnstorfia procera

Drepanocladus revolvens $=$ Scorpidium revolvens

Drepanocladus uncinatus = Sanionia uncinata

Drepanocladus vernicosus $=$ Hamatocaulis vernicosus

Entodon compressus = Entodon challengeri

Entodon rufescens $=$ Entodon sullivantii

Entodon sinense $=$ Entodon giraldii

Entodon rubicundus $=$ Entodon flavescens

Eurbynchium eustegium = Eurhynchiadelphus eustegia

Eurbynchium pulchellum = Eurhynchiastrum pulchellum

Fissidens adelphinus $=$ Fissidens teysmannianus

Fissidens cristatus $=$ Fissidens dubius

Forsstroemia mandschurica $=$ Forsstroemia cryphaeoides

Funaria pulchella = Entosthodon pulchellus

Glossadelphus ogatae $=$ Filibryum ogatae

Glyphomitrium warburgii auct. Fl. Extrmior. = Glyphomitrium bumillimum

Gollania amurensis = Gollania ruginosa

Grimmia affinis = Grimmia longirostris

Grimmia sessitana $=$ Grimmia reflexidens

Habrodon leucotrichus = Iwatsukiella leucotricha

Haplocladium auriei = Haplocladium strictulum

Helodium sachalinense = Echinophyllum sachalinense

Heterophyllium haldanianum = Callicladium haldanianum

Heterophyllium nemorosum $=$ Heterophyllium affine

Homalia japonica $=$ Homalia trichomanoides

Homaliadelphus laevidentatus $=$ Homaliadelphus targionianus

Hondaella brachytheciella $=$ Hondaella caperata

Hygrobypnum cocbleariifolium = Platybypnum cochleariifolium

Hygrobypnum dilatatum = Platybypnum duriusculum

Hygrohypnum duriusculum = Platyhypnum duriusculum

Hygrohypnum engyrium = Pseudohygrobypnum eugyrium

Hygrobypnum norvegicum = Platybypnum norvegicum

Hygrohypnum ochraceum = Hygrohypnella ochracea

Hygrohypnum polare = Hygrohypnella polaris

Hygrobypnum subengyrium = Pseudohygrohypnum subeugyrium

Hylocomium pyrenaicum $=$ Hylocomiastrum pyrenaicum

Hypnum demsirameum $=$ Stereodon densirameus

Hypnum fauriei $=$ Stereodon fauriei

Hypnum pallescens $=$ Stereodon pallescens

Hypnum plicatulum $=$ Stereodon plicatulus

Hypnum plumiforme $=$ Stereodon plumiformis

Hypnum pratense $=$ Breidleria pratensis

Hypnum recurvatum $=$ Drepanium recurvatum

Hypnum vaucheri $=$ Stereodon vaucheri

Hypopterygium japonicum = Hypopterygium flavolimbatum

Isothecium diversiforme $=$ Dolichomitriopsis diversiformis

Leptodictyum kochii $=$ Hygroamblystegium humile

Leptodictyum mizushimae = Limnohypnum mizushimae

Leskeella nervosa $=$ Pseudoleskeella nervosa

Macromitrium hymenostomum = Macrocoma tenus subsp. sullivantii 
Mamillariella geniculata $=$ Lindbergia geniculata

Meesia trifaria $=$ Meesia triquetra

Mnium acutum = Plagiomnium acutum

Mnium ambiguum = Mnium lycopodioides

Mnium confertidens = Plagiomnium confertidens

Mnium cuspidatum = Plagiomnium cuspidatum

Mnium drummondii = Plagiomnium drummondii

Mnium immarginatum = Trachycystis ussuriensis

Mnium maximowiczii = Plagiomnium maximowiczii

Mnium medium = Plagiomnium medium

Mnium micro-ovale $=$ Plagiomnium maximowiczii

Mnium minutulum $=$ Rhizomnium parvulum

Mnium orthorrbynchum = Mnium thomsonii

Mnium rostratum = Plagiomnium rostratum

Mnium rugicum = Plagiomnium ellipticum

Mnium striatulum = Rhizomnium striatulum

Mnium ussuriense $=$ Trachycystis ussuriensis

Mnium vesicatum = Plagiomnium vesicatum

Neckera pennata var. tenera $=$ Neckera oligocarpa

Neckera goughiana $=$ Forsstroemia goughiana

Neckera yezoana $=$ Forsstroemia yezoana

Ochyraea alpestris = Platyhypnum alpestre

Ochyraea cocbleariifolia = Platyhypnum cochlearifolium

Ochyraea duriuscula = Platybypnum duriusculum

Ochyraea norvegica $=$ Platyhypnum norvegicum

Ochyraea smithii = Platybypnum smithii

Poblia minor = Poblia elongata var. greenii

Orthodicranum flagellare = Dicranum flagellare

Orthodicranum mayrii = Dicranum mayrii

Orthodicranum montanum $=$ Dicranum montanum

Orthotrichum iwatsukii = Lewinskya iwatsukii

Orthotrichum obtusifolium = Nyholmiella obtusifolia

Orthotrichum rupestre = Lewinskya rupestris

Orthotrichum sordidum = Lewinskya sordida

Orthotrichum speciosum auct. Fl. Extrmior.= Lewinskya elegans

Oxystegus cylindricus $=$ Oxystegus tenuirostris

Physcomitrium eurystomum subsp. acuminatum = Physcomitrium acuminatum

Plagiothecium striatellum $=$ Herzogiella striatella

Plagiothecium succulentum auct. Fl. Extrmior. = Plagiothecium cavifolium

Pogonatum capillare = Pogonatum dentatum

Pogonatum laterale = Pogonatum contortum

Polytrichastrum formosum var. densifolium = Polytrichum densifolium

Polytrichum alpestre = Polytrichum strictum

Polytrichum alpinum = Polytrichastrum alpinum

Polytrichum gracile $=$ Polytrichum longisetum

Polytrichum obioense auct. Fl. Extrmior.= Polytrichum pallidisetum

Pottia truncata $=$ Tortula truncata

Racomitrium brevisetum = Dilutineuron brevisetum

Racomitrium canescens $=$ Niphotrichum canescens

Racomitrium ericoides $=$ Niphotrichum ericoides

Racomitrium fasciculare $=$ Dilutineuron fasciculare

Racomitrium japonicum = Niphotrichum japonicum

Racomitrium laetum = Bucklandiella laeta

Racomitrium microcarpum $=$ Bucklandiella microcarpa

Racomitrium nitidulum = Bucklandiella nitidula

Racomitrium panschii = Niphotrichum panschii

Racomitrium sudeticum $=$ Bucklandiella sudetica

Rhabdoweisia kuzenevae $=$ Rhabdoweisia crispata

Rhynchostegium muelleri $=$ Rhynchostegium aquaticum

Sarmentypnum sarmentosum $=$ Warnstorfia sarmentosa

Schistidium alpicola var. alpicola $=$ Schistidium platyphyllum

Schistidium alpicola var. rivulare $=$ Schistidium rivulare

Seligeria diversifolia $=$ Blindiadelphus diversifolius

Seligeria recurvata $=$ Blindiadelphus recurvatus

Sphanum magellanicum = Sphagnum divinum

Tetrodontium brownianum var. ovatum $=$ Tetrodontium ovatum
Thamnobryum obtusatum = Thamnobryum neckeroides

Thamnobryum vorobjovii = Thamnobryum neckeroides

Thamnobryum sandei = Thamnobryum subseriatum

Thuidium bipinnatulum = Pelekium versicolor

Thuidium glaucinum $=$ Thuidium pristocalyx

Thuidium philibertii $=$ Thuidium assimile

Thuidium pygmaeum = Pelekium pygmaeum

Thuidium vestitissimum $=$ Bryochenea vestitissima

Tortula laevipila = Syntrichia laevipila

Tortula ruralis = Syntrichia ruralis

Tortula sinensis = Syntrichia sinensis

\section{ACKNOWLEDGEMENTS}

The study of was supported by RSF 18-14-00121, for OP, MI, EI and VF, by RFBR 17-04-00018 for VB and also is a part of biocollection development (AAAA-A17-117073110003-8, for VBGI, and AAAA-A17-117012610052-2 and USU 440534 for OP).

\section{LITERAT URE CITED}

${ }^{1}$ Abramova, A.L. \& I.I. Abramov 1951 Rare species of the moss flora of Far East. Botanicheskie Materialy Otdela Sporovykh Rastenij Botanicheskogo Instituta Akademii Nauk SSSR 7:219-223 (in Russian). [Абрамова А.А., Абрамов И.И. 1951. Редкие виды мхов дальневосточной флоры // Ботанические материалы Отдела споровых растений Ботанического института АН СССР. Т. 7. С. 219-223].

${ }^{2}$ Abramova, A.L. \& I.I. Abramov 1966. Rare moss species of Siberia and the Far East. Novosti Sistematiki Nizshih rastenii 3:302-315 (in Russian). [Абрамова А.А., Аб̆рамов И.И. 1966. РеАкие виды мхов Сибири и Аальнего Востока // Новости систематики низших растений. T. 3. C. 302-315].

${ }^{3}$ Abramova, A.L. \& I.I. Abramov 1969. A new moss for the Far East - Archidium alternifolium (Hedw.) Mitt. Novosti Sistematiki Nizshih rastenii 6:262-265 (in Russian). [Абрамова А.А., Абрамов И.И. 1969. Новый мох Аля Аальнего Востока - Archidium alternifolium (Hedw.) Mitt. // Новости систематики низших растений. Т. 6. С. 262-265].

${ }^{4}$ Abramova, A.L. \& I.I. Abramov 1977. To the bryoflora of the Far East. Novosti Sistematiki Nizshih rastenii 14:212-219 (in Russian). [Абрамова А.А., Аб̆рамов И.И. 1977. К бриофлоре Аамьнего Востока // Новости систематики низших растений. Т.14. С. 212-219].

${ }^{5}$ Abramova, A.L. \& I.I. Abramov 1978. On the species of the family Thuidiaceae in the USSR. Novosti Sistematiki Nizshib rastenii 15:208-211 (in Russian). [Абрамова A.А., Абрамов И.И. 1978. О видах семейства Тhuidiaceae в СССР // Новости систематики низших растений. T. 15. C. 208-211].

${ }^{6}$ Abramova, A.L. \& I.I. Abramov 1984. A new for USSR species of genus Hypnum. Novosti Sistematiki Nizshib rastenii 21:192-197 (in Russian). [Абрамова А.А., И.И. Абрамов 1984. Новый вид рода Нypnum Hedw. Аля СССР // Новости систематики низших растений. Т. 21. C. 192-197].

${ }^{7}$ Abramova, L.I. \& D.A. Petelin 1981. Bryophytes. In: Flora and vegetation of Tukuringra ridge (Amur Province) (I.A. Gubanov, ed.), Moscow State University, Moscow, pp. 64-85, (in Russian). [Абрамова А.И., Петелин А.А. 1981. Мохообразные // ФАора и растительность хребта Тукурингра (Амурская об̆ласть) / под реА. И.А. Губанова. Москва, ИзАательство МГУ. С. 64-85].

${ }^{8}$ Abramova, L.I., L.V. Bardunov, A.I. Gubanov, M.S. Ignatov, S.N. Korenyuk, V.S. Novikov, D.A. Petelin \& N.N. Ste- 
tsura 1987. Bryophytes and vascular plants of Zeya nature reserve. In: Flora and fauna of USSR nature reserves (I.A. Gubanov \& D.A. Petelin, eds.). Moscow, Vyp.1, 70 pp. (in Russian). [Абрамова А.И., Бардунов А.В., Губанов А.И., Игнатов М.С., Коренюк С.Н., Новиков В.С., Петелин А.А., Стецура Н.Н. 1987. Мохообразные и сосудистые растения Зейского заповедника. ФАора и фауна заповедников СССР / под реА. И.А. Губанова и А.А. Петелина. Москва, вып. 1. 70 с.].

${ }^{9}$ Afonina, O.M. 2004. Hypnum sect. Hamulosa (Musci, Hypnaceae) in Russia. Arctoa 13:9-28 (in Russian with English summary). [Афонина O.M. 2004. Вилы Нурпит секции Hamulosa (Musci, Hypnaceae) в России // Arctoa. Vol. 13. C. 9-28].

${ }^{10}$ Afonina, O.M. 2008 Stereodon (Brid.) Mitt. In: Moss flora of Russia (in Russian). [Афонина O.M. 2008. Род Stereodon (Brid.) Mitt. - Стереодон. ФАора мхов России. 29 с.] http:/ /arctoa.ru/Flora/taxonomy-ru/Stereodon-textrussian.pdf

${ }^{11}$ Afonina, O.M. 2009. New moss records from Khabarovsk Territory. 2. Arctoa 18:279.

${ }^{12}$ Afonina, O.M. \& E.A. Ignatova 2007a. A new species of Didymodon (Pottiaceae, Musci) from Asian Russia. Arctoa 16:133-138.

${ }^{13}$ Afonina, O.M. \& E.A. Ignatova 2007b. East Asian species of Stereodon (Brid.) Mitt. (Pylaisiaceae, Musci) in Russia. Arctoa 16:7-20.

${ }^{14}$ Afonina, O.M. \& E.A. Ignatova 2009. Syntrichia amphidiacea (Pottiaceae), a new species for the moss flora of Russia. Botanicheskii Zhurnal 94(3):439-443 (in Russian with English summary). [Афонина O.M., Игнатова E.A. 2009. Syntrichia amphidiacea (Pottiaceae) - новый вил Аля фморы мхов России // Ботанический журнал Т. 94, № 3. 439-443].

${ }^{15}$ Afonina, O.M., H. Tsubota \& E.A. Ignatova 2007. The genus Pylaisiadelpha (Pylaisiadelphaceae, Musci) in Russia. Arctoa 16:127-132.

${ }^{16}$ Afonina, O.M., V.Ya. Cherdantseva, E.A. Ignatova \& M.S. Ignatov 2010. Symblepharis vaginata (Dicranaceae, Bryophyta), a new genus and species for the moss flora of Russia. Botanicheskii Zhurnal 95(12):1765-1770 (in Russian with English summary). [Афонина O.M., Черданцева В.Я., Игнатова Е.А., Игнатов М.С. 2010. Symblepharis vaginata (Dicranaceae, Bryophyta) - новый род и виА Аля фморы мхов России // Ботанический журнац. Т. 95, № 12. С. 1765-1770].

${ }^{17}$ Afonina, O.M., I.V. Czernyadjeva, E.A. Ignatova \& J. Kučera 2010. Five species of Didymodon (Pottiaceae, Bryophyta) new to Russia. Arctoa 19:51-62.

${ }^{18}$ Afonina, O.M., E.A. Ignatova, V.E. Fedosov \& O.I. Kuznetsova. 2014. Toward a new understanding of Syntrichia submontana (Pottiaceae, Bryophyta). Arctoa 23:11-24.

${ }^{19}$ Arikawa, T. 2004. A taxonomic study of the genus Pylaisia (Hypnaceae, Musci). Journal of the Hattori Botanical Laboratory 95:71-154.

${ }^{20}$ Bakalin, V.A. 2013. Valentina Yakovlevna Cherdantseva (1939-2013), in memoriam. Arctoa 22:229-238.

${ }^{21}$ Bakalin, V.A. \& V.Ya. Cherdantseva 2006. Bryophytes of northern Kuril Islands (north-west Pacific). Arctoa 15:131-153.

${ }^{22}$ Bakalin, V. A. \& T. Katagiri 2014. The historical collection of liverworts from Sakhalin by Urbian Faurie (18461915) in herbarium of Kyoto. Arctoa 23:29-32.
${ }^{23}$ Bakalin, V.A., V.Ya. Cherdantseva, M.S. Ignatov, E.A. Ignatova \& T.I. Nyushko 2009. Bryophyte flora of the South Kuril Islands (East Asia). Arctoa 18:69-114.

${ }^{24}$ Bakalin, V.A., O.Yu. Pisarenko, V.Ya. Cherdantseva, P.V. Krestov, M.S. Ignatov \& E.A. Ignatova 2012. Bryophytes of Sakhalin. Izd-vo Morskogo gosuniversiteta, Vladivostok, 310 pp. (in Russian). [Бакалин В.А., Писаренко О.Ю., Черданцева В.Я., Крестов П.В., Игнатов М.С., Игнатова Е.А. 2012. Бриофлора Сахалина. Владивосток: ИзА-во Морского госуниверситета, 2012. 310 с.].

${ }^{25}$ Bakalin, V., V. Fedosov, O. Pisarenko \& E. Borovichev 2018. The bryophyte flora of the northern Iturup (North-West Pacific): somewhere between Circumboreal and East Asian Floristic Regions. Journal of Bryology, in press.

${ }^{26}$ Bardunov, L.V. \& V.Ya.Cherdantseva 1982. Mosses of the South of Primorskij Province. Novosibirsk, Nauka. 208 pp. (in Russian) [Бардунов А.В., Черданцева В.Я. 1982. Аистостебельные мхи Южного Приморья. Новосибирск, Наука. 208 с.].

${ }^{27}$ Bardunov, L.V. \& V.Ya.Cherdantseva 1984. Materials on the moss flora of the South Kuril Islands. In: Sistematic-floristic studies of spore plants of the Far East (V.Ya. Cherdantseva, ed.), Vladivostok, pp. 34-53 (in Russian). ББардунов А.В., Черданцева В.Я. 1984. Материалы по фморе Аистостебельных мхов Южных Курильских островов // Систематико-флористические исследования споровых растений АаАьнего Востока / под реА. В.Я. Черданцевой. ВАадивосток. С. 34-53].

${ }^{28}$ Bardunov, L.V. \& V.Ya. Cherdantseva 2006. Bryophytes. In: Flora, rastitel'nost' $i$ micobiota zapovednika 'Ussuryjsky". (L.N. Vasilyev, ed.), Dal'nauka, Vladivostok, pp. 51-78 (in Russian). [Бардунов А.В., Черданцева В.Я. 2006. Мохообразные // ФАора, растительность и микобиота заповедника "Уссурийский" / под ред. А.Н. Васильевой. ВАадивосток: Аальнаука. С. 51-78].

${ }^{29}$ Bardunov, L.V., S.K. Gambaryan \& V.Ya.Cherdantseva 1991. Bryophytes. In: Flora, miko- and likhenobiota of Lazo nature reserve (Primorskij Kraj) (Z.M. Azbukina, ed.). Vladivostok AN SSSR, DVO, Biologo-Pochvennyj Institut \& Lazovskij Gosudarstvennyj Zapovednik, pp. 35-60 (in Russian). [Бардунов А.В., Гамбарян С.К., Черданцева В.Я. 1991. Мохообразные // ФАора, мико- и михенобиота Аазовского заповедника (Приморский край) / под реА. 3. М. Азбукиной. ВАаАивосток, АН СССР, $\triangle \mathrm{BO}$, Биолого-почвенный институт и Аазовский государствененый заповедник. С. 35-60].

${ }^{30}$ Bardunov, L.V., V.Ya. Cherdantseva \& S.G. Kazanovsky 2009. Pseudoleskeopsis rippelii (Leskeaceae, Musci), the first reliable record in Russia. Botanicheskii Zhurnal 94(1):101106 (in Russian with English summary). [Бардунов А.B., Черданцева В.Я., Казановский С.Г. 2009. Первое Аостоверное указание Psendoleskeopsis zippelii (Leskeaceae, Musci) Аля России // Ботанический журнал. Т. 94, № 1. С. 101-106].

${ }^{31}$ Bezgodov, A.G. \& E.A. Ignatova 2013. Poblia lutescens (Mielichhoferiaceae, Bryophyta) in Russia. Arctoa 22:107-110.

${ }^{32}$ Bezgodov, A.G., E.A. Ignatova \& M.S. Ignatov 2013. Mosses of the Nora Nature Reserve. In: Sbornik statei k 15-letivu Norskogo Zapovednika (N.N. Kolobaev, ed.), Blagoveshchensk \& Fevralsk, Zeya, pp. 59-78 (in Russian). Безгодов А.Г., Игнатова Е.А., Игнатов М.С. 2013. Список мхов Норского заповедника // Сборник статей к 15-летию Норского заповедника /под ред. Н.Н. Комобаева. Благовещенск-Февральск: Зея. С. 59-78]. 
${ }^{33}$ Blom, H.H., E.A. Ignatova \& O.M. Afonina 2006. New records of Schistidium (Grimmiaceae, Musci) in Russia. Arctoa 15:187-194.

${ }^{34}$ Brotherus, V.F., O.I. Kuzeneva \& N.I. Prokhorov 1916. List of mosses from Amur and Yakutsk Provinces. Trudy Botanicheskogo muzeya Akademii nauk 16:1-71 (in Russian). [Бротерус В.Ф., Кузенева О.И., Прохоров Н.И. 1916. Список мхов из Амурской и Якутской областей // Труды Ботанического музея Академии наук. Вып. 16. C. 1-71].

${ }^{35}$ Cherdantseva, V.Ya. 1973. On the bryoflora of the forests of Verkhne-Zejskaya plain. Voprosy Geografii Dal'nego Vostoka 1:15-19 (in Russian). [Черданцева В.Я. 1973. К бриофлоре лесов Верхне-Зейской равнины // Вопросы географии Аахьнего Востока. Вып. 1. С. 15-19].

${ }^{36}$ Cherdantseva, V.Ya. 1976. Checklist of leafy mosses of South Sakhalin. Nizshiye Rastenuya Dalnego Vostoka, Vladivostok, 140-177 (in Russian). [Черданцева В.Я. 1976. Конспект флоры мистостебельных мхов южного Сахалина // Низшие растения Аальнего Востока. ВАадивосток. С. 140-177].

${ }^{37}$ Cherdantseva, V.Ya. 1989. Rare and interesting moss species in the Far East. Novosti Sistematiki Nizshib rastenii 26:157-159 (in Russian with English summary). [ЧepАанцева В.Я. 1989. Редкие и интересные виды мхов Аальнего Востока // Новости систематики низших растений. Т. 26. С. 157-159].

${ }^{38}$ Cherdantseva, V.Ya. 2002. Mosses of Sikhote-Alin State Biopshere Reserve. Arctoa 11:229-244 (in Russian with English summary). [Черданцева В.Я. 2002. Аистостебельные мхи Сихотэ-Алинского биосферного заповедника (Аальний Восток, Приморский край) // Arctoa Vol. 11. С. 229-244].

${ }^{39}$ Cherdantseva, V.Ya. 2007. Mosses. In: Flora, mikobiota and vegetation of Bastak nature reserve (T.A. Rubtsova, ed.), Dal'nauka, Vladivostok, pp. 83-100 (in Russian). [ЧepАанцева В.Я. 2007. Аистостебельные мхи // Фмора, микобиота и растительность заповедника Бастак / под реА. Т.А. Рубцовой. ВАадивосток: Аальнаука. C. 83-100].

${ }^{40}$ Cherdantseva, V.Ya. \& S.K. Gambaryan 1986. Bryophytes. In: Flora and vegetation of Bolshoi Khekhtsir Nature Reserve (A.B. Melnikov, ed.), Vladivostok, pp. 79-101 (in Russian). [Черданцева В.Я., Гамбарян С.К. 1986. Мохообразные // ФАора и растительность Большехехцирского заповедника / под реА. А.Б. Мельникова. ВАадивосток. C. 79-101].

${ }^{41}$ Cherdantseva, V.Ya. \& S.K. Gambaryan 1989. Bryophytes. In: Fungi, lichens, algae and bryophytes of Komsomolsk reserve (Khabarousk Territory) (Z.M. Azbukina, V.Ya.Cherdantseva, eds.), Vladivostok, pp. 109-135 (in Russian). [Черданцева В.Я., Гамбарян С.К. 1989. Мохообразные // Грибы, мишайники, водоросли и мохообразные Комсомольского заповедника (Хабаровский край) / поА реА. 3.М. Азбукиной и В.Я. Черданцевой. ВАадивосток. C. 109-135].

${ }^{42}$ Cherdantseva, V.Ya., L.V.Bardunov \& S.K. Gambaryan 1984. Mosses. In: Flora Verkhneussurijskogo statsionara (Yuzhnij Sikhote-Alin'), Dal'nevostochnyi Nauchnyi Tsentr Akademii Nauk SSSR, pp. 75-95 (in Russian). [Черданцева В.Я., Бардунов А.В., Гамбарян С.К. 1984. Мхи // ФАора Верхнеуссурийского стационара (Южный СихотэА^инь), В^адивосток, АВНЦ АН СССР. С. 75-95].

${ }^{43}$ Cherdantseva, V.Ya., S.K. Gambaryan \& S.V. Osipov 1997. The bryophytes of the upper belts of the Amur-
Uda interfluve Mountains (Far East). Botanicheskii Zhurnal 82(1):54-62. (in Russian with English summary). [ЧepАанцева В. Я., Гамбарян С. К., Осипов С.В. 1997. Мохообразные верхних поясов гор Амуро-УАского межАуречья (Аальний Восток) // Ботанический журнал. T. 82, № 1. C. 54-62].

${ }^{44}$ Cherdantseva, V.Ya., K.V. Gorobets, J. Harpel, M.S. Ignatov, E.A. Ignatova \& V.V. Teleganova 2006a. New moss records from Sakhalinskaya Province. 1. Sakhalin. Arctoa 15:263-268.

${ }^{45}$ Cherdantseva, V.Ya., M.S. Ignatov \& E.A. Ignatova 2006b. New moss records from Primorsky Territory. 1. Arctoa 15:263.

${ }^{46}$ Cherdantseva, V.Ya., M.S. Ignatov \& E.A. Ignatova 2006c. New moss records from Sakhalin Province. 2. Kunashir. Arctoa 15:268.

${ }^{47}$ Cherdantseva, V.Ya, M.S. Ignatov \& V.A. Bakalin 2009. New moss records from Khabarovsk Territory. 1. Arctod 18:279.

${ }^{48}$ Cherdantseva, V.Ya, V.A. Bakalin \& L.S. Yakovchenko 2013. Bryophyte flora and lichen biota of Litovka Mt. (Livadiysky Range, Russian Manchuria). Komarovskie Chteniya 61:9-49 (in Russian with English summary). [Черданцева В.Я., Бакалин В.А., Яковченко А.С. 2013. Бриофлора и михенобиота горы Аитовка (АиваАийский хребет, российская Манчжурия) // Комаровские чтения T. 61. C. 9-49].

${ }^{49}$ Crundwell, A.C. \& E. Nyholm 1974. Funaria mublenbergii and related European species. Lindbergia 2:222-229.

${ }^{50}$ Czernyadjeva, I.V. 1997. The species of the genus Poblia (Musci) with propagules. Botanicheskii Zhurnal 82(1):102122 (in Russian with English summary). [Чернядьева И.В. 1997. Вилы рода Poblia (Musci) с выводковыми почками. Ботанический журнал. Т. 82, №1. С. 102122].

${ }^{51}$ Czernyadjeva, I.V. 1999. On the distribution of propaguliferous species of Poblia (Bryaceae, Musci) in Russia. Arctoa 8:51-56.

${ }^{52}$ Czernyadjeva, I.V. 2004. The genus Hygrohypnum (Amblystegiaceae, Musci) in Russia. Arctoa 12:25-58 (in Russian with English summary). [Чернядьева И.В. 2004. Рол Hygrohypnum (Amblystegiaceae, Musci) в России // Arctoa. Vol. 12. C. 25-58].

${ }^{53}$ Czernyadjeva, I.V. 2007. The genus Anacamptodon (Amblystegiaceae, Bryophyta) in Russia and Transcaucasia. Arctoa 16:1-6.

${ }^{54}$ Czernyadjeva, I.V. \& E.A. Ignatova 2013. Dichelyma (Fontinalaceae, Bryophyta) in Russia. Arctoa 22:111-120.

${ }^{55}$ Czernyadjeva, I.V., O.M. Afonina \& E.A. Ignatova 2015. The genus Anomobryum (Bryaceae, Musci) in Russia. Arctoa 24:459-470.

${ }^{56}$ Dudov, S.V., M.N. Kozhin, V.E. Fedosov \& E.A. Ignatova 2018. Moss flora of Zeysky state nature reserve (Tukuringra Range, Amur Province, Russia). Botanica Pacifica 7(2): 1-22.

${ }^{57}$ Dudov, S.V., E.A. Ignatova \& V.E. Fedosov 2015. New moss records in Amur Province. 2. Arctoa 24:251-252 (in Russian). [Аудов С.В., Игнатова Е.А., Федосов В.Э. 2015. Новые находки мхов в Амурской области. 2. In: New bryophyte records. 4 (E.V. Sofronova, ed.) // Arctoa Vol. 24. P. 251-252].

${ }^{58}$ Dudov S.V., V.E. Fedosov \& M.N. Kozhin 2016. New 
moss records from Amur province. 3. In: New bryophyte records. 7 (E.V. Sofronova, ed.). Arctoa 25:446.

${ }^{59}$ Ellis, L.T., M. Aleffi, H. Bednarek-Ochyra, V.A. Bakalin, M. Boiko, J.A. Calleja, V.E. Fedosov, M.S. Ignatov, E.A. Ignatova, R. Garilleti, T. Hallingbäck, N. Lönnell, N. Hodgetts, T. Kiebacher, J. Larraín, M. Lebouvier, M. Lüth, V. Mazimpaka, B. Vigalondo, F. Lara, R. Natcheva, M. Nobis, A. Nowak, J.D. Orgaz, J. Guerra, J. Pantović, N. Nikolić, M.S. Sabovljević, A.D. Sabovljević, O.Yu. Pisarenko, V. Plášek, Z. Skoupá, S. Poponessi, M. Privitera, M. Puglisi, M. Skudnik \& Q.H. Wang 2017. New national and regional bryophyte records, 51. Journal of Bryology 39(2):177-190

${ }^{60}$ Fedosov, V.E. 2012. Encalypta sect. Rhabdotheca in Russia. Arctoa 21:101-112.

${ }^{61}$ Fedosov, V.E. \& E.A. Ignatova 2008. The genus Bryoerythrophyllum (Pottiaceae, Bryophyta) in Russia. Arctoa 17:19-38.

${ }^{62}$ Fedosov, V.E. \& E.A. Ignatova. 2018. On the genus Ulota (Orthotrichaceae, Bryophyta) in Russia. Novosti Sistematiki Niqshikh Rastenij 52(1):141-171.

${ }^{63} \mathrm{Fedosov}$, V.E., E.A. Ignatova, M.S. Ignatov \& G.Ya. Doroshina. 2010. On the genus Entosthodon (Funariaceae, Musci) in the Caucasus. Arctoa 19:75-86.

${ }^{64}$ Fedosov, V.E., I.V. Czernyadjeva, E.A. Ignatova, O.I. Kuznetsova, A.V Fedorova \& M.S. Ignatov 2016. On the Bryoxiphium norvegicum and B. japonicum (Bryoxiphiaceae, Bryopsida). Arctoa 25(1):52-68.

${ }^{65}$ Fedosov V.E., A.V. Ermolenko, D.E. Koltysheva \& V.A. Bakalin 2016. On the moss flora of Tardoki-Yani Mountain (North Sikhote-Alin, Russian Far East). Arctoa 25(2):420-428.

${ }^{66} \mathrm{Fedosov}$ V.E., O.Yu. Pisarenko, V.A. Bakalin \& E.A. Ignatova 2016. Venturiella sinensis (Erpodiaceae, Bryophyta), a new species and a new family for the moss flora of Russia. Arctoa 25:334-368

${ }^{66}$ Fedosov, V.E., G.Ya. Doroshina, D.Ya. Tubanova, O.M. Afonina \& E.A. Ignatova. 2017. Four Orthotrichaceae species new for Russian flora. Arctoa 26(2): 154-165.

${ }^{67}$ Fedosov, V.E., A.V. Fedorova., E.A. Ignatova \& M.S. Ignatov 2017. A revision of the genus Seligeria (Seligeriaceae, Bryophyta) in Russia inferred from molecular data. Phytotaxa 323(1):27-50.

${ }^{68}$ Gambaryan, S.K. 1978. On the bryoflora of Zeyskiy nature reserve (Amur Province). In: Vodorosli, griby i mbi Dalnego Vostoka. pp. 109-112, DVO AN SSSR, Vladivostok (in Russian). [Гамбарян С.К. 1978. О бриофлоре Зейского заповедника (Амурская область). В кн.: Водоросли, грибы и мхи Аальнего Востока. ВАаАивосток, $\triangle \mathrm{BO}$ АН CССР. C. 109-112.]

${ }^{69}$ Gambaryan, S.K. 1981. Mosses of bog phytocenoses of the middle course Zeya River. In: Sistematika, ekologiya $i$ geografiva sporovykh rastenij Dal'nego Vostoka (ed. V.Ya Cherdantseva). Dal'nevostpchnyj Nauchnyj Tsentr Akademii Nauk SSSR, Vladivostok. pp. 78-81 (in Russian). Гамбарян С.К. 1981. Аистостебельные мхи бомотных фитоценозов Аолины среднего течения реки Зея // Систематика, экология и география споровых растений Аальнего Востока (под реА. В.Я. Черданцевой). ВАаАивосток: АВНЦ АН СССР. С. 78-81].

${ }^{70}$ Gambaryan, S.K. \& V.Ya. Cherdantseva 1998. Bryophytes. In: Flora and vegetation of Khingan nature reserve (L.N. Vasilieva, ed.), pp. 70-87, Dal'nauka, Vladivostok (in Russian).
[Гамбарян С. К., Черданцева В. Я. 1998. Мохообразные / / ФАора и растительность Хинганского заповедника (Амурская область) / пол реА. А.Н. Васильевой. ВАаАивосток: Аальнаука. С. 70-87].

${ }^{71}$ Gambaryan, S.K. \& V.Ya. Cherdantseva 2002. Bryophytes. In: The inventory of plants and fungi of the "Kedrovaya pad" nature reserve (R.I. Korkishko, ed.). Vladivostok: Dal'nauka, pp. 21-30 (in Russian). ГГамб̆арян С.К., Черданцева В.Я. 2002. Мохообразные / / КаАастр растений и грибов заповедника Кедровая падь / под ред. Р.И. Коркишко. ВАадивосток: Аальнаука. С.21-30].

${ }^{72}$ Gorobets, K.V. 2004 Moss flora of Murajov-Amursky's Peninsula and islands of Petra Velikogo Bay (Primorsky Territory). Ph.D. Thesis, Vladivostok, Tikhookeansky Institut Bioorganicheskoj Khimii Dalnevostochnogo Otdeleniya Akademii Nauk. 22 pp. (in Russian). ГГоробец K.B. 2004. ФАора Аистостебельных мхов п-ова МуравьеваАмурского и островов залива Петра Великого (Приморский край). Автореферат Аиссертации кандидата биологических наук. ВАадивосток: ТИБОХ $\triangle \mathrm{BO}$ PAH. 22 c.].

${ }^{72 a}$ Hassel, K., M.O. Kyrkjeeide, N. Yousefi, T. Prestø, H.K. Stenøien, J. Shaw \& K.I. Flatberg 2018. Sphagnum divinum (sp. nov.) and $S$. medium Limpr. and their relationship to $S$. magellanicum Brid. Journal of Bryology 40(3): 197-222.

${ }^{73}$ Hedenäs, L. 1997. A partial generic revision of Campylium (Musci). Bryologist 100:65-88.

${ }^{74}$ Hedenäs, L. 2017. Scandinavian Oncophorus (Bryopsida, Oncophoraceae): species, cryptic species, and intraspecific variation. European Journal of Taxonomy 315:1-34.

${ }^{75}$ Higuchi, M. \& V. A. Bakalin 2013. Taxonomic position and a new locality of Gollania taxiphylloides Ando \& Higuchi (Hypnaceae, Bryophyta). Hikobia 16(3): 289-291.

${ }^{75 a}$ Higuchi, M. \& V.A. Bakalin 2017. Mosses of Kedrovaya Pad nature reserve, Primorskiy Territory, Far East Russia. Bulletin of the National Museum of Nature and Science, Series B (Botany) 43(1):11-18.

${ }^{76}$ Higuchi, M. \& K. Sato. 2004. A small collection of mosses from Kunashir Island, the Kuriles. Bulletin of the National Science Museum, Tokyo. Series B, Botany 30:103-107.

${ }^{77}$ Horikawa, Y. 1934. Symbolae florae Bryophytae OrientaliAsiae. The Botanical Magarine, Tokyo. I. 48(571): 452-462. II 48(573): 599-609. III 48(574): 708-719. IV 49(585): $588-595$.

${ }^{78}$ Huttunen, S. \& M.S. Ignatov 2010. Evolution and taxonomy of aquatic species in the genus Rhynchostegium (Brachytheciaceae, Bryophyta). Taxon 59(3):791-808.

${ }^{79}$ Huttunen, S., O. Kuznetsova, M. Li, Y-F. Wang \& M. Ignatov 2015. East Asian origin and Northern Hemisphere diversification in the Brachythecium novae-angliae-complex (Bryophyta). Taxon 64(5):911-930.

${ }^{80} \mathrm{Ignatov}$, M.S. \& O.M. Afonina (eds.). 1992. Check-list of mosses of the former USSR. Arctoa 1:1-85.

${ }^{81}$ Ignatov, M.S. \& V.Ya. Cherdantseva 1995. The families Cryphaeaceae, Leucodontaceae and Leptodontaceae (Musci) in Russia. Arctoa 4:65-104.

${ }^{82}$ Ignatov, M.S. \& E.A. Ignatova 2009. Two new taxa of Pottiaceae from the Kuril Islands. Arctoa 18:135-140.

${ }^{83}$ Ignatov, M.S. \& E.A. Ignatova 2018. On the identity of Entodon rufescens Laz. Arctoa 27:87-90.

${ }^{84}$ Ignatov, M.S. \& I.A. Milyutina 2007 a. A revision of the genus Sciuro-hypnum (Brachytheciaceae, Bryophyta) in Rus- 
sia. Arctoa 16:63-86.

${ }^{85}$ Ignatov, M. S. \& I.A. Milyutina 2007b. On Sciuro-hypnum oedipodium and $S$. curtum (Brachytheciaceae, Bryophyta). Arctoa 16:47-61.

${ }^{86}$ Ignatov, M.S. \& A.I. Milutina 2010. The genus Brachythecium (Brachytheciaceae, Musci) in Russia: comments on species and key for identification. Arctoa 19:1-30.

${ }^{87}$ Ignatov, M.S. \& T. Suzuki 2018. Fissidens beckettii (Fissidentaceae, Bryophyta). Novosti sistematiki nizshikh rastenii 52(2): 463-468 (in Russian with English summary). [Игнатов M.C., Сузуки Т. 2018. Fissidens beckettii (Fissidentaceae, Bryophyta) - новый виА Аля флоры мхов России // Новости систематики низших растений Т. 52 , №2. С. 463-468].

${ }^{88}$ Ignatov, M.S., B.C. Tan, Z. Iwatsuki \& E.A. Ignatova 2000. Moss flora of the Upper Bureya River (Russian Far East). Journal of the Hattori Botanical Laboratory 88:147-178.

${ }^{89}$ Ignatov, M.S., E.I. Ivanova, E.A. Ignatova \& K.K. Krivoshapkin 2001. On the moss flora of Ust-Maya District (Republic Sakha-Yakutia, East Siberia). Arctoa 10: 165-184.

${ }^{90}$ Ignatov, M.S., O.M. Afonina, E.A. Ignatova, M.S. Ignatov, O.M. Afonina, E.A. Ignatova, A. Abolina, T.V. Akatova, E.Z. Baisheva, L.V. Bardunov, E.A. Baryakina, O.A. Belkina, A.G. Bezgodov, M.A.Boychuk, V.Ya. Cherdantseva, I.V. Czernyadjeva, G.Ya. Doroshina, A.P. Dyachenko, V.E. Fedosov, I.L. Goldberg, E.I. Ivanova, I. Jukoniene, L. Kannukene, S.G. Kazanovsky, Z.Kh. Kharzinov, L.E. Kurbatova, A.I. Maksimov, U.K. Mamatkulov, V.A. Manakyan, O.M. Maslovsky, M.G. Napreenko, T.N. Otnyukova, L.Ya. Partyka, O.Yu. Pisarenko, N.N. Popova, G.F. Rykovsky, D.Ya. Tubanova, G.V. Zheleznova \& V.I. Zolotov 2006. Check-list of mosses of East Europe and North Asia. Arctoa 15:1-130.

${ }^{91}$ Ignatov, M.S., E.A. Ignatova \& V.Ya. Cherdantseva 2006. Oedipodium griffithianum (Dicks.) Schwägr. (Oedipodiopsida, Bryophyta), a new species and class for Russia. Arctoa 15:211-214.

${ }^{92}$ Ignatov, M.S., T. Suzuki \& V.Ya Cherdantseva 2007. Fissidens byalinus (Fissidentaceae, Bryophyta), a new species for Russia. Arctoa 16:123-126.

${ }^{93}$ Ignatov, M.S., I.A. Milyutina \& V.K. Bobrova 2008. Problematic groups of Brachythecium and Eurbynchiastrum (Brachytheciaceae, Bryophyta) and taxonomic solutions suggested by nrITS sequences. Arctoa 17:113-138.

${ }^{94}$ Ignatov M.S., E.A. Ignatova \& E.V. Malashkina 2013. Ephemerum spinulosum Bruch \& Schimp. (Bryophyta) - a new species for Russia. Arctoa 22: 97-100.

${ }^{95}$ Ignatov M.S., O.I. Kuznetsova \& I.V. Czernyadjeva 2014. On the systematic position of Leptodictyum mizushimae. Arctoa 23: 185-193.

${ }^{96}$ Ignatov, M., S. Huttunen, M. Li \& Y-F. Wang 2015. How to know Myuroclada longiramea (Brachytheciaceae, Bryophyta). Arctoa 24:497-502.

${ }^{97}$ Ignatov, M.S., E.A. Ignatova, V.E. Fedosov, E.I. Ivanova, H.H. Blom, J. Muños, H. Bednarek-Ochyra, O.M. Afonina, L.E. Kurbatova, I.V. Czernyadjeva \& V.Ya. Cherdantseva 2017. Moss flora of Russia. Vol. 2. Oedipodiales - Grimmiales. Moscow, 560 pp. (in Russian and English). [Игнатов М.С., Игнатова Е.А., Федосов В.Э., Иванова Е.И., БАом Х.Х., Муньос И., БеАнарек-Охыра Х., Афонина О.М., Курбатова А.Е., Чернядьева И.В., Черданцева В.Я. 2017. ФАора мхов России. Том 2. Oedipodiales - Grimmiales. Москва: КМК. 560 с.].
${ }^{98}$ Ignatov M.S., A.G. Bezgodov, O.I. Kuznetsova \& E.A. Ignatova 2018. On the genus Leskea (Leskeaceae, Bryophyta) in Russia. Arctoa 27:49-53.

${ }^{99}$ Ignatov, M.S., E.A. Ignatova, V.E. Fedosov, V.I. Zolotov, T. Koponen, I.V. Czernyadjeva, G.Ya. Doroshina, D.Ya. Tubanova \& N.E. Bell 2018. Moss flora of Russia. Vol. 4. Bartramiales - Aulacomniales. Moscow, 536 pp. (in Russian and English). [Игнатов M.С., Игнатова Е.A., Федосов В.Э., Золотов В.И., Копонен Т., Чернядьева И.В., Аорошина Г.Я., Тубанова А.Я., Бем Н.Э. 2018. ФАора мхов России. Том 4. Bartramiales - Aulacomniales. Москва: КМК. 543 с.].

${ }^{100}$ Ignatova, E.A. 2009. The genus Anoectangium (Pottiaceae, Bryophyta) in Russia. Arctoa 18:167-176.

${ }^{101}$ Ignatova, E.A. \& V.E. Fedosov 2008. Dicranum with fragile leaves in Russia. Arctoa 17:63-83.

${ }^{102}$ Ignatova, E.A. \& M.S. Ignatov 2011. The genus Thamnobryum (Neckeraceae, Bryophyta) in Russia. Arctoa 20:137-151.

${ }^{103}$ Ignatova E.A. \& J. Muñoz 2004. The genus Grimmia Hedw. (Grimmiaceae, Musci) in Russia. Arctoa 13:101-182.

${ }^{104}$ Ignatova E.A. \& O.Yu. Pisarenko 2013. New moss records from Sakhalin Province. 3. Sakhalin. In: New bryophyte records. 2. (E.V. Sofronova, ed.) Arctoa 22:258.

${ }^{105}$ Ignatova, E.A., O.I. Kuznetsova, H. Köckinger \& R. Hastings 2008. A preliminary study of Coscinodon in Eurasia (Grimmiaceae, Musci) in Eurasia based on morphology and DNA sequence data. Arctoa 17:1-18.

${ }^{106}$ Ignatova, E.A., M.S. Ignatov \& V.Ya.Cherdantseva 2009. The genus Neckera (Neckeraceae, Bryophyta) in the Russian Far East. Arctoa 18:177-188.

${ }^{107}$ Ignatova, E.A., H.H. Blom, D.V. Goryunov \& I.A. Milyutina 2010. On the genus Schistidium (Grimmiaceae, Musci) in Russia. Arctoa 19:195-233.

${ }^{108}$ Ignatova, E.A., M.S. Ignatov \& I.A. Milyutina 2010. A revision of the genus Lindbergia in Russia. Arctoa 19:97-116.

${ }^{109}$ Ignatova, E.A., V.Ya. Cherdantseva, O.V. Ivanov, I.V. Kostomarova \& M.S. Ignatov 2013. A preliminary list of mosses of the Botchinsky State Nature Reserve (Russian Far East). Arctoa 22:207-216.

${ }^{110}$ Ignatova, E.A., L.E. Kurbatova, O.I. Kuznetsova, O.V. Ivanov, J.R. Shevock, B. Carter \& M.S. Ignatov 2015. The genus Scouleria (Bryophyta) in Russia revisited. Arctoa 24:47-66.

${ }^{111}$ Ignatova, E.A., D.Ya. Tubanova, O.D. Tumurova, D.V. Goryunov \& O.I. Kuznetsova 2015. When the plant size matters: a new semi-cryptic species of Dicranum from Russia. Arctoa 24(2):471-488.

${ }^{112}$ Ignatova, E.A., H.H. Blom, O.I. Kuznetsova 2016. Schistidium austrosibiricum sp. nov. and S. scabripilum sp. nov. (Grimmiaceae, Bryophyta) - two closely related species from Asian Russia. Arctoa 25(1):107-115.

${ }^{113}$ Ignatova, E.A., M.S. Ignatov, T.I. Koroteeva \& D.Ya.Tubanova 2016. New moss records from Sakhalin Province. 7. The Kuril Islands. In: New bryophyte records. 6. (E.V. Sofronova, ed.). Arctoa 25:221-222.

${ }^{114}$ Ignatova E.A., O.I. Kuznetsova, V.E. Fedosov \& M.S. Ignatov 2016. On the genus Hedwigia (Hedwigiaceae, Bryophyta) in Russia. Arctoa 25(2):241-277.

${ }^{114 a}$ Ignatova, E.A., O.I. Kuznetsova \& M.S. Ignatov. 2017. Further comments on the genus Hedwigia (Hedwigiaceae, Bryophyta). Arctoa 26(2):132-143. 
${ }^{115}$ Ignatova E.A., O.I. Kuznetsova, I. A. Milyutina, V.E. Fedosov \& M.S. Ignatov 2017. On the genus Fabronia (Fabroniaceae, Bryophyta) in Russia. Arctoa 26 (1):11-34.

${ }^{116}$ Ivanov, O.V., M.A. Kolesnikova, T.V. Akatova, O.M. Afonina, E.Z. Baisheva, A.G. Bezgodov, O.A. Belkina, I.V. Czernyadjeva, S.V. Dudov, V.E. Fedosov, E.I. Ivanova, E.A. Ignatova, M.N. Kozhin, E.D. Lapshina, A.A. Notov, O.Yu. Pisarenko, N.N. Popova, A.N. Savchenko, V.V. Teleganova, D.Ya. Ukrainskaya \& M.S. Ignatov 2017. The database of the moss flora of Russia. Arctoa 26(1):1-10.

${ }^{117}$ Ivanova E.I. \& M.S. Ignatov 2007. The genus Lyellia R.Br (Polytrichaceae, Bryophyta) in Russia. Arctoa 16:169-174.

${ }^{118}$ Ivanova, E.I., M.S. Ignatov, I.A. Milutina \& V.K. Bobrova 2005. On the morphological and molecular differences between Oligotrichum bercinicum and O. falcatum (Polytrichaceae, Bryophyta). Arctoa 14:1-12.

${ }^{119}$ Ivanova, E.I., N.E. Bell, O.I. Kuznetsova, H. Lee, M. Park \& M.S. Ignatov 2014. The genus Polytrichastrum (Polytrichaceae) in Russia. Arctoa 23:164-184.

${ }^{120}$ Ivanova, E.I., N.E. Bell, O.I. Kuznetsova, E.A. Ignatova \& M.S. Ignatov 2015. The genus Polytrichum sect. Aporotheca (Polytrichaceae) in Russia. Arctoa 24:67-78.

${ }^{121}$ Kolesnikov, B.P. 1938. Toward the moss flora of central Sikhote-Alin. In: Proceedings of the Sikhote-Alin state reserve. Vol. 1, pp. 209-227 (in Russian). [Колесников Б.П. 1938. Материалы к флоре мхов срелнего Сихотэ-Алиня // Труды Сихотэ-Алинского государственного заповеАника. Вып. 1. С. 209-227].

${ }^{122}$ Koponen, T. \& O. Afonina. 1992. Miscellaneous notes on Mniaceae (Bryophyta), XV. Bryobrothera 1:245-250.

${ }^{123}$ Koponen, T., I. Järvinen \& P. Isoviita 1978. Bryophytes from the Soviet Far East, mainly the Khabarovsk territory. Annales Botanici Fennici 15:107-121.

${ }^{124}$ Koponen, T., E.A. Ignatova, O.I. Kuznetsova \& M.S. Ignatov 2012. The genus Philonotis (Bartramiaceae, Musci) in Russia. Arctoa. 21:21-62.

${ }^{125}$ Koroteeva T.I., A.V. Fedorova \& M.S. Ignatov 2016. Cyrto-hypnum versicolor (Thuidiaceae, Bryophyta) in Russia. Arctoa 25:80-88.

${ }^{126}$ Kuptsova, V.L., M.S. Ignatov, E.N. Zakharchenko, A.V. Ostroukhov \& E.M. Klimina 2018. On the record of Sasaokaea aomoriensis (Leskeaceae, Bryophyta) in Bolonsky Reserve, Russian Far East. Botanicheskii Zhurnal 103(2): 249-255 (in Russian with English summary). ККупцова B.А., Игнатов M.C., Захарченко Е.Н., Остроухов А.В., КАимина E.M. 2018. Находка Sasaokaea aomoriensis (Leskeaceae, Bryophyta) в заповеднике Болоньский (Российский Аальний Восток) // Ботанический журнал. T. 103, № 2. C. 249-255].

${ }^{127}$ Kuzmina, E.Yu., T.I. Koroteeva, E.V. Sofronova \& R.R. Sofronov 2014. New moss records in Sakhalin Province. 4. In: New bryophyte records. 4. (E.V. Sofronova, ed.) Arctoa 23:235. (in Russian) [Кузьмина Е.Ю., Коротеева Т.И., Софронова Е.В., Софронов Р.Р. 2014. Новые находки мхов в Сахалинской области. 4 // Новые бриомогические находкию 4 / под реА. Е.В. Софроновой. Arctoa. Вып. 23. С. 235].

${ }^{128}$ Kuzmina, E.Yu., T.I. Koroteeva, E.A. Ignatova \& M.S. Ignatov 2016. New moss records from Sakhalin Province. 7. The Kuril Islands. In: New bryophyte records. 7 (E.V. Sofronova, ed.). Arctoa 25:450 (in Russian). [Кузьмина Е.Ю., Коротеева Т.И., Игнатова Е.А., Игнатов М.С. 2016. Новые находки мхов в Сахалинской области 7.
Курильские острова // Новые находки мохообразных. 7. / под реА. Е.В. Софроновой. Arctoa. Вып. 25. C. 450 .

${ }^{128 a}$ Lang, A.S. \& M. Stech 2014. What's in a name? Disentangling the Dicranum scoparium species complex (Dicranaceae, Bryophyta). Systematic Botany 39(2):369-379.

${ }^{129}$ Lazarenko, A.S. 1936. Brief handbook of mosses of Far East. Vladivostok, Dalnevostochnoe Otdelenie Akademii Nauk SSSR. 101 pp. (in Russian). [Аазаренко A.C. 1936. Краткий определитель миственных мхов Аальнего Востока. ВАадивосток, АВО АН СССР. 101 с.].

${ }^{130}$ Lazarenko, A.S. 1940. Leafy mosses of the Soviet Far East. I (Acrocarpae: Andreaeales-Schistostegiales). Botanicheskii Zhurnal Akademii Nauk Ukrainskoi RSR 1(3-4):239-243 (in Ukrainian). [Аазаренко А.С. 1940. Аистяні мохи Радянського Аалекого Сходу I. ВерхопліАні мохи (Acrocarpae: Andreaeales-Schistostegiales) // Ботанический журнал АН УРСР. Т. 1, № 3-4. С. 239-243].

${ }^{131}$ Lazarenko, A.S. 1941a. Leafy mosses of the Soviet Far East. II. Botanicheskii Zhurnal Akademii Nauk Ukrainskoi RSR 2(1):51-95 (in Ukrainian). [Аазаренко A.C. 1941 a. Аистяні мохи Рацянського Аалекого Сходу II. Acrocarpae: Orthotrichales-Eubryales; Pleurocarpae: Isobryales-Hypnobryales (Theliaceae-Leskeaceae) // Ботанический журнал АН УРСР. Т 2, № 1. С.51-95].

${ }^{132}$ Lazarenko, A.S. 1941b. Leafy mosses of the Soviet Far East. III. Thuidiaceae-Brachytheciaceae. Botanicheskii Zhurnal Akademii Nauk Ukrainskoi RSR 2(2):271-308 (in Ukrainian). [Аазаренко A.C. 1941b. Аистяні мохи РаАянського Аалекого Сходу III. Thuidiaceae-Brachytheciaceae // Ботанический журнал АН УРСР. Т. 2, № 2. C. 271-308].

${ }^{133}$ Lazarenko, A.S. 1945. Leafy mosses of the Soviet Far East. IV. Entodontaceae - Hylocomiaceae. Botanicheskii Zhurnal Akademii Nank Ukrainskoi RSR 2(3-4):185-216 (in Ukrainian). [Аазаренко А.С. 1945. Аистяні мохи РаАянського Аалекого Сходу IV. Entodontaceae-Hylocomiaceae // Ботанический журнал АН УРСР. Т. 2, № 3-4. C.185-216].

${ }^{134}$ Lazarenko, A.S. 1946. Bryological notes. Botanicheskii Zhurnal Akademii Nauk Ukrainskoi RSR 3(3-4):61-63 (in Ukrainian). [Аазаренко А. C. 1946. Бріологічні нотатки // Ботанический журнал АН УРСР Т. 3 № 3-4. С. 61-63].

${ }^{135}$ Lindberg, S.O. 1872. Contributio ad Floram Cryptogamam Asiae Boreali-Orientalis. Acta Societatis Scientiarum Fennicae 10:221-280.

${ }^{136}$ Magill, R.E. \& V.Ya. Cherdantseva 1995. Meteorium (Musci, Meteoriaceae) in the Russian Far East. Fragmenta Floristica et Geobotanica 40(1):223-227.

${ }^{137}$ Maksimov, A.I. 2007. Sphagnum imbricatum complex (Sphagnaceae, Bryophyta) in Russia. Arctoa 16:25-34.

${ }^{138}$ Maksimov A.I. \& E.A. Ignatova 2008. Sphagnum alaskense (Sphagnaceae, Bryophyta), a new species for Russia. Arctoa 17:109-112.

${ }^{139}$ Maksimov, A.I. \& E.S. Koryagina 2013. New moss records in Amur Province. 1. Arctoa 22:256-257 (in Russian). [Максимов А.И., Корягина Е.С. 2013. Новые находки мхов в Амурской области. 1 // Новые находки мохообразных. 2 / под ред. Е.В. Софроновой. Arctoa. Vol. 22. P. 256-257].

${ }^{140}$ Maksimov, A.I., V.E. Fedosov \& E.A. Ignatova 2016. Sphagnum beringiense (Sphagnaceae, Bryophyta) in Russia. 
Arctoa 25:102-106.

${ }^{141}$ Nyushko T.I., E.A. Ignatova \& M.S. Ignatov 2008. Contribution to moss flora of the Kuril Islands (Russian Far East). Arctoa 17:165-168.

${ }^{142}$ Otnyukova, T.N. 2001. Notes on Dicranum (Dicranaceae, Musci) in Russia. 1. Dicranum nipponense found in Far East. Arctoa 10:157-160.

${ }^{143}$ Otnyukova, T.N. \& R. Ochyra 2003. On Dicranum dispersum Engelmark and Dicranum orientale Otnyukova. Arctoa 12:115-116.

${ }^{144}$ Pisarenko, O.Yu. 2016. New moss records from Primorsky Territory. 3. In: New bryophyte records. 7 (ed. E.V. Sofronova). Arctoa 25:448.

${ }^{145}$ Pisarenko O.Yu. \& V.E. Fedosov 2016. New moss records from Khabarovsk Territory. 3. In: New bryophyte records. 7 (E.V. Sofronova, ed.). Arctoa 25:446-447.

${ }^{146}$ Pisarenko, O.Yu. \& V.E. Fedosov 2017. New moss records from Primorsky Territory. 4. In: New bryophyte records. 8 (E.V. Sofronova, ed.). Arctoa 26:121-122.

146a Pisarenko, O.Yu., V.E. Fedosov \& V.A. Bakalin 2017. Mosses of the Russian Far East Exiccatae. Fasc. III (№№ 71-105). Vladivostok, 14 pp.

${ }^{147}$ Podperra J. 1929. Musci insulae Rossicae prope Vladivostok. Publications of the Faculty of Science of the University of Masaryk. 116:3-40

${ }^{148}$ Podpĕra, J. 1954. Conspectus Muscorum Europaeorum. Prague, 697 pp.

${ }^{149}$ Regel, E. 1861. Tentamen florae Ussuriensis. St-Petersburg, 38 pp.

${ }^{150}$ Sakurai, K. 1935. Beabachtungen über japanische Moosflora. VII. Botanical Magazine, Tokyo 49(579):126-144.

${ }^{151}$ Schmidt, F.B. 1869. Reisen in Amurland und auf Insel Sachalin, im Auftrage der Kaiserlichen. Russischen Geographischen Gesselschaft ausgefürcht. Mémoires de l'Académie impériale des sciences de St. Pétersbourg, ser. 7. 12(2): 74-75.

${ }^{152}$ Sofronova, E.V. (ed.), O.M. Afonina, S.M. Aznabaeva, E.Z. Baisheva, A.N. Bersanova, A.G. Bezgodov, E.A. Borovichev, M.A. Boychuk, E.V. Chemeris, G.Ya. Doroshina, M.V. Dulin, A.P. Dyachenko, V.E. Fedosov, I.V. Filippov, E.V. Garin, O.G. Grishutkin, M.S. Ignatov, E.A. Ignatova, E.I. Ivanova, M.A. Kolesnikova, T.I. Koroteeva, G.M. Kukurichkin, S.A. Kutenkov, E.Yu. Kuzmina, E.D. Lapshina, O.V. Lavrinenko, A.I. Maksimov, K.O. Pechenkina, D.A. Philippov, O.Yu. Pisarenko, N.N. Popova, Yu.M. Sergeeva, E.A. Shchipanova, G.S. Taran, V.V. Teleganova \& D.A. Zakharchenko 2018. New Bryophyte Records. 10. Arctoa 27:60-86.

${ }^{153}$ Sugawara S. 1956. Bryophyta sachalinenses.Shyokubutsuken Kyukai, Hakodate, 55 pp. (In Japanese).
${ }^{154}$ Tubanova, D.Ya., \& E.A. Ignatova 2011. A new species of Dicranum from Asiatic Russia. Arctoa 20:183-190.

${ }^{155}$ Tubanova D.Ya., D.V. Goryunov, E.A. Ignatova \& M.S. Ignatov 2010. On the taxonomy of Dicranum acutifolium and D. fuscescens complexes (Dicranaceae, Bryophyta) in Russia. Arctoa 19:151-164.

${ }^{156}$ Tubanova, D.Ya., L.Kh. Dorzhieva \& O.D. Tumurova 2016. New moss records in Sakhalin Procince 6. Sakhalin. In: New bryophyte records. 7 (E.V. Sofronova, ed.). Arctoa 25:450. (in Russian). [Тубанова А.Я., Аоржиева А.X, Тумурова О.А. Новые находки мхов в Сахалинской области 6. Остров Сахалин. // Новые находки мохообразных. 7 / под ред. Е.В. Софроновой. Arctoa. Vol. 25. P. 450].

${ }^{157}$ Tubanova, D.Ya, O.D. Tumurova \& E.A. Ignatova 2016. On Dicranum elongatum and D. groenlandicum in Russia. Arctoa 25(2):285-300.

${ }^{158}$ Tubanova, D.Ya., O.D. Tumurova \& E.A. Ignatova 2017. The genus Mielichhoferia (Mielichhoferiaceae, Bryophyta) in Russia. Arctoa 26(2):166-180.

${ }^{159}$ Tubanova, D.Ya., V.E. Fedosov \& O.D. Dugarova 2018. Dicranum ignatovii sp. nova (Dicranaceae, Bryophyta) from the Far East. Philippine Journal of Systematic Biology 12(1): $37-44$.

${ }^{160}$ Ukrainskaya, G.Y. 1996. On similarity and differences of Plagiothecium piliferum (Sw.) B.S.G. and Isopterygiopsis muelleriana (Schimp.) Iwats. Novosti Sist. Nizsh. Rast. 31: 185-191 (in Russian). ГУкраинская Г.Я. 1996. О сходстве и различиях Plagiothecium piliferum (Sw.) B.S.G. и Isopterygiopsis muelleriana (Schimp.) Iwats. // Новости систематики низших растений. Т. 31. С. 185-191].

${ }^{161}$ Warnstorf, C. 1913. Zur Bryo-Geographie des Russischen Reiches [Pt. 1]. Hedwigia 53: 184-320.

${ }^{162}$ Warnstorf, C. 1914. Zur Bryo-Geographie des Russischen Reiches [Pt. 2]. Hedwigia 54: 22-112.

${ }^{163}$ Yakovchenko, L.S., I.A. Galanina, V.A. Bakalin \& E.V. Malashkina 2013. Bryophytes and lichens of old-growth forests in lower Amur River area. Komarovskie Chteniya 60:8-68 (in Russian with English summary). [Яковченко А.С., Галанина И.А., Бака^ин В.А., Махашкина Е.В. 2013. Мохообразные и мишайники малонарушенных месных сообществ в нижнем Приамурье (Российский АаАьний Восток) // Комаровские чтения. Т. 60. С. 8-68].

${ }^{164}$ Zolotov, V.I. 2011. The genus Bryum Hedw. In: Moss flora of Russia. On-line version, $51 \mathrm{pp}$ (in Russian). [Золотов В.И. 2011. РоА Bryum Hedw. - Бриум / ФАора мхов России. Эмектронная публикация. 51 с.]. http:// arctoa.ru/Flora/taxonomy-ru/Bryum-text-russian.pdf). версия 1.V.2011 